Seunghoe Roh

\title{
Assessment of indoor localization algorithm performance under realistic conditions
}

School of Electrical Engineering

Thesis submitted for examination for the degree of Master of Science in Technology.

Espoo September 7, 2012

Thesis supervisor:

Prof. Jorma Skyttä

Thesis instructor:

M.Sc. Juha Forsten 
Author: Seunghoe Roh

Title: Assessment of indoor localization algorithm performance under realistic conditions

Date: September 7, $2012 \quad$ Language: English $\quad$ Number of pages: $11+64$

Department of signal processing and acoustics

Professorship: Digital signal processing

Code: S-88

Supervisor: Prof. Jorma Skyttä

Instructor: M.Sc. Juha Forsten

Wireless sensor networks localization is one of most vibrant research field since it has more advantageous than a global positioning system (GPS) in aspect of cost and indoor usability. In wireless sensor networks (WSN), predetermined location of a sensor can sometimes be meaningless because a sensor can be deployed in such an area where is hardly reachable. Therefore, a sensor must be able to localize itself and provides its location information to other sensor nodes.

Non-cooperative localization, also called traditional localization, like local positioning system (LPS) has been done by communications only between anchor nodes and an agent node. However, a sensor requires to have a sensor that has unlimited power transmission capability, which seems unrealistic. Furthermore, accuracy is also limited due to communication only between an anchor node and an agent node. To deal with these problems, cooperative localization technique has been suggested and studied. Cooperative localization is the technique including not only measurement between anchor nodes and an agent node but also the measurement between agent nodes for location estimation so as to increase positioning accuracy and robustness.

Among various sensory modalities (acoustic, seismic, infrared and so on) the modality of measurement made in wireless sensor networks throughout this thesis is radio frequency $(\mathrm{RF})$. Simulation results of both non-cooperative and cooperative localization accuracy will be observed in order to prove that cooperative localization outperforms non-cooperative localization. In addition, simulated data is compared to data which is actually measured in indoor environment to give the idea how reliable the simulation is in indoor.

Keywords: Wireless sensor networks (WSN), anchor node, agent node, traditional localization, cooperative localization 


\section{Acknowledgement}

First of all, I want to thank my supervisor Professor Jorma Skyttä and instructor Juha Forsten and for directing my thesis. I also like to express my gratitude to Dr. Andreas Ritcher, former supervisor, who suggested the thesis topic as well as provided overall structure of my thesis. Without all these people it would not be possible for me to complete this master's thesis.

I truly appreciate my friends living in all over the world. First of all, my friends in Otaniemi, Kyunghyun Cho, Byungjin Cho, Wonjae Kim, and Henok Adane helped me a lot practically and emotionally. Their encouragement boosted the progress of my thesis, which did not seem to be over forever. And second, my Indonesian sister, Angela Lestari, supported my work all the way from United States through phone call and e-mail. Her advice on my thesis is truly valuable and grateful. And my Chinese friend, Prof. Ying Zhou, made a huge contribution for figuring out how the structure of my work should be. I thank Jussi Salmi and Marió Costa for the effort made for thesis revision.

Last but foremost my family's everlasting love and support toward me is unforgettable and plays the key role for me in completing this thesis. Their contribution and sacrifice all along my study period is beyond description.

Otaniemi, September 7, 2012

Seunghoe Roh 


\section{Contents}

$\begin{array}{ll}\text { Acknowledgement } & \text { iii }\end{array}$

Contents

List of abbreviations vi vi

List of symbols vii

List of Figures $\quad$ x

List of Tables $\quad$ xi

1 Introduction $\quad 1$

1.1 Structure of wireless sensor network . . . . . . . . . . . . . . . 1

1.2 Motivation and objective . . . . . . . . . . . . . . . . . 3

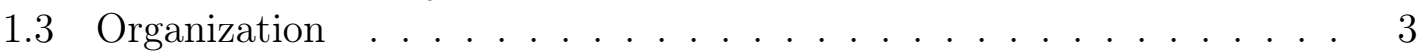

2 Location estimation in wireless sensor networks 5

2.1 Distance measurement using radio frequency . . . . . . . . . . . 5

2.1.1 Received signal strength . . . . . . . . . . . . 5

2.1 .2 Time of arrival . . . . . . . . . . . . . . 7

2.2 Position estimation algorithm . . . . . . . . . . . . . . . . . 8

2.2.1 Least squares estimation . . . . . . . . . . . . . . . . . . . . . . . . . . . 9

2.2 .2 Joint map estimation . . . . . . . . . . . . . . . . . . . . 12

2.2.3 Conjugate descent . . . . . . . . . . . . . . . . . . 14

2.3 Cramér-Rao lower bound . . . . . . . . . . . . . . . . . . . 20

$\begin{array}{lll}3 & \text { Setup and analysis for the simulation of various grid patterns } & 22\end{array}$

3.1 Set up and explanation of simulation . . . . . . . . . . . . 22

3.1.1 Scenario and process . . . . . . . . . . . . . . 23

3.1.2 Set-up for received signal strength . . . . . . . . . . . . . . . 26

3.1.3 Set-up for time of arrival . . . . . . . . . . . . . . . 28

3.2 Analysis of simulation of RSS with varying sensor density . . . . . . . 29

3.3 Analysis of simulation of RSS with varying transmission range . . . . 33

3.4 Analysis of simulation of TOA with varying the sensor density . . . . 37

3.5 Analysis of simulation of TOA with varying transmission range . . . . 41 
4 Analysis of measured data and comparison to simulated data 46

4.1 Basic information about measurement . . . . . . . . . . . . . 46

4.2 Comparison for RSS based localization . . . . . . . . . . . . . . . . . . . . 47

4.2.1 Least squares based localization . . . . . . . . . . . . . . . . 49

4.2 .2 Joint map estimation . . . . . . . . . . . . . . . . . . 51

4.2.3 Comparison of all algorithms . . . . . . . . . . . . . . . 52

4.3 Comparison for TOA based localization . . . . . . . . . . . . . 53

4.3.1 Least squares based method . . . . . . . . . . . . . . . 54

4.3 .2 Joint map estimation . . . . . . . . . . . . . . . . . . . . 56 56

4.3.3 Comparison of all algorithms . . . . . . . . . . . 57

$\begin{array}{llr}5 & \text { Conclusions } & 58\end{array}$

$\begin{array}{lr}\text { Bibliography } & 60\end{array}$ 


\section{Abbreviations}

$\begin{array}{ll}\text { WSN } & \text { Wireless sensor network } \\ \text { RF } & \text { Radio frequency } \\ \text { GPS } & \text { Global positioning system } \\ \text { LPS } & \text { Local positioning system } \\ \text { MCU } & \text { Micro-controller } \\ \text { IP } & \text { Internet Protocol } \\ \text { RSS } & \text { Received signal strength } \\ \text { TOA } & \text { Time of arrival } \\ \text { SNR } & \text { Signal-to-noise ratio } \\ \text { OS } & \text { Offset time } \\ \text { CRLB } & \text { Cramér-Rao lower bound } \\ \text { LSE } & \text { Least squres estimation } \\ \text { LOS } & \text { Line-of-sight } \\ \text { DS-SS } & \text { Direct-sequence spread-spectrum } \\ \text { UWB } & \text { Ultra wide band } \\ \text { PDF } & \text { Probability density function } \\ \text { RMSE } & \text { Root mean squared error }\end{array}$




\section{List of Symbols}

$d \quad$ True distance between sensor nodes

$\tilde{d} \quad$ Distance estimated in noisy environment

$\bar{P}(d) \quad$ Average power transmitted between sensor nodes

$n_{p} \quad$ Path loss coefficient

$d_{0} \quad$ Reference distance

$\operatorname{cov}(\hat{\theta}) \quad$ Covariance matrix of unbiased estimator

$P_{0} \quad$ Received power at reference distance $d_{0}$

$\mathbf{F}_{\theta} \quad$ Fisher information matrix at the parameter $\theta$

$\mathcal{N}\left(0, \sigma^{2}\right)$ Gaussian distribution with zero mean and standard deviation $\sigma$

$\sigma_{\mathbf{d B}}, \sigma_{\mathbf{d B}}^{2} \quad$ Standard deviation, Variance in $\mathrm{dB}$

$\bar{P}\left(d_{i, j}\right) \quad$ Average received power between receiver and transmitter

$p \quad$ Probability density

$\nabla_{\theta} \quad$ Derivative with respect to $\theta$

$f(\mathbf{X} \mid \theta) \quad$ Conditional probability density function of random variables $\mathbf{X}$ given $\theta$

$T_{\mathrm{ft}}, T_{\mathrm{fr}} \quad$ Time that forward signal is transmitted and received respectively

$T_{\mathrm{rt}}, T_{\mathrm{rr}} \quad$ Time that reverse signal is transmitted and received respectively

$P_{r} \quad$ Received power at receiver in $\mathrm{dBm}$

$P_{t} \quad$ Output power transmitted in $\mathrm{dBm}$

$P_{L} \quad$ The loss of power propagating in $\mathrm{dB}$

$\mathbf{A}_{\mathrm{col}} \quad$ Column vector of matrix $A$

$J \quad$ Fisher information

$\hat{\theta} \quad$ Unbiased estimator of $\theta$

$f \quad$ Radio frequency in $\mathrm{MHz}$

$\mathbf{F}_{\mathrm{xx}} \quad$ Partial Fisher information matrix derived using only $x$ coordinate of unknown node to be estimated

$\mathbf{F}_{\text {yy }} \quad$ Partial Fisher information matrix derived using only $y$ coordinate of unknown node to be estimated

$\mathbf{F}_{\mathrm{xy}} \quad$ Partial Fisher information matrix derived using both $x$ and $y$ coordinates of unknown node to be estimated

$H(k) \quad$ Set of sensor that sensor $k$ makes measurement with

$I_{H(k)} \quad$ Indicator function with respect to matrix $H(k)$

$\gamma \quad$ Channel constant in Cramér-Rao lower bound matrix

$\mathbb{R}^{2 n \times 2 n} \quad 2 n \times 2 n$ real number parameter matrix 
$A_{\mathrm{xi}}, A_{\mathrm{yi}} \quad x, y$ coordinates of $i$ th anchor node

$U_{\mathrm{xi}}, U_{\mathrm{yi}} \quad x, y$ coordinates of $i$ th unknown node

$c \quad$ Cost function

$N\left(0, \sigma^{2}\right) \quad$ Guassian distribution with zero mean

$I_{H(k)}(l) \quad$ Indicator function, where if $l \in H(k)$, it is 1 otherwise 0

$U_{r m s} \quad$ Average root mean squared error of unknown nodes

$U_{\mathrm{CRB}} \quad$ Average root mean squared Cramér-Rao lower bound

$v_{p} \quad$ Propagation velocity

$\mu_{t} \quad$ Mean of time delay error

$\tau \quad$ Time of arrival 


\section{List of Figures}

1.1 Wireless Sensor network . . . . . . . . . . . . . . . . . 1

1.2 Block diagram of a sensor node . . . . . . . . . . . . . . 2

2.1 General radio signal transmitting system . . . . . . . . . . . 5

2.2 Time of arrival . . . . . . . . . . . . . . . . 7

2.3 The closest point to the vector space . . . . . . . . . . . . . 9

2.4 The orthogonal decomposition . . . . . . . . . . . . . . . . . 10

2.5 Fitting a line based on data . . . . . . . . . . . . . . . . . . 11

2.6 Comparison of direction vectors . . . . . . . . . . . . . . 19

3.1 Anchor nodes deployed in three different grid patterns . . . . . . . . . 23

3.2 The area where anchor nodes and agent nodes are deployed . . . . . . 24

3.3 Non-cooperative least squares estimation based on RSS . . . . . . . . 30

3.4 Joint map estimation based on RSS . . . . . . . . . . . . . . . . . . . 31

3.5 Scaled conjugate gradient based on RSS . . . . . . . . . . . . . 32

3.6 All RSS-based localization results with varying density . . . . . . . . 32

3.7 Linear least Squares estimation based on RSS . . . . . . . . . . . . . 34

3.8 Joint map estimation based on RSS . . . . . . . . . . . . . . . . . 35

3.9 Scaled conjugate gradient based on RSS . . . . . . . . . . . . 36

3.10 All RSS-based localization results with varying transmission range . . 37

3.11 Linear least Squares estimation based on TOA . . . . . . . . . . . 38

3.12 Joint map estimation based on TOA . . . . . . . . . . . . . . . . . 39

3.13 Scaled conjugate gradient based on TOA . . . . . . . . . . . . . . 40

3.14 All TOA-based localization results with varying density . . . . . . . . 41

3.15 Linear least Squares estimation based on TOA . . . . . . . . . . . . . 42

3.16 Joint map estimation based on TOA . . . . . . . . . . . . . . 43

3.17 Scaled conjugate gradient based on TOA . . . . . . . . . . . . . . 44

3.18 All TOA-based localization results with varying transmission range . 45

4.1 The position of sensor nodes deployed at research lab . . . . . . . . . 46

4.2 RSS based least squares estimation with CRLB . . . . . . . . . . . 49

4.3 Comparison of PDF of noise for RSS . . . . . . . . . . . . . . . 50

4.4 Joint map estimation based on RSS data . . . . . . . . . . . . . . . 52

4.5 All algorithms with CRLB based on RSS data . . . . . . . . . . . 52

4.6 Distance vs Noise . . . . . . . . . . . . . . . . . . . . . 54

4.7 TOA based least squares estimation . . . . . . . . . . . . . 55 
4.8 Comparison of PDF of noise for TOA . . . . . . . . . . . . . . . . 55

4.9 Joint map estimation based on TOA data . . . . . . . . . . . . . 56

4.10 All algorithms with CRLB based on TOA data . . . . . . . . . . 57 


\section{List of Tables}

3.1 Parameter values for RSS based simulation . . . . . . . . . . . . . 26

3.2 Parameter values for TOA based simulation . . . . . . . . . . . 28

3.3 Parameter values for RSS with varying a sensor density . . . . . . . . 29

3.4 Parameter values for RSS with varying a transmission range . . . . . 33

3.5 Parameter values for TOA with varying a sensor density . . . . . . . 38

3.6 Parameter values for TOA with varying a transmission range . . . . . 42

4.1 The specification of a sensor node . . . . . . . . . . . . . . . . 47

4.2 The measurement data of RSS . . . . . . . . . . . . . . . . . . 48

4.3 RSS based localization set-up . . . . . . . . . . . . . . . . . . . . . . . . . . . . . . 58

4.4 The measurement data of TOA . . . . . . . . . . . . . . 53

4.5 TOA based localization set-up . . . . . . . . . . . . . . . 54 


\section{Chapter 1}

\section{Introduction}

\subsection{Structure of wireless sensor network}

An interest in wireless sensor networks has rapidly grown due to its potential use for countless applications including commercial and military applications like control, automation, environmental monitoring, medical application, agriculture and surveillance [1]-[3]. For instance, people engaged in agriculture would like to monitor soil and air, as well as plant and for people in animal care desire to observe behavior patterns and interactions between animal species using various sensors like acoustic, seismic, and radio frequency (RF) sensor. Due to remote communication, energy-efficient communication strategy is key issue for deploying wireless sensor networks [2].

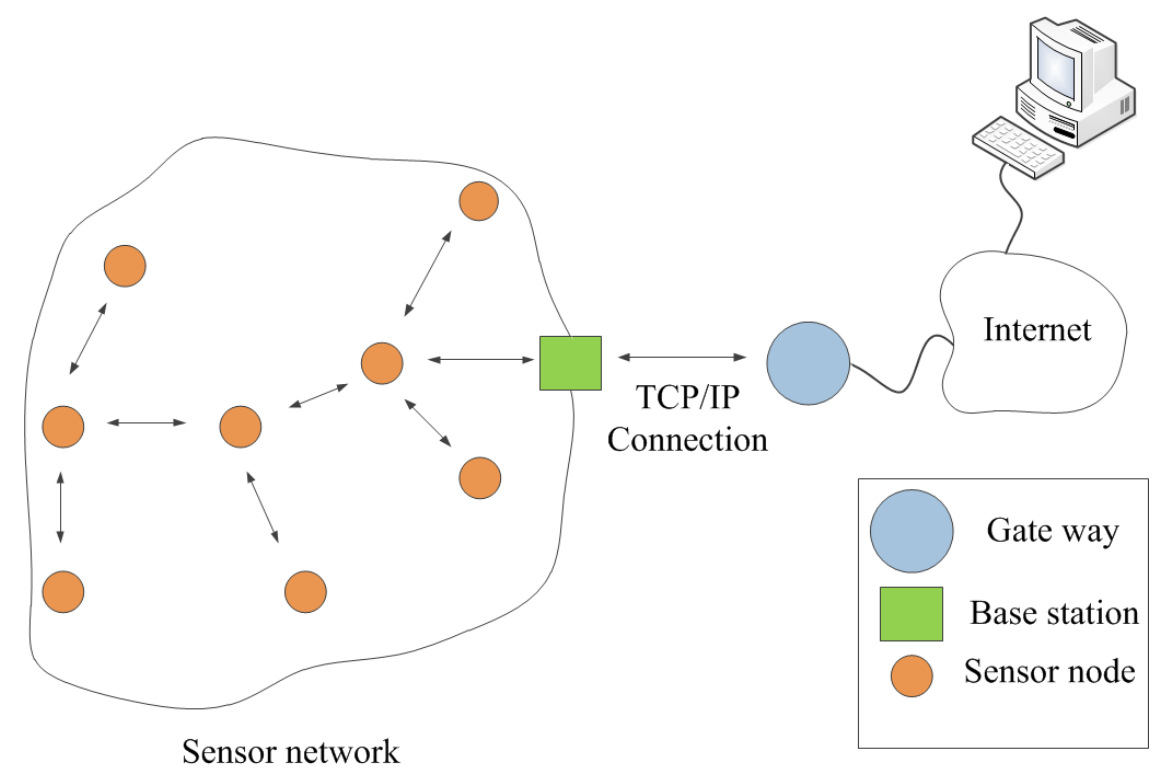

Figure 1.1: Wireless Sensor network

A wireless sensor network is comprised of a vast number of sensor nodes communicating to one another in ad-hoc manner. Query messages from users are 
distributed to sensor network through a gateway, and responses from sensor networks are gathered to base station, also referred to as sink node. A sensor gateway is used to route the sensor network to the Internet [4]. In addition, it also processes data assessment at each sensor and data storage service. The storage services are present between user and sensors, which is providing space for data. And also, data storage is added to the IP network to store sensor data from edge sensor networks and to support various user-initiated browsing and search functions [5]. A base station, a node where communication ends, connects the sensor network to another network to convey the data gained [6].

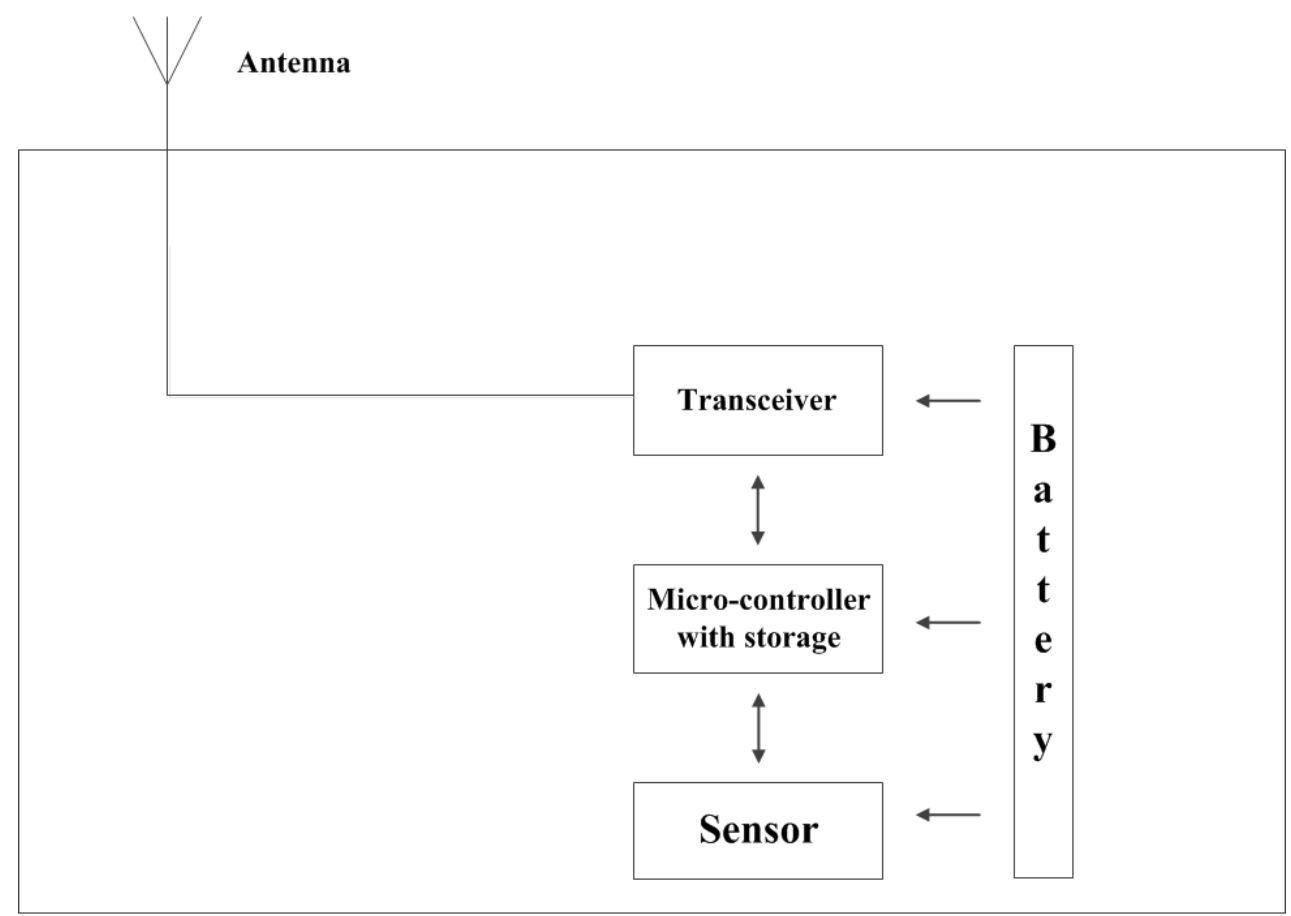

Figure 1.2: Block diagram of a sensor node

As shown in Figure 1.2 [7]a sensor node is usually comprised of a radio frequency $(\mathrm{RF})$ transceiver with an antenna, a micro-controller (MCU), and a battery. When it comes to sensor nodes, power consumption has always been the biggest concern since all sensor nodes are powered by battery. As transceiver (transmitterreceiver), the component makes it possible to realize wireless communication between sensor nodes, typically consumes most power among components in a sensor node, it is the most core component of a wireless sensor node among components of a sensor node [8]. Therefore, the technology enabling communication by low power and ideal balance between data rate and power consumption are needed [9]. And a micro-controller (MCU), the other component, is used to process the data measured from sensors and transmits the data to transceiver [10], [11]. A node can either be deployed at pre-fixed or random location to sense physical or environmental change like motion, temperature, sound, vibration, image and so on [12].

Sensor nodes in wireless sensor network (WSN) can be sorted to two groups - an anchor node and an agent node. An anchor node means a node whose loca- 
tion is initially known whereas an unknown node refers to a node whose location is priori unknown. It is often called as an agent node as well. Traditional localization method, non-cooperative localization method, utilizes distance informations between anchor nodes and an unknown node only. On the other hand, cooperative localization method utilizes distance informations even between unknown nodes to improve accuracy and to overcome the limitation of power transmission range.

\subsection{Motivation and objective}

Due to energy limitation, the way to enable low-power wireless communication has been studied and suggested in the wireless network field. The research interest of localization technique in wireless sensor networks (WSN) localization has been increased due to its various applications such as animal species monitoring, and target tracking [13]. Accurate and low-power sensor localization is crucial and challenging for the deployment of wireless sensor networks. There are decent amount of research papers suggesting way of realizing cooperative localization in order to improve accuracy and power efficiency.

Therefore, this paper conducts non-cooperative and cooperative localization and compares accuracy. To briefly explain about first part of work, using received signal strength (RSS) and time of arrival (TOA) non-cooperative and cooperative localization are simulated under scenario where anchor nodes are deployed in three different patterns. The accuracy of both non-cooperative and cooperative method are observed as a sensor density is varied with fixed transmission range first. After that, the accuracy of localization algorithm are again calculated as a transmission is varied range with fixed sensor density. Through the simulations, both non-cooperative and cooperative localization algorithms are observed in aspect of accuracy and variance. In addition, it is possible to observe which pattern of anchor nodes deployed shows the best accuracy.

Another work is to verify how similar simulated data is to measured data. Using the parameters of RSS and TOA data obtained from some researcher's experiment, RSS and TOA data are generated under particular noise distribution. By comparing simulated data based localization to measured data based one, one can learn how reliable simulated data is.

\subsection{Organization}

The contents of this thesis are organized as follows. Chapter 2 introduces basic concepts of necessary localization-related knowledge including a several measurements, which are used to estimate a distance and location estimation algorithms. In Chapter 3, simulation results on three different anchor node grid with localization algorithms mentioned in Chapter 2 are compared to one another in order to find out best grid formation and algorithms. 
Chapter 4 analyzes data measured in an experiment and compares it to simulated data for the purpose of observing how viable simulated data is. Finally, Chapter 5 concludes all the results of simulations conducted throughout this thesis. 


\section{Chapter 2}

\section{Location estimation in wireless sensor networks}

This chapter provides fundamental knowledge regarding a location estimation in the following order. First, location measurements such as received signal strength (RSS) and time of arrival (TOA) are introduced with corresponding noise channel applicable to the measurement. Then, both non-cooperative and cooperative localization methods are explained. Last, Cramér-Rao lower bound, lower bound on the possible covariance by any existing unbiased location estimator, is illustrated with formula.

\subsection{Distance measurement using radio frequency}

\subsubsection{Received signal strength}

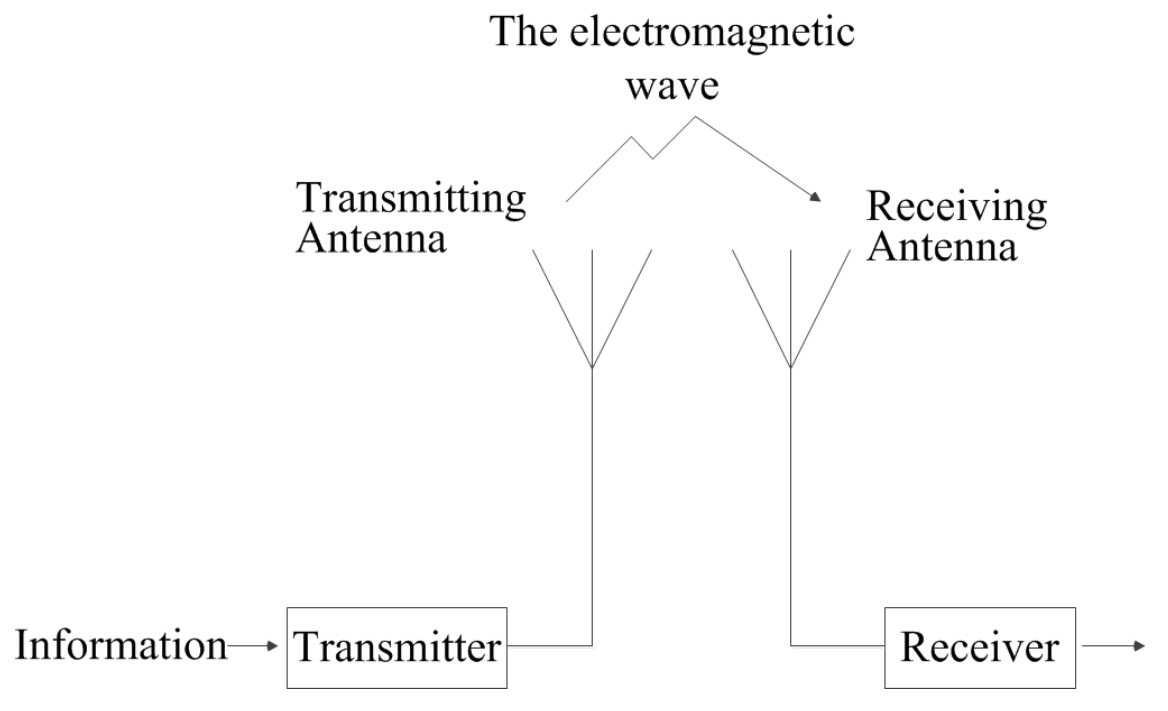

Figure 2.1: General radio signal transmitting system

A general and a simple radio system consists of antennas, transmitter, and receiver as shown in Figure 2.1. To briefly explain the radio signal processing, 
the signal including information is first modulated through transmitter. Then, the modulated radio signal is converted into an electromagnetic wave by transmitting antenna. After the electromagnetic wave travels through medium, free space, it is captured by receiving antenna which converts it to electronic signal. And finally, the converted signal is demodulated back to original signal involving information [14].

There are major factors causing the power loss of the radio frequency (RF) signal, which is called path loss. First of all, the transmission distance affects the loss of RF signal power as a power loss is inversely proportional to a distance. Second factor is fading caused by multi-path propagation. One of fading induced by multi-path propagation is so-called frequency-selective fading. It happens when signals from different path are added, causing either constructive or destructive change on an amplitude and an phase of a signal at the receiver. The other major fading is slow fading, so-called shadowing. It is caused when a signal is refracted, reflected, and diffracted by obstacle [18].

Received signal strength is used in localization as a distance can be estimated from the power received in the particular frequency channel. Therefore, the received signal strength indicator (RSSI) is designed in order to measure the power received from RF signal. The received signal strength indicator (RSSI) is implemented inside a sensor node so that external hardware is not required for measuring the power received. However, value on RSSI oscillates due to multi-path fading or mobile environment and so on [19].

By relation between power value measured on received signal strength indicator (RSSI) and the distance between nodes, the distance from transmitter to receiver can be estimated. In free space, signal power is inversely proportional to square of distance. In realistic channels, there are two main interferences in RSS measurement, multi-path signals and shadowing.

Multi-path signal fading takes place when multiple signals arrive with different amplitudes and phases at the receiver and theses signals are added positively and also negatively as a function of the frequency and distance. This distortion can be compensated using a spread-spectrum method averaging the received power over a wide bandwidth. The received power measured using wide bandwidth equals to the sum of the powers measured from each multi-path signal [20]. Even assuming that multi-path fading problem is solved, shadowing, environment dependent error, can also be problem.

Shadowing is attenuation of a signal due to obstructions like pillar, furniture, which a signal must pass through or diffract on the path between transmitter and receiver. Due to signals from various paths, it is more realistic to calculate the ensemble mean received power of all these signals. And signal distribution under fading channels has been proved to conform to a log-normal distribution closely [16].

Thus the mean power at distance $d$ is typically expressed as log-normal path loss propagation model by using a path loss exponent, which is $\bar{P}(d)=$ $P_{0}-10 n_{p} \log \frac{d}{d_{0}}$, where $P_{0}$ and $n_{p}$ denote the received power and the path-loss exponent respectively. A value of path loss exponent varies depending on the environment where communication takes place. For example, path loss exponent is between 2.7 and 3.5 for urban area cellular radio whereas it is between 2 and 3 for 
impaired circumstance in building [17]. The received power $P_{i, j}$ in $\mathrm{dBm}$ at sensor $i$ transmitted by $j$, can be expressed with a noise, which is Gaussian distribution with zero mean as

$$
f\left(P_{i, j}=p \mid \theta\right)=\mathcal{N}\left(p ; \bar{P}\left(d_{i, j}\right), \sigma_{\mathrm{dB}}^{2}\right)
$$

where $p, \bar{P}\left(d_{i, j}\right), \sigma_{d B}^{2}$ represent Gaussian probability density(pdf), mean power, and variance in $d B$ respectively.

Therefore, the simulation conducted in Chapter 3 applies formula $\bar{P}(d)=$ $P_{0}-10 n_{p} \log \frac{d}{d_{0}}+n$, where $n$ denotes Gaussian noise with standard deviation $\sigma_{\mathrm{dB}}$. More detailed explanation about parameter value such as received power at reference distance $P_{0}$ and path-loss exponent $n_{p}$ and $\sigma_{\mathrm{dB}}$ and so on is left to Section 3.1.2.

\subsubsection{Time of arrival}

A time of arrival (TOA) is the measured time at which a signal first arrives at a receiver. The time delay is computed by subtracting the known transmit time from the measured TOA. Sensors chosen for TOA have synchronized clocks. Two-way time of arrival measurement is more desirable than one-way time of arrival due to the fact that time synchronization is not necessary [18]. As we can see in Figure 2.2 [21], offset time, which is denoted as OS, is canceled out for two-way TOA calculation.

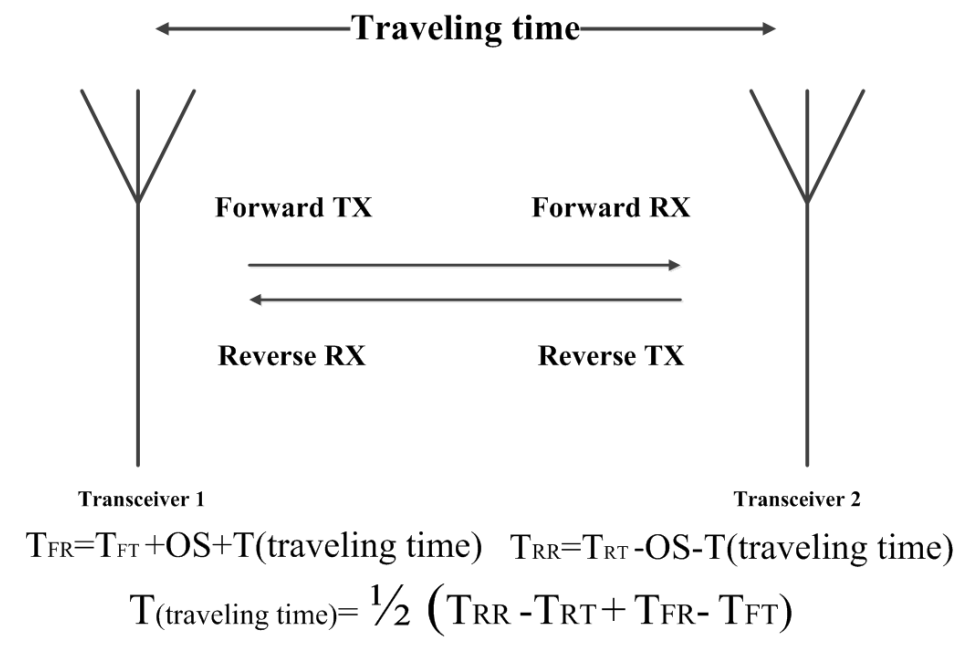

Figure 2.2: Time of arrival

The accuracy of the arrival time is limited by additive noise. In estimating accurate time of arrival (TOA), therefore, it is the key issue to find the time delay between the transmitted signal and the received signal distorted by noise as accurately as possible. However, estimation technique of time delay caused by additive noise is developed pretty well both in high and in low signal-to-noise case [22]. Developed technique is the generalized cross-correlation algorithm (the maximum 
likelihood estimator), which maximizes the signal to noise ratio [23]. And to get the general idea of reducing noise, it is helpful to look the formula of Cramér-Rao lower bound as it is the possible lowest variance. The Cramér-Rao lower bound in multipath-free channel is calculated as

$$
\begin{aligned}
& \frac{1}{8 \pi^{2} \cdot T \cdot W \cdot f_{r m s}^{2}} \cdot \frac{1+2 \cdot \mathrm{SNR}}{\mathrm{SNR}^{2}} \\
\simeq & \frac{1}{8 \pi^{2} \cdot T \cdot W \cdot f_{r m s}^{2}} \cdot \frac{2}{\mathrm{SNR}} \quad \text { for high SNR }
\end{aligned}
$$

where $T, W, f_{r m s}$ denotes the observation time, the bandwidth of the signal, RMS value of the center frequency of the signal respectively in a multi-path-free channel [24]. And $f_{r m s}$ is computed as $f_{r m s} \sqrt{1+\frac{W}{12 f_{c}^{2}}}$. As shown in the equation above, accuracy of TOA depends only on signal's bandwidth, the center frequency, observation time, and signal-to-noise ratio (SNR). Cramér-Rao lower bound (CRB) gives an idea which factors affect TOA and how those factors are related to the accuracy of TOA estimate [25].

The error caused by multi-path fading, another main interference, can be much more severe than that caused by additive noise. All multi-path components decreases the SNR of the desired line-of-sight signal. A line of sight refers to electro-magnetic wave traveling in straight line, not diffracted, reflected or shadowed by unexpected obstacles. In the multi-path channel, the receiver must find the first-arriving peak because it is not assured that the line-of-sight signal will be the strongest of the arriving signals. This measurement can be done by calculating time that the crosscorrelation crosses a threshold first. Template-matching is an alternative method to measure the time of arrival in multi-path channel [26].

Since dense sensor networks have the distinct advantage of being able to calculate TOA between nearby neighbors, the shorter inter-sensor distances are the less attenuated line-of-sight (LOS) signal is. And also, wider signal bandwidths provides narrower autocorrelation peak, which enhances the ability to accurately indicate the arrival time of a signal and makes it easy in distinguishing LOS signal from multipath signals [18].

In two-way time of arrival, since same measurement is used for calculating time, same clock offset will be applied for both forward (additive term) and reverse measurement (subtractive term) results as shown in figure above. Therefore, summation of forward and reverse offset is canceled out [27].

\subsection{Position estimation algorithm}

This section introduces several localization algorithms. First, least squares estimation, one of most known estimator, is introduced. Next, joint map estimation, possible algorithm for situation where an anchor is not given, is explained. And finally, the cooperative algorithms, the algorithms that utilize communication data between agent nodes in estimating location, is introduced. 


\subsubsection{Least squares estimation}

Least squares estimation (LSE) is the most common standard approach to find the "best fit" solution, where number of equations are more than a number of unknown variables [28]. For most cases, there is no solution $\mathbf{x}$ satisfying $\mathbf{A x}=\mathbf{b}$, where $\mathbf{A}$ and $\mathbf{b}$ are a design vector and an observed data vector respectively. Therefore, LSE is used to estimate value of $\mathbf{x}$ in a way that $\mathbf{A x}$ minimizes the euclidean distance between $\mathbf{b}$ and the column space of $\mathbf{A x}$. To express Ax mathematically, it can be

$$
\begin{aligned}
\mathbf{A} \mathbf{x} & =\left[\begin{array}{cccc}
a_{1,1} & a_{1,2} & \cdots & a_{1, n} \\
a_{2,1} & a_{2,2} & \cdots & a_{2, n} \\
\vdots & \vdots & \ddots & \vdots \\
a_{m, 1} & a_{m, 2} & \cdots & a_{m, n}
\end{array}\right]\left[\begin{array}{c}
x_{1} \\
x_{2} \\
\vdots \\
x_{n}
\end{array}\right]=\left[\begin{array}{c}
a_{1,1} \cdot x_{1}+a_{1,2} \cdot x_{2}+\cdots+a_{1, n} \cdot x_{n} \\
a_{2,1} \cdot x_{1}+a_{2,2} \cdot x_{2}+\cdots+a_{2, n} \cdot x_{n} \\
\vdots \\
a_{m, 1} \cdot x_{1}+a_{m, 2} \cdot x_{2}+\cdots+a_{m, n} \cdot x_{n}
\end{array}\right] \\
& =\left[\mathbf{a}_{1}\right] \cdot x_{1}+\left[\mathbf{a}_{2}\right] \cdot x_{2}+\cdots+\left[\mathbf{a}_{n}\right] \cdot x_{n}
\end{aligned}
$$

where $\mathbf{a}_{n}$ denotes $n$th column vector of $\mathbf{A}$. As proven above, $\mathbf{A x}$ is linear combinations of A's column space. In other words, $\mathbf{A x}$ is necessarily in the column space of A. According to The Best Approximation Theorem [28], if the column space of $\mathbf{A}$ is subspace of $\mathbb{R}^{m}$ and $\mathbf{b}$ is the vector in $\mathbb{R}^{m}$, then

$$
\|\mathbf{b}-\hat{\mathbf{b}}\|<\|\mathbf{b}-\mathbf{w}\|
$$

where $\hat{\mathbf{b}}$ is the perpendicular projection of $\mathbf{b}$ onto $\mathbf{w}$, any vector in the column space of $\mathbf{A}$. In other words, $\hat{\mathbf{b}}$ is closest point in A's column space to $\mathbf{b}$.

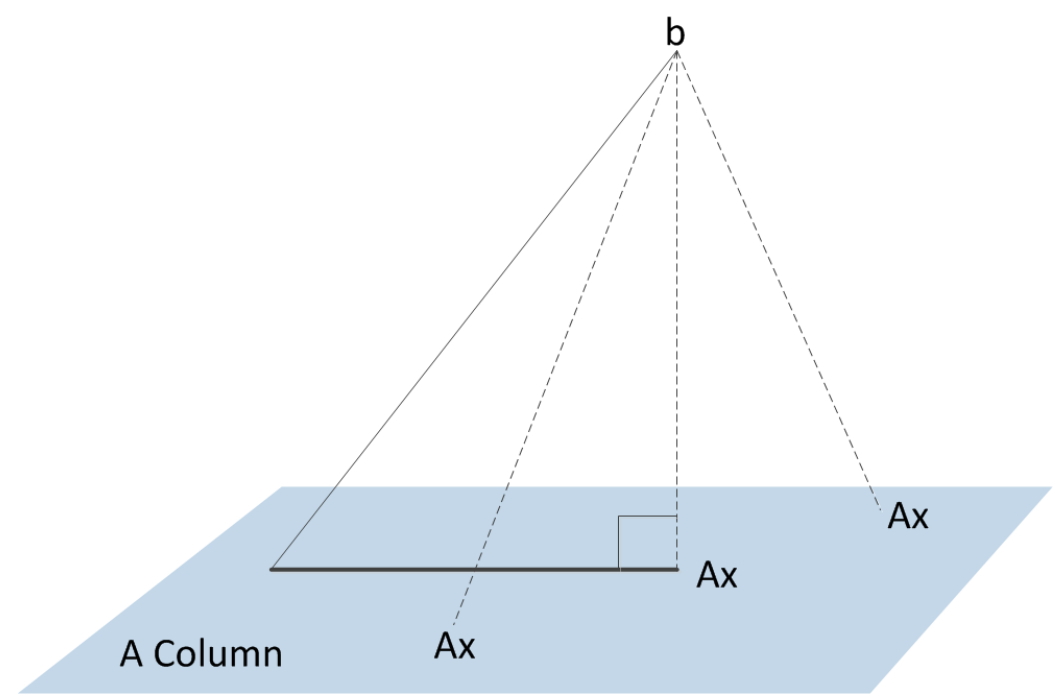

Figure 2.3: The closest point to the vector space

Figure 2.3 might help to understand why the perpendicular projection of $\mathbf{b}$ is the closest point of the column space of $\mathbf{A}$. Since $\mathbf{A x}$ lies in the column space of $\mathbf{A}$ 
regardless of $\mathbf{x}$, there always exists $\mathbf{x}$ satisfying $\mathbf{A} \mathbf{x}=\hat{\mathbf{b}}$. To find $\mathbf{x}$ which makes $\mathbf{A x}$ equals to the perpendicular projection of to column space of $\mathbf{A} \hat{\mathbf{b}}$, we need to use orthogonal property of two vectors which $\mathbf{b}$ is composed of.

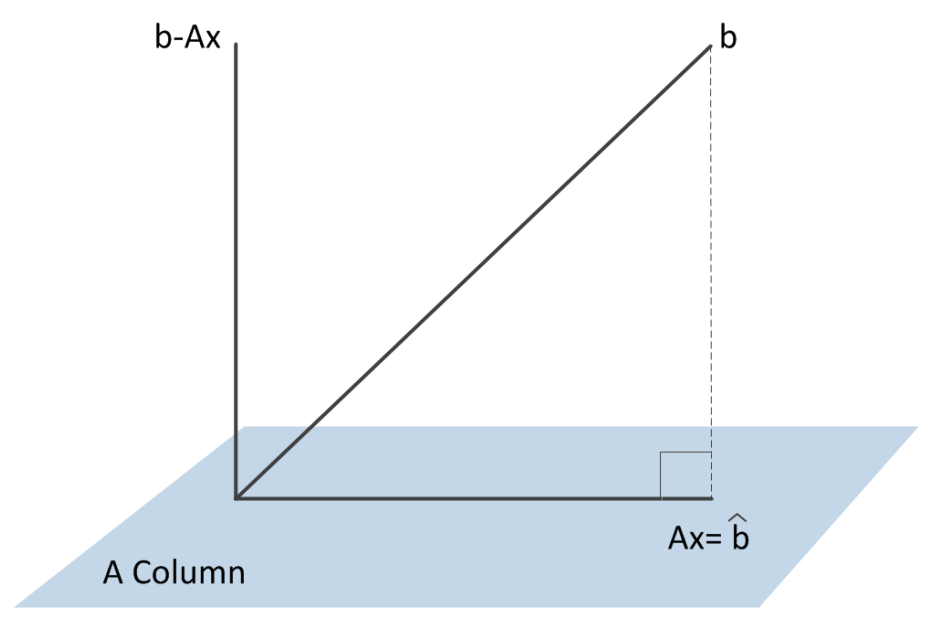

Figure 2.4: The orthogonal decomposition

As shown in Figure 2.4, $\mathbf{b}$ can be decomposed into the vector $\mathbf{b}-\mathbf{A x}$ and $\mathbf{A x}$, which lies in column space of $\mathbf{A}$. And these two vectors are orthogonal to each other only when $\mathbf{A x}=\mathbf{b}$. Therefore, the goal is to find $\mathbf{x}$ which satisfies $(\mathbf{b}-\mathbf{A x}) \cdot \mathbf{a}_{\mathrm{col}}=0$ where $\mathbf{a}_{\text {col }}$ denotes a column vector of $\mathbf{A}$. It can be expressed as below.

$$
\begin{gathered}
\mathbf{A}^{\top}(\mathbf{b}-\mathbf{A} \mathbf{x})=0 \\
\text { Then, } \mathbf{A}^{\top} \mathbf{b}-\mathbf{A}^{\top} \mathbf{A} \mathbf{x}=0 \rightarrow \mathbf{A}^{\top} \mathbf{A} \mathbf{x}=\mathbf{A}^{\top} \mathbf{b}
\end{gathered}
$$

It is shown that the solution $\mathbf{x}$ of $\mathbf{A x}=\mathbf{b}$ is same as the solution $\mathbf{x}$ of $\mathbf{A}^{\top} \mathbf{A} \mathbf{x}=\mathbf{A}^{\top} \mathbf{b}$. Finally, according the Equation (2.6) the least squares solution is

$$
\mathbf{x}=\left(\mathbf{A}^{\top} \mathbf{A}\right)^{-1} \mathbf{A}^{\top} \mathbf{b}
$$

A simple example of least squares application, linear equation which is $y=\beta_{0}+\beta_{1} x$, can be drawn in 2-dimension as below.

As shown in figure 2.5, the difference between $y$ value of data and $y$ value of the line, the error, is called as residual. And least squares estimation works in a way that make the line as close as possible to $n$ data points $\left(x_{1}, y_{1}\right), \cdots,\left(x_{n}, y_{n}\right)$. Therefore, the purpose of this estimation is to find the parameters $\beta_{0}, \beta_{1}$ of the line $y=\beta_{1} \cdot x+\beta_{0}$ which is minimizing the sum of residuals' squares. To rewrite linear equation above in matrix equation, it can be expressed as $\mathbf{x} \boldsymbol{\beta}=\mathbf{y}$, where

$$
\mathbf{x}=\left[\begin{array}{cc}
1 & x_{1} \\
1 & x_{2} \\
\vdots & \vdots \\
1 & x_{n}
\end{array}\right], \quad \boldsymbol{\beta}=\left[\begin{array}{c}
\beta_{0} \\
\beta_{1}
\end{array}\right], \quad \mathbf{y}=\left[\begin{array}{c}
y_{1} \\
y_{2} \\
\vdots \\
y_{n}
\end{array}\right]
$$




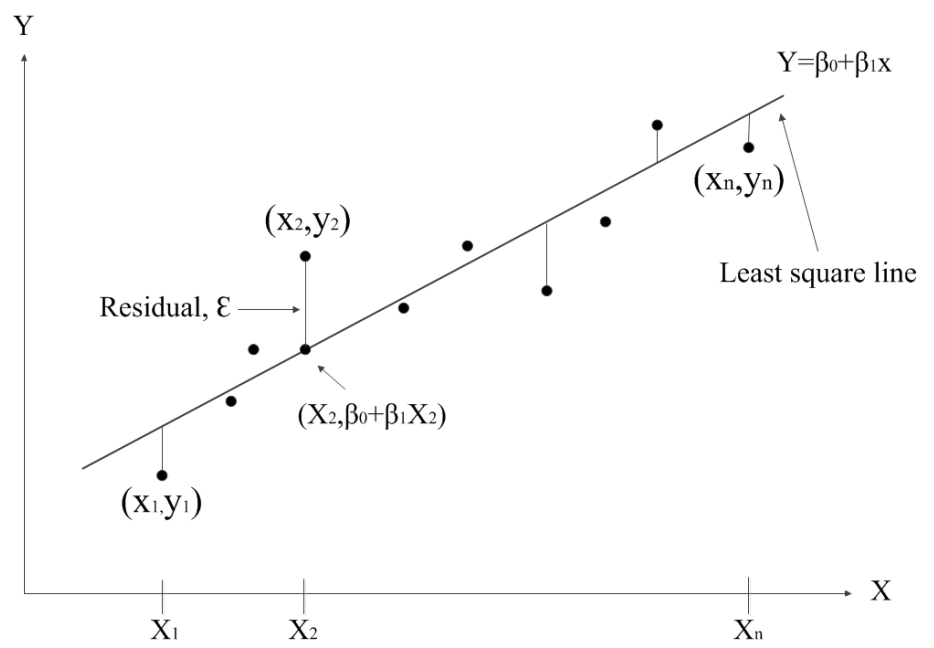

Figure 2.5: Fitting a line based on data

For the case of localization, since there are two unknown variables to be estimated; $x$ and $y$ coordinate of an unknown node, there should be at least more than three measured data of distances between the unknown node and anchor nodes. It is possible to calculate $x$ and $y$ coordinate of unknown node from square of distance $d^{2}$ between an unknown node and anchor nodes. In equation below, which derives position of unknown node from distance $d$, coordinates of anchor node $i$ is expressed as $x_{i}$ and $y_{i}$ respectively.

By using distance information, least squares estimation (LSE) can be used to find position of unknown node as below equations show in [29]. Let's start with three distance data, the simplest example, in order to get basic idea how general LSE equation is supposed to be

$$
\begin{aligned}
& \left(x_{1}-u_{x}\right)^{2}+\left(y_{1}-u_{y}\right)^{2}=d_{1}^{2} \\
& \left(x_{2}-u_{x}\right)^{2}+\left(y_{2}-u_{y}\right)^{2}=d_{2}^{2} \\
& \left(x_{3}-u_{x}\right)^{2}+\left(y_{3}-u_{y}\right)^{2}=d_{3}^{2}
\end{aligned}
$$

where $u_{x}$ and $u_{y}$ denote coordinates of unknown node. For calculation of distance based on measurements (RSS and TOA) is left to chapter 3. Under assumption that distance is obtained from measurements, formulas above can be expanded as below.

$$
\begin{aligned}
& x_{1}^{2}-2 x_{1} u_{x}+u_{x}^{2}+y_{1}^{2}-2 y_{1} u_{y}+u_{y}^{2}=d_{1}^{2} \\
& x_{2}^{2}-2 x_{2} u_{x}+u_{x}^{2}+y_{2}^{2}-2 y_{2} u_{y}+u_{y}^{2}=d_{2}^{2} \\
& x_{3}^{2}-2 x_{3} u_{x}+u_{x}^{2}+y_{3}^{2}-2 y_{3} u_{y}+u_{y}^{2}=d_{3}^{2}
\end{aligned}
$$

Equations above need to be subtracted to one another to remove $n_{x}^{2}$ and $n_{y}^{2}$. Then, equation will be reduced as below.

$$
\begin{aligned}
& \left(x_{1}^{2}-x_{2}^{2}\right)-2 u_{x}\left(x_{1}-x_{2}\right)+\left(y_{1}^{2}-y_{2}^{2}\right)-2 u_{y}\left(y_{1}-y_{2}\right)=d_{1}^{2}-d_{2}^{2} \\
& \left(x_{1}^{2}-x_{3}^{2}\right)-2 u_{x}\left(x_{1}-x_{3}\right)+\left(y_{1}^{2}-y_{3}^{2}\right)-2 u_{y}\left(y_{1}-y_{3}\right)=d_{1}^{2}-d_{3}^{2}
\end{aligned}
$$




$$
\left(x_{2}^{2}-x_{3}^{2}\right)-2 u_{x}\left(x_{2}-x_{3}\right)+\left(y_{2}^{2}-y_{3}^{2}\right)-2 u_{y}\left(y_{2}-y_{3}\right)=d_{2}^{2}-d_{3}^{2}
$$

Then, move all of elements which are multiplied with neither $x$ nor $y$ coordinate of unknown node $\left(n_{x}, n_{y}\right)$ to the left-hand side.

$$
\begin{aligned}
& \left(x_{1}^{2}-x_{2}^{2}\right)+\left(y_{1}^{2}-y_{2}^{2}\right)-d_{1}^{2}+d_{2}^{2}=2 u_{x}\left(x_{1}-x_{2}\right)+2 u_{y}\left(y_{1}-y_{2}\right) \\
& \left(x_{1}^{2}-x_{3}^{2}\right)+\left(y_{1}^{2}-y_{3}^{2}\right)-d_{1}^{2}+d_{3}^{2}=2 u_{x}\left(x_{1}-x_{3}\right)+2 u_{y}\left(y_{1}-y_{3}\right) \\
& \left(x_{2}^{2}-x_{3}^{2}\right)+\left(y_{2}^{2}-y_{3}^{2}\right)-d_{2}^{2}+d_{3}^{2}=2 u_{x}\left(x_{2}-x_{3}\right)+2 u_{y}\left(y_{2}-y_{3}\right)
\end{aligned}
$$

Finally, those three equation can be expressed in matrix form as

$$
\mathbf{b}=\mathbf{A x}
$$

where

$$
\mathbf{b}=\left[\begin{array}{l}
x_{1}^{2}-x_{2}^{2}+y_{1}^{2}-y_{2}^{2}-d_{1}^{2}+d_{2}^{2} \\
x_{1}^{2}-x_{3}^{2}+y_{1}^{2}-y_{3}^{2}-d_{1}^{2}+d_{3}^{2} \\
x_{2}^{2}-x_{3}^{2}+y_{2}^{2}-y_{3}^{2}-d_{2}^{2}+d_{3}^{2}
\end{array}\right] \quad \mathbf{A}=\left[\begin{array}{ll}
2\left(x_{1}-x_{2}\right) & 2\left(y_{1}-y_{2}\right) \\
2\left(x_{1}-x_{3}\right) & 2\left(y_{1}-y_{3}\right) \\
2\left(x_{2}-x_{3}\right) & 2\left(y_{2}-y_{3}\right)
\end{array}\right] \quad \mathbf{x}=\left[\begin{array}{l}
u_{x} \\
u_{y}
\end{array}\right]
$$

Therefore, general LSE matrix where $n$ distance data is given can be expressed as

$$
\mathbf{b}=\left[\begin{array}{c}
x_{1}^{2}-x_{2}^{2}+y_{1}^{2}-y_{2}^{2}-d_{1}^{2}+d_{2}^{2} \\
\vdots \\
x_{1}^{2}-x_{n}^{2}+y_{1}^{2}-y_{n}^{2}-d_{1}^{2}+d_{n}^{2} \\
\vdots \\
x_{n-1}^{2}-x_{n}^{2}+y_{n-1}^{2}-y_{n}^{2}-d_{n-1}^{2}+d_{n}^{2}
\end{array}\right] \mathbf{A}=\left[\begin{array}{cc}
2\left(x_{1}-x_{2}\right) & 2\left(y_{1}-y_{2}\right) \\
\vdots & \vdots \\
2\left(x_{1}-x_{n}\right) & 2\left(y_{1}-y_{n}\right) \\
\vdots & \vdots \\
2\left(x_{n-1}-x_{n}\right) & 2\left(y_{n-1}-y_{n}\right)
\end{array}\right] \mathbf{x}=\left[\begin{array}{l}
x_{u} \\
y_{u}
\end{array}\right]
$$

Therefore, matrix $\mathbf{x}$, which is $\mathrm{x}, \mathrm{y}$ coordinate of unknown node can be calculated due to

$$
\begin{aligned}
& \mathbf{b}=\mathbf{A} \mathbf{x} \Rightarrow \mathbf{A} \mathbf{x}=\mathbf{b} \\
& \mathbf{x}=\left(\mathbf{A}^{\top} \mathbf{A}\right)^{-1} \mathbf{A}^{\top} \mathbf{b}
\end{aligned}
$$

\subsubsection{Joint map estimation}

Joint map estimation algorithm realizes localization using only the distance information between nodes in group $A$ and nodes in group $B$ without information of the distance between nodes in same node group [30]. Furthermore, the location of all nodes are unknown as well. However, in this thesis an anchor node (a node whose location is already known) is included to estimate absolute sensory location 
since localization using the distance information provides only a general map of the all sensors' relative locations, .

There are various scenarios where this algorithm can be applied. A scenario can be that a position of a node group $A$ is traveled by a mobile node and the location of a node group $B$ is fixed. Another possible scenario is that sensor node group $A$ and sensor node group $B$ are identical and all distance between nodes are identified.

This algorithm decomposes the distance matrix between sensor node group A and $\mathbf{B}$ into $x$ and $y$ coordinate of both sensor groups. To express mathematically, the squared distance matrix $\mathbf{D}$ is decomposed into matrix $\mathbf{A}$ and $\mathbf{B}$ where $\mathbf{A}$ and $\mathbf{B}$ is

$$
\mathbf{A}=\left[\begin{array}{llll}
\mathbf{1} & \sqrt{2} \mathbf{a}_{x} & \sqrt{2} \mathbf{a}_{y} & \mathbf{a}_{d}
\end{array}\right] \in \mathbb{R}^{N_{a} \times 4} \mathbf{B}=\left[\begin{array}{llll}
\mathbf{b}_{d} & -\sqrt{2} \mathbf{b}_{x} & -\sqrt{2} \mathbf{b}_{y} & \mathbf{1}
\end{array}\right] \in \mathbb{R}^{N_{b} \times 4}
$$

where $\mathbf{a}_{d}$ and $\mathbf{b}_{d}$ denotes $\mathbf{a}_{x} \cdot \mathbf{a}_{x}+\mathbf{a}_{y} \cdot \mathbf{a}_{y}$ and $\mathbf{b}_{x} \cdot \mathbf{b}_{x}+\mathbf{b}_{y} \cdot \mathbf{b}_{y}$ respectively. In addition, $N_{a}$ and $N_{b}$ denote a number of sensor node in group $A$ and $B$ respectively. The squared distance $\mathbf{D}$ can be expressed as

$$
\mathbf{D}=\mathbf{A B}^{\top}
$$

However, due to presented noise, the squared distance matrix calculated in noisy environment is very likely to be different from squared true distance. Therefore, square of measured distance, $\hat{\mathbf{D}}$, can be expressed as $\mathbf{A B}^{\top}+\sigma \mathbf{W}$ where $\mathbf{W}$ is normal distributed random variable matrix with unit variance.

After following the calculation as written in [30], one can get $x$ and $y$ coordinate of both $A$ and $B$ node groups. As nodes of $A$ and $B$ group are set to be identical in the simulation throughout this thesis, $x$ and $y$ coordinate of both node groups are same. In situation where a transmission range of all nodes is reachable to one another, cooperative localization can be realized by placing all unknown nodes not only one unknown node in either sensor group. In Chapter 4, the comparison between traditional and cooperative localization results is given.

However, since the locations of sensor nodes are just relative location, reformulation work is needed to convert a relative location in either sensor group into an absolute location using the location of anchor nodes. The structure of the relative location of anchor nodes matrix $\mathbf{R}$ is

$$
\mathbf{R}=\left[\begin{array}{ll}
\mathbf{r}_{x} & \mathbf{r}_{y}
\end{array}\right] \in \mathbb{R}^{N_{n} \times 2}
$$

where $N_{n}$ denotes a number of anchor nodes. Using the relative location of anchor nodes and the absolute location of anchor nodes $\mathbf{A}_{a} \in \mathbb{R}^{N_{n} \times 2}$, reformulation matrix $\mathbf{F} \in \mathbb{R}^{2 \times 3}$ can be computed as

$$
\mathbf{F R}_{a}=\mathbf{A}_{a}
$$


where

$$
\mathbf{R}_{a}=\left[\begin{array}{lll}
\mathbf{r}_{x} & \mathbf{r}_{y} & \mathbf{1}
\end{array}\right] \in \mathbb{R}^{N_{n} \times 3}
$$

The structure of reformulation matrix is

$$
\mathbf{F}=\left[\begin{array}{lll}
f_{11} & f_{12} & f_{13} \\
f_{21} & f_{22} & f_{23}
\end{array}\right]
$$

Elements in first row of $\mathbf{F}\left(f_{11}, f_{12}\right.$, and $\left.f_{13}\right)$ are $x, y$, and constant coefficient respectively, which reformulate the relative location to $x$ coordinate of the absolute location of anchor nodes. Likewise, second row of $\mathbf{F}$ reformulates the relative location to $y$ coordinate of the absolute location. Since $\mathbf{F R}_{a}=\mathbf{A}_{a}, \mathbf{F}$ is computed as

$$
\mathbf{F}=\mathbf{A}_{a} \mathbf{R}_{a}^{\top}\left(\mathbf{R}_{a} \mathbf{R}_{a}^{\top}\right)^{-1}
$$

With reformulation matrix $\mathbf{F}$ computed by the equation above, the relative location of all nodes including unknown nodes in either sensor group, $\mathbf{U} \in \mathbb{R}^{N_{a} \times 2}$ can be reformulated. And the structure of the relative location of all nodes $\mathbf{U}$ is

$$
\mathbf{U}=\left[\begin{array}{ll}
\mathbf{u}_{x} & \mathbf{u}_{y}
\end{array}\right] \in \mathbb{R}^{N_{a} \times 2}
$$

where $N_{a}$ is a number of all nodes in either sensor group since sensors in group $A$ and $B$ are set to be identical. Finally, the absolute location of all nodes can be found by computing $\mathbf{U}_{r} \mathbf{F}^{\top}$. where

$$
\mathbf{U}_{r}=\left[\begin{array}{lll}
\mathbf{u}_{x} & \mathbf{u}_{y} & \mathbf{1}
\end{array}\right] \in \mathbb{R}^{N_{a} \times 3}
$$

\subsubsection{Conjugate descent}

This section illustrates the conjugate descent algorithm, the cooperative localization algorithm used through. This algorithm updates unknown nodes iteratively from initial point until the position of undated unknown nodes is barely changed. First, we will go through how the gradient descent works since its mechanism is similar to that of conjugate descent and easier to understand than conjugate descent. After that, conjugate descent concept is introduced as a supplementary method to overcome the drawback of the gradient descent. More detailed explanation will be followed in following subsections.

\section{Gradient descent}

The gradient descent algorithm is the localization method based on partial derivative of the distance between nodes. It is often called as the steepest descent as minimizing error function moves in steepest direction, the direction of negative gradient. The gradient descent is operated iteratively until the accuracy is not 
improved any more [31]. This method is variation of the ordinary lest squares estimation, which localizes more precisely especially in the environment where the parameters to be estimated are formed in non-liner function such as localization under log- normal shadowing [32].

The object to be detected can be localized cooperatively, which is the way of localization based on distance information not only from anchor nodes but also from agent nodes within a transmission range contrary to least square estimation introduced in section 2.2.1. In this algorithm, the squared distance matrix $\mathbf{D}$ is formed as below.

$$
D=\left[\begin{array}{cccccc}
d^{2}\left(a_{1}, a_{1}\right) & \cdots & d^{2}\left(a_{1}, a_{m}\right) & d^{2}\left(a_{1}, u_{1}\right) & \cdots & d^{2}\left(a_{1}, u_{n}\right) \\
\vdots & \ddots & \vdots & \vdots & \ddots & \vdots \\
d^{2}\left(a_{m}, a_{1}\right) & \cdots & d^{2}\left(a_{m}, a_{m}\right) & d^{2}\left(a_{m}, u_{1}\right) & \cdots & d^{2}\left(a_{m}, u_{n}\right) \\
d^{2}\left(u_{1}, a_{1}\right) & \cdots & d^{2}\left(u_{1}, a_{m}\right) & d^{2}\left(u_{1}, u_{1}\right) & \cdots & d^{2}\left(u_{1}, u_{n}\right) \\
\vdots & \ddots & \vdots & \vdots & \ddots & \vdots \\
d^{2}\left(u_{n}, a_{1}\right) & \cdots & d^{2}\left(u_{n}, a_{m}\right) & d^{2}\left(u_{1}, u_{1}\right) & \cdots & d^{2}\left(u_{n}, u_{n}\right)
\end{array}\right]
$$

where $a_{i}$ and $u_{i}$ is $i$ th anchor node and $i$ th unknown node, respectively; and $d(a, b)$ denotes the distance to the node $b$ from the node $a$. In addition, $m$ and $n$ denote a number of anchor nodes and a number of unknown nodes respectively. The reason of putting square on distance is for convenience in calculation of partial derivative, which will be explained later. The goal of this algorithm is to minimize square of difference between squared observed and estimated distance matrix, which can be expressed as

$$
\left\|\mathbf{D}_{O}-\mathbf{D}_{E}\right\|_{F}^{2}=c \simeq 0
$$

where $\mathbf{D}_{O}, \mathbf{D}_{E}$, and $c$ is squared observed distance and squared estimated distance with respect to an unknown node, cost function to be minimized respectively. Observed distance indicates the distance between all reachable nodes and an unknown node's location, which is calculated based on measurement data such as time of arrival and received signal strength. And estimated distance is the distance between all reachable nodes and an unknown node's location, which can randomly set. To minimize the equation above, its derivation should be zero.

Therefore, the partial derivative of $\left\|\mathbf{D}_{O}-\mathbf{D}_{E}\right\|_{F}^{2}$ with respect to $x$ and $y$ coordinate of an estimated unknown node, $u_{x}$ and $u_{y}$, is desired to be zero. This algorithm is operated iteratively until there is no change on $c$. Partial derivative of $c \nabla c$ is mathematically expressed as

$$
\begin{aligned}
\nabla c & =\frac{1}{2} \cdot \nabla c=\frac{1}{2} \cdot\left\|\mathbf{D}_{O}-\mathbf{D}_{E}\right\|_{F}^{2^{\prime}}=\frac{1}{2} \cdot \sum_{i=1}^{m+n} \sum_{j=1}^{n}\left(\mathbf{D}_{O}^{(i, j)}-\mathbf{D}_{E}^{(i, j)}\right)^{2^{\prime}} \\
& =\frac{1}{2} \cdot 2 \cdot \sum_{i=1}^{m+n} \sum_{j=1}^{n}\left(\mathbf{D}_{O}^{(i, j)}-\mathbf{D}_{E}^{(i, j)}\right) \cdot \sum_{i=1}^{m+n} \sum_{j=1}^{n}\left(\mathbf{D}_{O}^{(i, j)}-\mathbf{D}_{E}^{(i, j)}\right)^{\prime} \\
& =\sum_{i=1}^{m+n} \sum_{j=1}^{n}\left(\mathbf{D}_{O}^{(i, j)}-\mathbf{D}_{E}^{(i, j)}\right) \cdot-1 \cdot \sum_{i=1}^{m+n} \sum_{j=1}^{n} \mathbf{D}_{E}^{(i, j)^{\prime}} \approx 0
\end{aligned}
$$


$\frac{1}{2}$ is multiplied to $\nabla c$ to ease the computation. By taking partial derivative of $\left\|\mathbf{D}_{O}-\mathbf{D}_{E}\right\|_{F}^{2}$ with respect to coordinate of estimated unknown node iteratively, a position of unknown nodes is estimated. Before calculation of partial derivative of how overall sum of an element in $\mathbf{D}_{E}, d_{E}^{2}$, it is helpful to know how it is expressed. And it is

$$
\begin{aligned}
d_{E}^{2}= & \sum_{t=1}^{m+n} \sum_{j=1}^{n} \mathbf{D}_{E}^{(t, j)} \\
& =\sum_{k=1}^{m} \sum_{j=1}^{n} d_{E}^{2}\left(a_{k}, u_{i}\right)+\sum_{l=1}^{n} \sum_{j=1}^{n} d_{E}^{2}\left(u_{l}, u_{j}\right) \\
& =\sum_{k=1}^{m} \sum_{j=1}^{n}\left(a_{\mathrm{xk}}-u_{\mathrm{xj}}\right)^{2}+\left(a_{\mathrm{yk}}-u_{\mathrm{yj}}\right)^{2}+\sum_{l=1}^{n} \sum_{j=1}^{n}\left(u_{\mathrm{xl}}-u_{\mathrm{xj}}\right)^{2}+\left(u_{\mathrm{yl}}-u_{\mathrm{yj}}\right)^{2}
\end{aligned}
$$

where $a_{x}$ and $a_{y}$ denote coordinate of an anchor node. In addition, $u_{x}$ and $u_{y}$ denote coordinate of an unknown node. Furthermore, the distance $d$ is expressed as $\sqrt{\left(x_{1}-x_{2}\right)^{2}+\left(y_{1}-y_{2}\right)^{2}}$ where $x_{i}$ and $y_{i}$ denote $i$ th $x$ and $y$ coordinate respectively. Therefore, partial derivative $\frac{\partial d_{E}^{2 \prime}}{\partial u_{x}}, \frac{\partial d_{E}^{2 \prime}}{\partial u_{y}}$ can be calculated respectively as below

$$
\begin{aligned}
& \frac{\partial d_{E}^{2}}{\partial u_{x}}=-2 \cdot \sum_{k=1}^{m} \sum_{j=1}^{n}\left(a_{\mathrm{xk}}-u_{\mathrm{xj}}\right)+2 \cdot \sum_{l=1}^{n} \sum_{j=1}^{n}\left(u_{\mathrm{xl}}-u_{\mathrm{xj}}\right) \\
& \frac{\partial d_{E}^{2}}{\partial u_{y}}=-2 \cdot \sum_{k=1}^{m} \sum_{j=1}^{n}\left(a_{\mathrm{yk}}-u_{\mathrm{yj}}\right)+2 \cdot \sum_{l=1}^{n} \sum_{j=1}^{n}\left(u_{\mathrm{yl}}-u_{\mathrm{yj}}\right)
\end{aligned}
$$

Since the equation above is the sum of all unknown nodes' partial derivative, partial derivative of one particular unknown node is expressed as

$$
\begin{aligned}
& \frac{\partial d_{E}^{2}}{\partial u_{\mathrm{xi}}}=-2 \cdot \sum_{k=1}^{m}\left(a_{\mathrm{xk}}-u_{\mathrm{xi}}\right)+2 \cdot \sum_{j=1}^{n}\left(u_{\mathrm{xi}}-u_{\mathrm{xj}}\right) \\
& \frac{\partial d_{E}^{2}}{\partial u_{\mathrm{yi}}}=-2 \cdot \sum_{k=1}^{m}\left(a_{\mathrm{yk}}-u_{\mathrm{yi}}\right)+2 \cdot \sum_{j=1}^{n}\left(u_{\mathrm{yi}}-u_{\mathrm{yj}}\right)
\end{aligned}
$$

Finally partial derivative of $c$, with respect to coordinate of $i$ th unknown node $u_{x} i$ and $u_{y} i$ can be calculated. In this calculation, $\frac{1}{2}$ is also multiplied for computational convenience as done in Equation (2.29). Therefore partial derivative of $c$ is computed 


$$
\begin{aligned}
& \frac{\partial c}{\partial u_{\mathrm{xi}}} \\
= & \frac{1}{2} \cdot \sum_{t=1}^{m+n}\left(\mathbf{D}_{O}^{(t, i)}-\mathbf{D}_{E}^{(t, i)}\right) \cdot(-1) \cdot \frac{\partial d_{E}^{2}}{\partial u_{\mathrm{xi}}} \\
= & \frac{1}{2} \cdot \sum_{t=1}^{m+n}\left(\mathbf{D}_{O}^{(t, i)}-\mathbf{D}_{E}^{(t, i)}\right) \cdot(-1) \cdot\left(-2 \cdot \sum_{k=1}^{m}\left(a_{\mathrm{xk}}-u_{\mathrm{xi}}\right)+2 \cdot \sum_{j=1}^{n}\left(u_{\mathrm{xi}}-u_{\mathrm{xj}}\right)\right) \\
= & \sum_{t=1}^{m+n}\left(\mathbf{D}_{O}^{(t, i)}-\mathbf{D}_{E}^{(t, i)}\right) \cdot\left(\sum_{k=1}^{m}\left(\mathrm{a}_{\mathrm{xk}}-u_{\mathrm{xi}}\right)-\sum_{j=1}^{n}\left(u_{\mathrm{xi}}-u_{\mathrm{xj}}\right)\right)
\end{aligned}
$$

This term can be expanded to give the final equation

$$
\begin{aligned}
& \frac{\partial c}{\partial u_{x i}} \\
= & \sum_{t=1}^{m+n}\left(\mathbf{D}_{O}^{(t, i)}-\mathbf{D}_{E}^{(t, i)}\right) \cdot\left(\sum_{k=1}^{m}\left(a_{\mathrm{xk}}-u_{\mathrm{xi}}\right)-\sum_{j=1}^{n}\left(u_{\mathrm{xi}}-u_{\mathrm{xj}}\right)\right) \\
= & \sum_{k=1}^{m}\left(d_{O}^{2}\left(a_{k}, u_{i}\right)-d_{E}^{2}\left(a_{k}, u_{i}\right)\right)+\sum_{j=1}^{n}\left(d_{O}^{2}\left(u_{i}, u_{j}\right)-d_{E}^{2}\left(u_{i}, u_{j}\right)\right) . \\
& \left(\sum_{k=1}^{m}\left(a_{\mathrm{xk}}-u_{\mathrm{xi}}\right)-\sum_{j=1}^{n}\left(u_{\mathrm{xi}}-u_{\mathrm{xj}}\right)\right) \\
= & \sum_{k=1}^{m}\left(d_{O}^{2}\left(a_{k}, u_{i}\right)-d_{E}^{2}\left(a_{k}, u_{i}\right)\right) \cdot \sum_{k=1}^{m}\left(a_{\mathrm{xk}}-u_{\mathrm{xi}}\right) \\
& -\sum_{j=1}^{n}\left(\left(d_{O}^{2}\left(u_{i}, u_{j}\right)-d_{E}^{2}\left(u_{i}, u_{j}\right)\right) \cdot \sum_{j=1}^{n}\left(u_{\mathrm{xi}}-u_{\mathrm{xj}}\right)\right. \\
= & \sum_{k=1}^{m}\left(d_{O}^{2}\left(a_{k}, u_{i}\right)-d_{E}^{2}\left(a_{k}, u_{i}\right)\right) \cdot\left(a_{\mathrm{xk}}-u_{\mathrm{xi}}\right) \\
& -\sum_{j=1}^{n}\left(d_{O}^{2}\left(u_{i}, u_{j}\right)-d_{E}^{2}\left(u_{i}, u_{j}\right)\right) \cdot\left(u_{\mathrm{xi}}-u_{\mathrm{xj}}\right)
\end{aligned}
$$

Since the result of formula above is to be minimized to zero, multiplicative factor 2 is taken away from formula as seen in last term in Equation (2.34). And likewise, 
$\frac{\partial c}{\partial u_{y i}}$ can be computed as

$$
\begin{aligned}
& \frac{\partial c}{\partial u_{\mathrm{yi}}}=\frac{1}{2} \cdot \sum_{t=1}^{m+n}\left(\mathbf{D}_{O}^{(t, i)}-\mathbf{D}_{E}^{(k, i)}\right) \cdot(-1) \cdot \frac{\partial d_{E}^{2}}{\partial u_{\mathrm{yi}}} \\
= & \sum_{k=1}^{m}\left(d_{O}^{2}\left(a_{k}, u_{i}\right)-d_{E}^{2}\left(a_{k}, u_{i}\right)\right) \cdot\left(a_{\mathrm{yk}}-u_{\mathrm{yi}}\right) \\
& -\sum_{j=1}^{n}\left(d_{O}^{2}\left(u_{i}, u_{j}\right)-d_{E}^{2}\left(u_{i}, u_{j}\right)\right) \cdot\left(u_{\mathrm{yi}}-u_{\mathrm{yj}}\right)
\end{aligned}
$$

Finally, the estimation of an unknown node $\left(u_{x}, u_{y}\right)$ being updated can be mathematically expressed as

$$
\left[\begin{array}{l}
u_{x} \\
u_{y}
\end{array}\right]_{\text {Update }}=\left[\begin{array}{l}
u_{x} \\
u_{y}
\end{array}\right]_{\text {Current }}-\mathrm{K}\left[\begin{array}{l}
\frac{\partial c}{\partial u_{x}} \\
\frac{\partial c}{\partial u_{y}}
\end{array}\right]
$$

where $(x, y)_{\text {Update }}$ and $(x, y)_{\text {Current }}$ is $x$ and $y$ coordinate of the unknown node's location to be updated and the current location respectively. In addition, $\mathrm{K}$ denotes step size which plays an important role in updating current location in a way that approaches to the true location correctly.

Despite its high accuracy, the gradient descent algorithm has critical drawback. The adequate step size has to be decided to get a high accuracy, which is hard to find optimal step size. Furthermore, initial point should be chosen well. In some case, due to incorrect initial point, convergence to the closest point to true location is not possible even after infinite iteration [33]. Therefore, there needs to be other supplementary algorithm to complement the drawbacks of the gradient descent method mentioned above. In this paper, the conjugate gradient method is suggested as a supplementary method. In following subsection, the conjugate gradient method will be introduced.

\section{Extension from the gradient descent}

The gradient descent algorithm minimizes the difference between true point and estimated point slowly. And extension of this algorithm, a conjugate gradient converges much faster. It is because for gradient descent new direction vector is just orthogonal to that of previous point in reaching a true point. As a result, gradient descent based localization finally converges to closest point to true location in a zig zag manner.

On the other hand, for conjugate gradient the direction vector of initial point directly searches closest point to true location in first step of iteration by stretching quadratic contour space to circular contour space as it is seen in Figure 2.6. And for rest iteration, the estimated position approaches to minimum point with equal length along one right direction throughout $n$ step. 


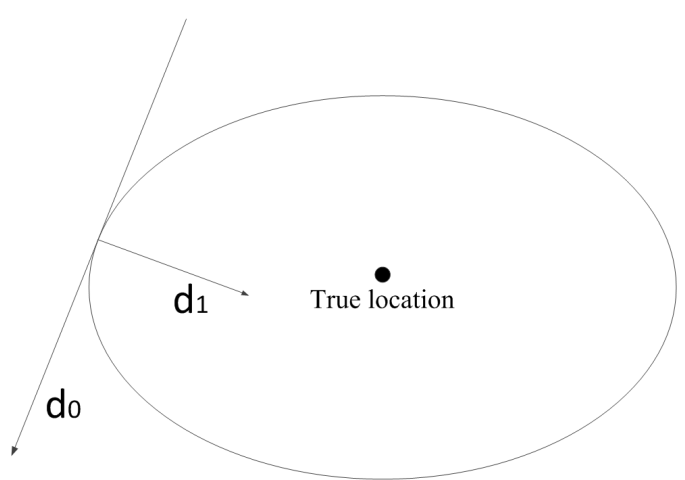

(a) Gradient descent

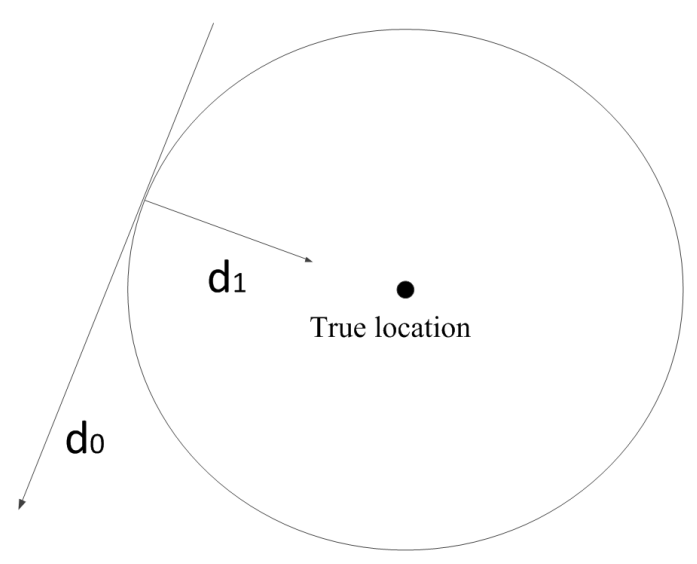

(b) Conjugate gradient

Figure 2.6: Comparison of direction vectors

In Figure 2.6, the orthogonal vector $d_{1}$ from previous direction vector $d_{0}$ is not able to search for the true location directly due to ellipse contour of object to be found in gradient descent. On the other hand, orthogonal vector $d_{1}$ from previous direction vector $d_{0}$ directly finds where true location is in conjugate gradient due to circular contour of true location. As a result, conjugate gradient makes it much faster in reaching the minimum point where the difference between estimated point and true location is smallest. However, it is still possible for conjugate gradient method not to make convergence on minimum point. The less quadratic function $J$ is, the higher chance the failure of convergence is [33].

As conjugate gradient is also a type of gradient methods its equation is similar to that of the gradient descent. The update of conjugate gradient for every step is basically same as the gradient descent method, which is

$$
\left[\begin{array}{l}
u_{x} \\
u_{y}
\end{array}\right]_{n+1}=\left[\begin{array}{l}
u_{x} \\
u_{y}
\end{array}\right]_{n}-K_{n} \cdot \mathbf{d}_{n}
$$

where, $K_{n}$ and $d_{n}$ indicates $n$th step coefficient, which determines how far current point should move along direction vector and $n$th step direction vector, which leads a algorithm in a way that approaches to minimum point directly. The conjugate gradient algorithm is designed in a way that direction vector at each iteration step is conjugate to one another.

To explain conjugacy concept, when there are $p$ and $q$ satisfying $\mathbf{p}^{\mathrm{T}} \mathbf{A q}=0 \mathbf{p}$ and $\mathbf{q}$ are said to be conjugate with respect to $\mathbf{A}$, which is any symmetric positive definite matrix. In this algorithm, $\mathbf{p}$ and $\mathbf{q}$ are two linearly independent direction vectors. And $\mathbf{A}$ is Hessian matrix, second order partial derivative matrix, of cost function explained in gradient descent part with respect to $x$ and $y$. To express mathematically, conjugate gradient descent satisfies $\mathbf{d}_{i}^{\mathrm{T}} \mathbf{H} \mathbf{d}_{j}=0$ at every step where $\mathbf{d}_{i}$ and $\mathbf{d}_{j}$ are previous and current direction vector respectively.

Among several conjugate gradient methods, scaled conjugate gradient is used for this paper due to its natural characteristic avoiding the complex computation of 
the Hessian by making simple but accurate approximation on second order of weigh vector of error function [34], [35]. Therefore, the scaled conjugate gradient has much faster than other conjugate gradient methods in computation.

\subsection{Cramér-Rao lower bound}

This section provides brief explanation about Cramér-Rao lower bound (CRLB). Next, the result of CRLB in cooperative localization based on TOA and RSS, the localizations to be implemented in the simulation part of thesis, is given.

Cramér-Rao lower bound (CRLB) is used as a mean for finding a lower bound on the covariance of any unbiased estimator relevant to measurement such as RSS or TOA. Since CRLB provides the best accuracy an unbiased estimator can reach, it has been useful for researchers and system designers who are concentrating on implementing localization algorithms, that is, CRLB is used as benchmark for checking of a particular algorithm's accuracy [18]. To grasp CRLB, it is very fruitful to know the theorem below [36].

Theorem Let $f(\mathbf{x} \mid \theta)$ be a family of pdfs parameterized by $\theta \in M$, the random sample number of $\mathbf{x}$. And also let's assume that $l$ denotes the log-likelihood function of $f(x)$ which is $\ln (f)$, and Fisher information matrix $J, J_{i j}$ equals to $E\left(\frac{\partial l}{\partial \theta_{i}} \frac{\partial l}{\partial \theta_{j}}\right)$. Given arbitrary coordinates $\theta=\left(\theta^{1}, \theta^{2}, \cdots, \theta^{n}\right) \in R^{n}$ on $M$, then for any unbiased estimator $\hat{\theta}$ of $\theta$

$$
C \geq J^{-1}
$$

where $C=E\left[(\theta-\hat{\theta})(\theta-\hat{\theta})^{\top}\right]$ is the error covariance matrix between an error $(\hat{\theta}-\theta)$ and $J$ is the Fisher information matrix with respect to these coordinates.

We will now observe the result of CRLB. Before going through results, there are some assumptions made to simplify the results of CRLB. The assumptions are [18]

First The localization conducted is two dimensional.

Second Received power strength and path loss exponent for RSS and time delay error for TOA are all known.

Under assumptions above, CRLB result of RSS and TOA is calculated. According to [18], CRLB matrix of RSS and TOA consists of three sub-matrices form $n \times n$, where $n$ indicates the number of unknown nodes. Three sub-matrices are

$$
\left[\mathbf{F}_{\mathrm{xx}}\right]_{k, l}= \begin{cases}\gamma \sum_{i \in H(k)}\left(x_{k}-x_{i}\right)^{2} / d_{k, i}^{s}, & k=l \\ -\gamma I_{H(k)}(l)\left(x_{k}-x_{l}\right)^{2} / d_{k, i}^{s}, & k \neq l\end{cases}
$$




$$
\begin{aligned}
& {\left[\mathbf{F}_{\mathrm{xy}}\right]_{k, l}= \begin{cases}\gamma \sum_{i \in H(k)}\left(x_{k}-x_{i}\right)\left(y_{k}-y_{i}\right) / d_{k, i}^{s}, & k=l \\
-\gamma I_{H(k)}(l)\left(x_{k}-x_{l}\right)\left(y_{k}-y_{l}\right) / d_{k, i}^{s}, & k \neq l\end{cases} } \\
& {\left[\mathbf{F}_{\mathrm{yy}}\right]_{k, l}= \begin{cases}\gamma \sum_{i \in H(k)}\left(y_{k}-y_{i}\right)^{2} / d_{k, i}^{s}, & k=l \\
-\gamma I_{H(k)}(l)\left(y_{k}-y_{l}\right)^{2} / d_{k, i}^{s}, & k \neq l\end{cases} }
\end{aligned}
$$

where $\gamma, \mathrm{s}, H(k)$, and $d_{a, b}$ denotes channel constant, exponent parameter, a set of sensors which are located inside transmission range of sensor $k$, and a distance between sensor $a$ and sensor $b$ respectively. In addition, $I_{H(k)}(l)$ is the indicator function, which allows the measurement information only if sensor $k$ makes a measurement with sensor $l$. For instance, if sensor $l$ satisfies $l \in H(k), I_{H(k)}(l)$ is 1 otherwise 0 .

A channel constant $\gamma$ and exponent parameter $s$ are varied on measurement type. In RSS case, a channel constant $\gamma$ is $\left(\frac{10 n_{p}}{\sigma_{d B} \log 10}\right)^{2}$, where $n_{p}$ is path-loss exponent and $\sigma_{d B}$ is standard deviation of received signal's power in $d B$ respectively. And the value of exponent parameter $s$ is 4 . On the other hand, in TOA measurement, a channel constant $\gamma$ is $1 /\left(v_{p} \sigma_{T}\right)^{2}$, where $v_{p}$ and $\sigma_{T}$ is wave propagation velocity and standard deviation of TOA respectively. And the value of exponent parameter $s$ is 2 .

For RSS and TOA case, three sub-matrices form entire Fisher information matrix $F$, inverse of CRLB, as

$$
\mathbf{F}=\left[\begin{array}{ll}
\mathbf{F}_{\mathrm{xx}} & \mathbf{F}_{\mathrm{xy}} \\
\mathbf{F}_{\mathrm{xy}}^{\top} & \mathbf{F}_{\mathrm{yy}}
\end{array}\right]
$$

As mentioned above, each of sub-matrix is $n \times n$. Therefore, Fisher information matrix $F$ must be $2 n \times 2 n$. Since CRLB is inverse of Fisher information matrix $F$, CRLB can be mathematically written as $F^{-1}$. $2 n$ diagonal elements of CRLB matrix are lower variance bound of $n$ unknown sensor; first $n$ for $x$ coordinate and last $n$ for $y$ coordinate. For example, the trace (main diagonal) of CRLB where has three unknown nodes can be displayed as

$$
\operatorname{tr}\left(\mathbf{F}^{-1}\right)=\left[\begin{array}{llllll}
x_{\mathrm{LB} 1} & x_{\mathrm{LB} 2} & x_{\mathrm{LB} 3} & y_{\mathrm{LB} 1} & y_{\mathrm{LB} 2} & y_{\mathrm{LB} 3}
\end{array}\right]
$$

where $x_{L B i}$ indicates lower bound of variance for $x$ coordinate of $i$ th unknown sensor. Therefore, variance of 1 st unknown sensor can be written as

$$
\sigma_{1}^{2} \geq x_{\mathrm{LB} 1}+y_{\mathrm{LB} 1}
$$

In consequence, the variance of $i$ th unknown sensor node out of $n$ unknown sensor nodes can be expressed as

$$
\sigma_{i}^{2} \geq \operatorname{tr}\left(\mathbf{F}^{-1}\right)_{i}+\operatorname{tr}\left(\mathbf{F}^{-1}\right)_{n+i}
$$




\section{Chapter 3}

\section{Setup and analysis for the simulation of various grid patterns}

This chapter conducts simulations of received signal strength (RSS) and time of arrival (TOA) based localizations. Both RSS and TOA based simulations are proceeded in the following order. First, two traditional (non-cooperative) localization algorithms, the location estimate method only using a distance information between an unknown node and an anchor node, are implemented in which anchor nodes are deployed in several particular grid patterns to be introduced later. After that, a cooperative localization, a localization method utilizing not only the information between an unknown node and an anchor node but also the data between unknown nodes, is conducted to prove its superior performance to traditional localization.

As a non-cooperative localization algorithm, least squares estimation and joint map estimation is used. And cooperative localization algorithm implemented in this thesis is scaled conjugate gradient, which is iterative least squares estimation. All algorithms are introduced in Chapter 2.

For each grid pattern, an accuracy of non-cooperative localization is calculated and evaluated as the density of sensor (number of sensor per $\mathrm{m}^{2}$ ) with the fixed transmission range first. After that, transmission range of a sensor node varies with the fixed sensor density.

\subsection{Set up and explanation of simulation}

This section illustrates the scenario of both received signal strength (RSS) and time of arrival (TOA)-related simulation to be conducted and shows how accuracy is computed in this simulation. Then, the detail explanation about formula and parameters used for RSS and TOA of the simulations are followed. 


\subsubsection{Scenario and process}

The scenario for both received signal strength (RSS) and time of arrival (TOA) based simulation is that unknown nodes are localized in the squared area where anchor nodes are deployed in three different grid pattern (square, random and triangle) as shown in figure 3.1 .

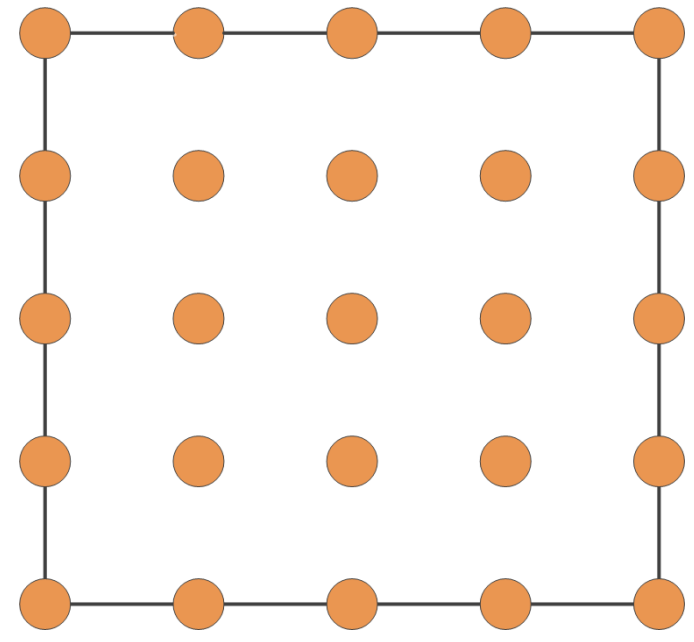

(a) Square grid

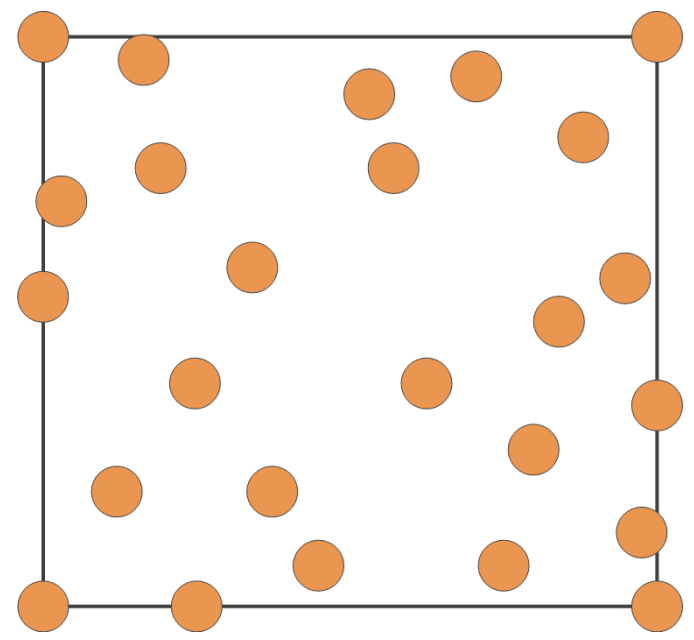

(b) Random grid

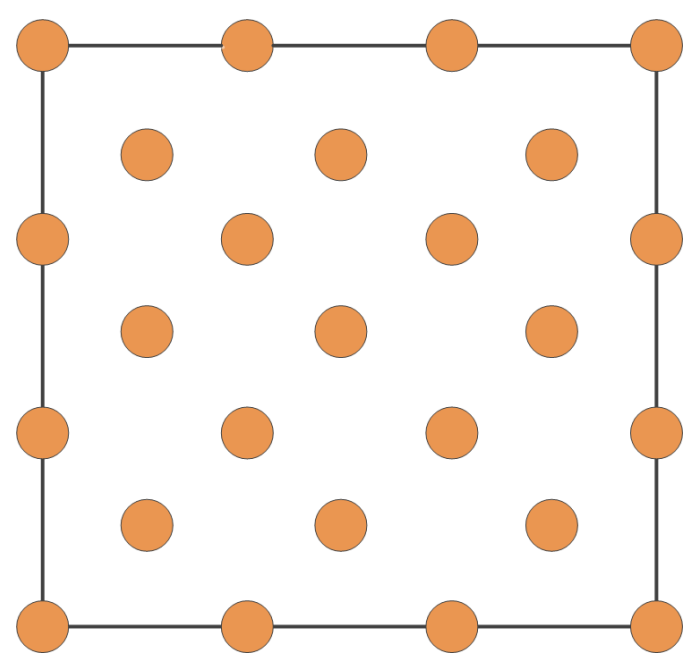

(c) Triangle grid

Figure 3.1: Anchor nodes deployed in three different grid patterns

The aim of deploying anchor nodes in three different grid patterns is to observe which anchor nodes' array maximizes the accuracy of the localization. It is very likely to get biased accuracy for one fixed random anchor node array. Therefore, the simulation is set in a way that random grid patterned anchor nodes are set to be re-arrayed while transmitting a signal many times. The more detail simulation setup about random grid pattern is in section 3.2.

In three different anchor node grid, unknown nodes are assumed to be randomly distributed in the smaller squared space in the center of where anchor nodes 
are located in as in figure 3.2. The reason of locating unknown node in smaller area is to avoid poor accuracy on the estimation of an unknown node located in the edge of the area.

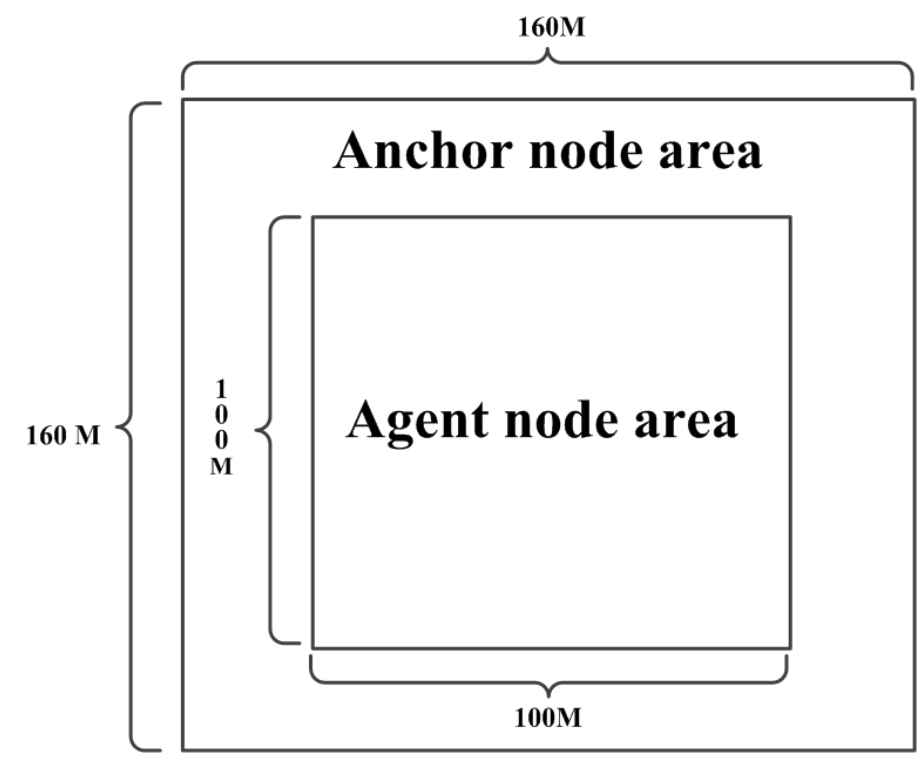

Figure 3.2: The area where anchor nodes and agent nodes are deployed

In both RSS and TOA simulations, every sensor sends a signal multiple times to other sensors located inside their transmission range. After transmitting a signal multiple times, the position of the unknown nodes is re-arrayed and a signal is transmitted multiple times again. This change of unknown nodes' formation is repeated several times. The reason for re-arraying unknown nodes' positions in computing accuracy is to avoid biased accuracy on a particular position of the unknown nodes. In other words, a signal is transmitted several times on each different formation of unknown nodes.

While the location of unknown nodes are changed, anchor nodes' array in random grid is also changed in a while to reduce the effect of bias accuracy on the certain random grid's position. The root mean squared error (RMSE) for every single signal transmission and the median value of RMSE collected is calculated. This process of RMSE computation is made in both scenario where a density of anchor nodes is varied with fixed transmission range and where a fixed transmission range is varied with fixed density of anchor node. Its detailed explanations is left to following subsections in this chapter.

Throughout this paper, a root mean square error (RMSE) based on two measurements (RSS and TOA) is shown as a mean to evaluate the accuracy of estimation. The RMSE is the square root of mean squared error, which is the difference between the estimated location and the true location. The RMSE is preferably used as the measure of the magnitude of errors in many fields. For instance, in electrical engineering field, root mean squared value is used to show the average value of AC voltage, which varies continuously [39]. If there are $n$ numbers of an estimated value 
$\theta$ towards to a true value $\mu$, root mean squared error (RMSE) is mathematically expressed as

$$
\sqrt{\frac{1}{n} \cdot\left(\left(\theta_{1}-\mu\right)^{2}+\left(\theta_{2}-\mu\right)^{2}+\cdots\left(\theta_{n}-\mu\right)^{2}\right)}=\theta_{r m s}
$$

Therefore, $n$ unknown nodes should be calculated as

$$
\sqrt{\frac{1}{n} \sum_{i=1}^{n}\left(\frac{\left(\sigma_{x i}^{2}\right)+\left(\sigma_{y i}^{2}\right)}{2}\right)}=U_{r m s}
$$

where $\sigma_{x i}^{2}, \sigma_{y i}^{2}, U_{r m s}$ denotes variance of $x$ and $y$ coordinate of $i$ th sensor node and average RMSE of $n$ number of unknown nodes respectively. And in most of simulation result in this thesis, $U_{r m s}$ for every single signal transmission has been collected and displayed in order to show the accuracy and the stability of localization results. Later section shows how the accuracy and the stability can be observed from the simulation results. Likewise, average root mean squared value of Cramér-Rao lower bound (CRLB) can be computed as [38]

$$
\sqrt{\frac{1}{2 \cdot n} \sum_{i=1}^{n}\left(\mathbf{F}_{x i}^{-1}+\mathbf{F}_{y i}^{-1}\right)}=U_{\mathrm{CRB}}
$$

where $\mathbf{F}_{x i}^{-1}$ and $\mathbf{F}_{y i}^{-1}$ denotes lower variance bound of $x$ and $y$ coordinate, which is the $i$ th and $n+i$ diagonal element of $\mathbf{F}^{-1}$ respectively as illustrated in 2.43 .

When calculating RMSE of localization of overall signals transmitted using $U_{r m s}$, it can be mathematically expressed as

$$
\sqrt{\frac{1}{N_{S} \cdot N_{C}} \sum_{j=1}^{N_{C}} \sum_{i=1}^{N_{S}} U_{r m s i}^{2}{ }^{(j)}}
$$

where $N_{S}$ is a number of signals transmitted and $N_{C}$ different array of unknown nodes.

When calculating CRLB of overall signals transmitted, standard deviation $\sigma$ of signals transmitted and the position of sensor nodes are key factor affecting a value of CRLB as shown in CRLB part in Chap.2. Therefore, average root mean squared CRLB of overall signals transmitted can be calculated as

$$
\sqrt{\frac{1}{2 \cdot N_{C}} \sum_{i=1}^{N_{C}} U_{\mathrm{CRB} i}^{2}}
$$

where $N_{C}$ is a number of different formulation of unknown nodes. Both average root mean squared error (RMSE) and average root mean squared CRLB of overall signals transmitted are given in the end of simulation part in order to give an overview of general accuracy of all algorithms. 
$\left[x_{u}, y_{u}\right]$ for all $\mathrm{u} \in \mathrm{U}$. In addition, a noise is now considered, which is more realistic. Therefore, the received signal power in noisy environment from each anchor node can be formulated as below

$$
P_{r}(i, k)=-20-20 \cdot \log _{10} \sqrt{\left(x_{\mathrm{ui}}-x_{\mathrm{ak}}\right)^{2}+\left(y_{\mathrm{ui}}-y_{\mathrm{ak}}\right)^{2}}+n
$$

where $P_{r}(i, k), n$ is the received signal power of $i$ th agent node from $k$ th anchor node and Gaussian noise respectively. In addition, $n$ satisfies $n \in N\left(0, \sigma^{2}\right)$ indicating normal distribution with zero mean and variance $\sigma^{2}$. From formula above, the distance can be easily computed from received power. For simplicity, other parts than $\sqrt{\left(x_{\mathrm{ui}}-x_{\mathrm{ak}}\right)^{2}+\left(y_{\mathrm{ui}}-y_{\mathrm{ak}}\right)^{2}}$ of the equation are all constant value. In addition, $\sqrt{\left(x_{\mathrm{ui}}-x_{\mathrm{ak}}\right)^{2}+\left(y_{\mathrm{ui}}-y_{\mathrm{ak}}\right)^{2}}$ is replaced by the true distance between node $i$ and node $k, d_{i, k}$. Finally, signal $P_{r}(i, k)$ is

$$
P_{r}(i, k)=C-10 \cdot n_{p} \cdot \log _{10} d_{i, k}+n
$$

where $C$ denotes a constant. Therefore, received power between any two locations $P_{r}$ can be expressed as

$$
\begin{aligned}
P_{r} & =C-10 \cdot n_{p} \cdot \log _{10} d+n \\
& =C-10 \cdot n_{p} \cdot \log _{10} \tilde{d}
\end{aligned}
$$

where, $d$ and $\tilde{d}$ denote the true distance and observed distance in noisy environment respectively. Since base 10 logarithm of distance measured from received power in noisy environment is mathematically expressed as

$$
\log _{10} \tilde{d}=\frac{C-P_{r}}{10 \cdot n_{p}}
$$

The observed distance $\tilde{d}$ under log-normal shadowing environment can be calculated as

$$
\begin{aligned}
\tilde{d} & =10^{\frac{C-P_{r}}{10 \cdot n_{p}}} \\
& =10^{\frac{C-\left(C-10 \cdot n_{p} \cdot \log _{10} d+n\right)}{10 \cdot n_{p}}} \\
& =10^{\frac{10 \cdot n_{p} \cdot \log _{10} d-n}{10 \cdot n_{p}}} \\
& =10^{\frac{10 \cdot n_{p} \cdot \log _{10} d}{10 \cdot n_{p}}} \cdot 10^{-\frac{n}{10 \cdot n_{p}}} \\
& =d \cdot 10^{-\frac{1}{10 \cdot n_{p}}} \propto d
\end{aligned}
$$

As shown in the equation above, the received power of the distance is affected by log-normal noise. Therefore, the noise affected to distance is multiplicative noise , $10^{-\frac{n}{10 \cdot n_{p}}}$, as shown in the equation above. The difference between observed distance and true distance (error) is $d \cdot\left(10^{-\frac{n}{10 \cdot n_{p}}}-1\right)$. Since $\frac{1}{10 \cdot n_{1}}$ is constant value and $n$ denotes random Gaussian noise, the error is proportional to true distance $d$. In other words, the longer transmission distance is, the bigger the gap between the true distance and the observed distance is. 


\subsubsection{Set-up for time of arrival}

This section explains about time of arrival (TOA) based simulation set-up such as parameter values and formula used in simulation. In addition, how the simulated data are modeled is shown. For time of arrival simulation, parameter values including variance are in table 3.2, which is example given in [18].

\begin{tabular}{|c|l|c|}
\hline \multicolumn{3}{|c|}{ Parameter of time of arrival } \\
\hline $\mathrm{v}_{\mathrm{p}}$ & propagation velocity & $3 \cdot 10^{8}(\mathrm{~m} / \mathrm{s})$ \\
\hline$\mu_{t}$ & mean of time delay & $0.3 \mathrm{~ns}$ \\
\hline$\sigma_{t}$ & Standard deviation of time delay & $6.1 \mathrm{~ns}$ \\
\hline
\end{tabular}

Table 3.2: Parameter values for TOA based simulation

According to [18] a noise in the TOA based localization can be roughly modeled as Gaussian distribution, whereas the channel based on RSS measurement experiences log-normal fading. Furthermore, the value of time delay variance is given value from [37]. Therefore, time of arrival data is generated based on that variance. In this simulation, two-way time of arrival (TOA) method is chosen in calculating TOA since it does not require time synchronization between a transmitter and a receiver. A time of arrival can be computed as

$$
\tau=\frac{1}{2} \cdot\left(T_{\mathrm{rr}}-T_{\mathrm{rt}}+T_{\mathrm{fr}}-T_{\mathrm{ft}}\right)
$$

where $T_{\mathrm{rr}}, T_{\mathrm{rt}}, T_{\mathrm{fr}}$, and $T_{\mathrm{ft}}$ are the time of reverse wave of receiver, transmitter, and the time of forward wave of receiver, transmitter as explained earlier chapter [27]. And since noise in measured TOA can be approximately modeled as Gaussian noise, time of arrival can be calculated as

$$
\tau=t_{t}+n=\frac{d}{v_{p}}+\mu_{t}+n
$$

where $n$ indicates Gaussian (normal) noise, which has mean $\mu_{t}$ and variance $\sigma^{2}$. From the equation above, distance is easily calculated by $\left(\tau-\mu_{t}\right) \cdot v_{p}$. However, since noise has been added during transmission of RF signal between a transmitter and a receiver, distances $\tilde{d}$ calculated from time of arrival in noisy environment is numerically expressed as below

$$
\begin{aligned}
\tilde{d} & =\left(\tau-\mu_{t}\right) \cdot v_{p}=\left(t_{t}+n-\mu_{t}\right) \cdot v_{p} \\
& =\left(t_{t}-\mu_{t}+n\right) \cdot v_{p}=d_{t}+n \cdot v_{p}
\end{aligned}
$$

where $t_{t}, d_{t}$ denotes the time of flight in noiseless environment and the true distance. As obviously shown in formula above, noise added to distance is additive noise and that additive noise, $n \cdot v_{p}$, is still Gaussian noise since $n$ is noise of Gaussian distribution and the speed of light $v_{p}$ is fixed value, which is $3 \cdot 10^{8}$. 


\subsection{Analysis of simulation of RSS with varying sen- sor density}

This section observes root mean squared error (RMSE) of all algorithms introduced in chapter 2 based on received signal strength (RSS) data in three different grid patterns of anchor nodes where the density of anchor sensor nodes varied. Its aim is to observe how the density of anchor nodes affects RMSE, which is used as a barometer showing how accurate the estimation is. The result and the analysis of the simulation is given in following order.

First, non-cooperative least squares estimation and joint map estimation result are shown and analyzed. After that, cooperative least squares estimation is given and the result and its reason is discussed. Last, all of results of estimation with Cramér-Rao lower bound is provided in order to provide the overview of the accuracy of all three algorithms. Before dealing with the results of estimations, setup of this RSS based localization is shortly illustrated to help grasp how the simulation is proceeded. The parameters for basic setup such as a number of transmitting signal are as

\begin{tabular}{|l|c|}
\hline \multicolumn{1}{|c|}{ Parameter } & Value \\
\hline A signal transmitted for each unknown nodes' array & 50 \\
\hline Number of unknown node & 5 \\
\hline Number of different unknown nodes' array & 5 \\
\hline Scope of density of sensor & $10^{-2.0}: 10^{-0.2}: 10^{-2.8}$ \\
\hline Transmission range & $60 \mathrm{~m}$ \\
\hline
\end{tabular}

Table 3.3: Parameter values for RSS with varying a sensor density

As written in table 3.3, each sensor node transmits a signal 50 times to other nodes deployed within transmission range, $60 \mathrm{~m}$ in this simulation. After that, the location of unknown sensor nodes is reformulated and each sensor node again sends a signal 50 times as done for first array of unknown nodes. This procedure is repeated up to the 5 th formulation of unknown sensor nodes. Consequently 250, 50 (the number of signal transmission) $\times 5$ (the number of unknown nodes' formulation), of root mean squared errors (RMSE) are collected at first sensor density.

After that, the density of anchor nodes, a number of anchor nodes per $m^{2}$, is changed by $10^{0.2}$, approximately 0.63 sensor node per $\mathrm{m}^{2}$. And then, 250 of root mean squared error (RMSE) is again computed by going through same procedure as it was for first density of anchor nodes and then, the density of anchor nodes is again changed by $10^{0.2}$ until the density reaches to $10^{-2.8}$.

In figure 3.3, root mean squared error (RMSE) of non-cooperative least squares estimation are given. The blue box in the graph indicates the RMSE which are between 25 and 75 percentile, and the red horizontal bar inside the blue box denotes the median value of 250 RMSE values. In addition, the edge of tail denotes the extreme RMSE value of RMSE set. Red crosses located far from median is 


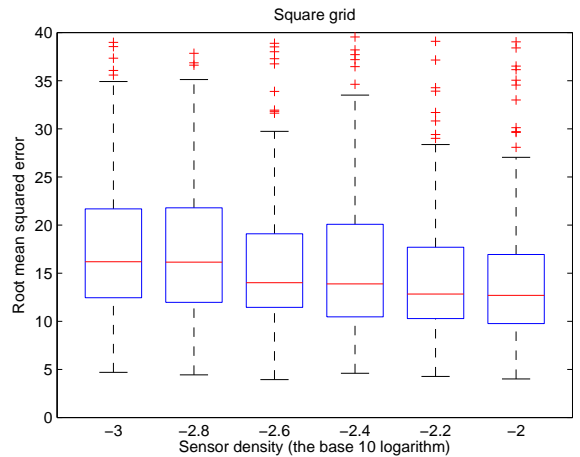

(a) Square grid

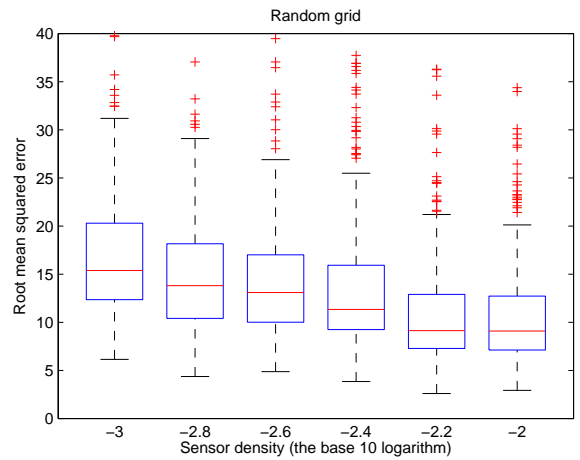

(b) Random grid

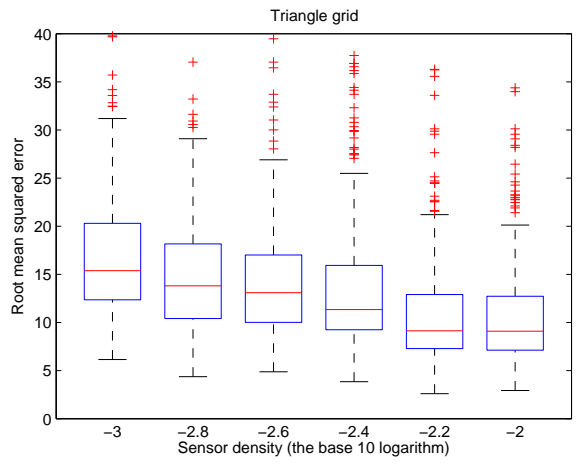

(c) Triangle grid

Figure 3.3: Non-cooperative least squares estimation based on RSS

the outlier. It is observed that median of RMSE decreases as the sensor density of anchor nodes increases in all three grid patterns. In other words, the more anchor sensors present, the higher accuracy is. And among three grid patterns, localization of anchor node in random grid pattern seems to perform best.

However, the performance of random patterned localization may not be consistent as it does not have any particular pattern. And in comparison between square grid and triangle grid, triangle grid patterned localization has higher accuracy than localization based on square grid pattern . Last, it is easily seen that as the height of the blue box, RMSE values between 25 and 75 percentile, are about equal over all sensor density range in all three grid pattern, variance is consistent over all densities. In other words, the stability of the estimation are almost same regardless of the sensor density level.

In spite of its relatively high accuracy in dense environment the accuracy of joint map estimation in Figure 3.4 the accuracy gets drastically worse as the density becomes lower due to its algorithmic characteristic when ill-posed. It seems that a joint map estimation is suitable for dense sensing environment. Unlike in the least squares estimation case where variance is about to equal over all density ranges, as the sensor density is lower, variance of RMSE values become larger, which indicates 


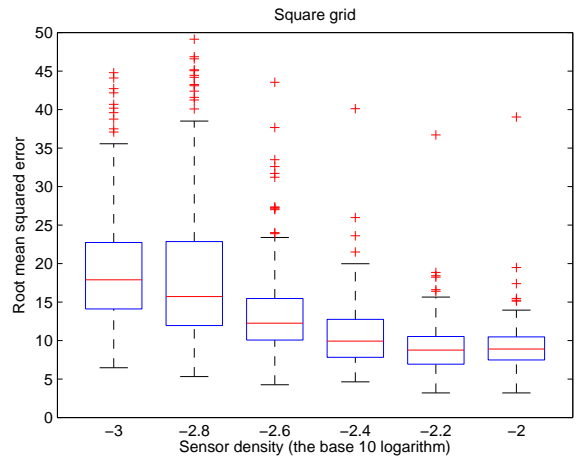

(a) Square grid

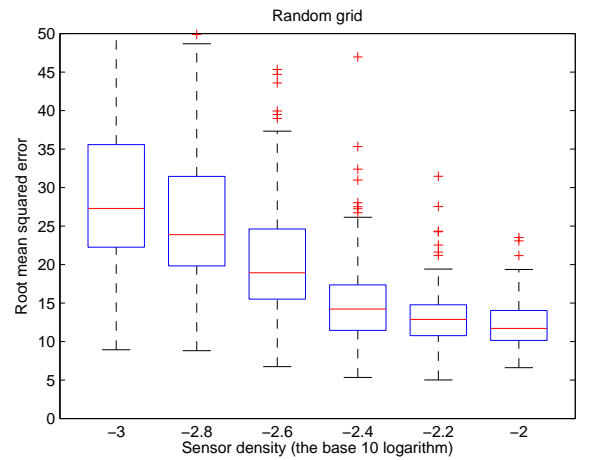

(b) Random grid

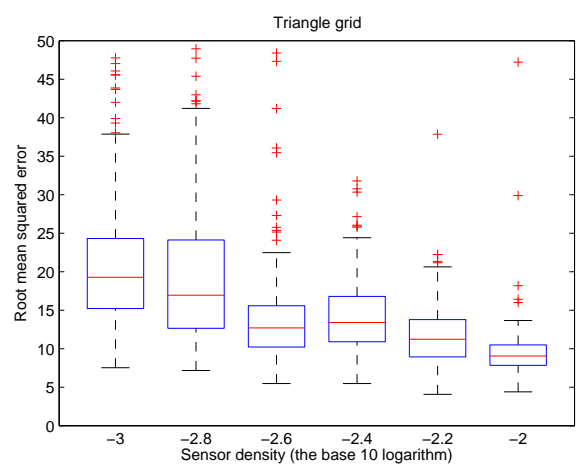

(c) Triangle grid

Figure 3.4: Joint map estimation based on RSS

the location estimation is more unstable. During this simulation, sometimes a very large value of the imaginary part has been computed for location estimate result, and it happens more often when there are more noise or less anchor to localize. For the simulation result, those result has been deemed as the "failed" and not counted in calculating RMSE.

The result of cooperative localization is now given in 3.5, which utilizes measurement data between an unknown node and the anchor nodes as well as between the unknown nodes in estimating objects. Scaled conjugate gradient method has been implemented to compare the accuracy with two non-cooperative localization results which is shown previously. The scaled conjugate gradient is updated the location iteratively, which is operated by function called scg in Matlab.

As expected, a median of RMSE values is much lower than non-cooperative localization algorithms shown before. In addition the height of the blue box indicating general distribution are almost the same over all sensor density range and generally shorter than that in two previous algorithms. That implies that this location estimate is more stable than the other two algorithms and stability level is consistent over all ranges of sensor density. The initial location is estimated by non-cooperative least squares estimation in order to make it easier to converge to the point where 


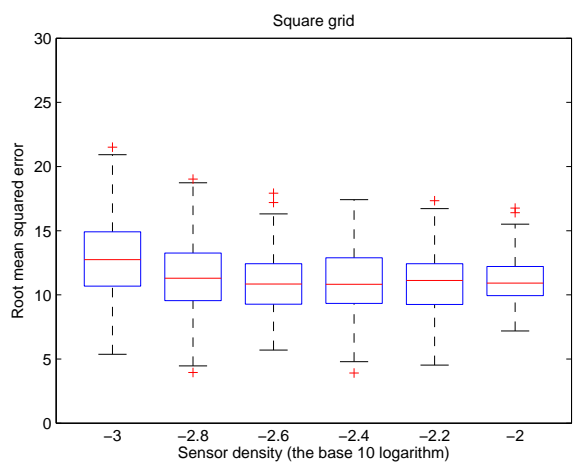

(a) Square grid

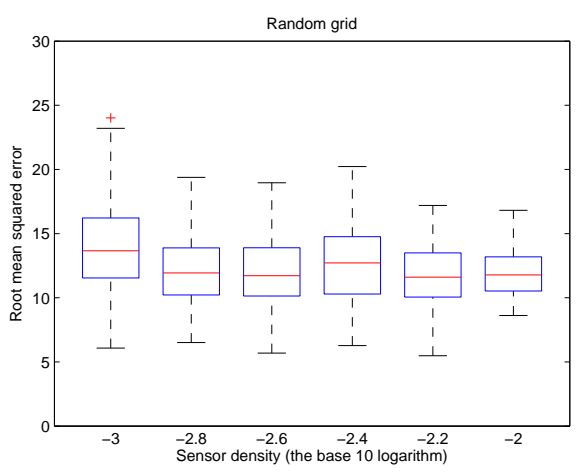

(b) Random grid

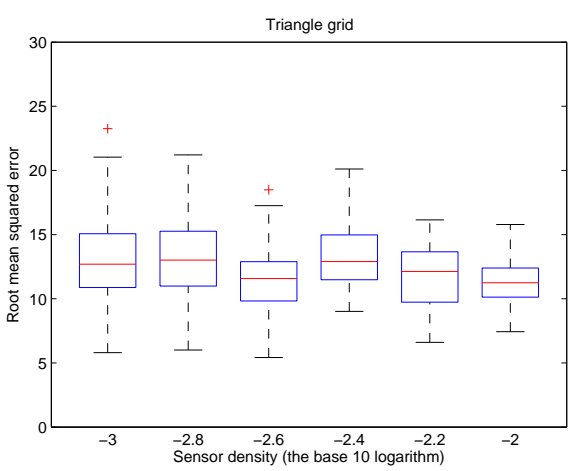

(c) Triangle grid

Figure 3.5: Scaled conjugate gradient based on RSS

the difference between estimated location and true location is minimized.

To give intuition of the general accuracy of all the algorithms is given in
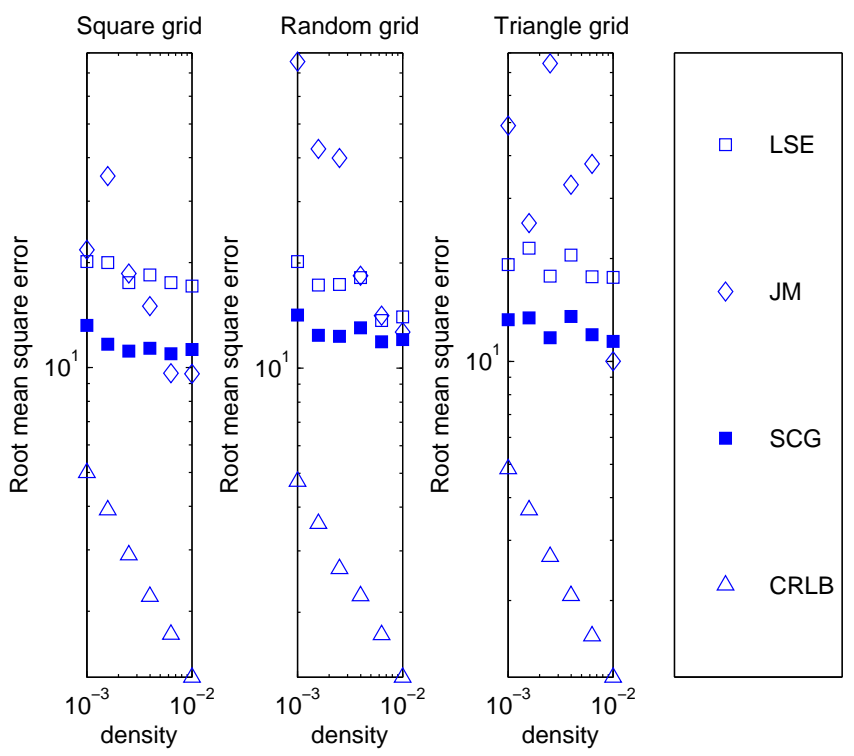

Figure 3.6: All RSS-based localization results with varying density 
Figure 3.6. In this figure, Cramér-Rao lower bound, the lower bound for unbiased estimator, is calculated in order to provide the basic view of how far the algorithms are from the best accuracy possible. The RMSE values above are average RMSE of signal transmitted 50 times with varying unknown nodes' array as written in equation 3.4. LSE, JM, SCG, and CRLB in the figure denote least squares estimation, joint map, scaled conjugate grident, and Cramér-Rao lower bound.It is noticeable that joint map estimation shows even better accuracy than conjugate gradient, cooperative method, at dense environment. But its accuracy becomes worst at less dense scenario. Therefore, joint map estimation is well-suited for the place where many anchor nodes are deployed. And in both least squares and joint map method, the denser the sensing is the smaller RMSE is whereas in the conjugate gradient RMSE is more or less same throughout all the range of sensor density. However, RMSE of all localization algorithms are far above Cramér-Rao lower bound.

\subsection{Analysis of simulation of RSS with varying trans- mission range}

This section examines the accuracy of the localization as a transmission range of a signal is changed so as to learn the relationship between the accuracy and the transmission range under certain sensor node density. Therefore, the aim for this simulation is to observe how well the sensor nodes with limited power, which is more realistic than the previous simulation, can estimate a location of agent nodes and which algorithm is more useful in this scenario. The values of parameters below are used for basic set-up of the simulation in this section.

\begin{tabular}{|l|c|}
\hline \multicolumn{1}{|c|}{ Parameter } & Value \\
\hline A signal transmitted for each unknown nodes' array & 50 \\
\hline Number of unknown node & 10 \\
\hline Times of agent node array change & 5 \\
\hline Scope of density of sensor & $10^{-1.5}$ \\
\hline Transmission range & $30: 20: 90 \mathrm{~m}$ and $\infty$ \\
\hline Standard deviation & $3.4 \mathrm{dBm}$ \\
\hline
\end{tabular}

Table 3.4: Parameter values for RSS with varying a transmission range

As shown in table 3.4, the set-up for this simulation is very similar to the simulation in the previous section. But in this section, the density of sensor node is fixed by $10^{-1.5}$ (approximately 0.03 sensor node per $\mathrm{m}^{2}$ ) with a transmission range of sensor nodes varying (from $30 \mathrm{~m}$ to $90 \mathrm{~m}$ with $20 \mathrm{~m}$ interval). Since each sensor node transmits a signal 50 times to one another in five different unknown node formulations, a number of accumulated root mean squared errors at each transmission range is $250,50 \cdot 5$ (a number of a signal transmitted $\times$ a number of a different formulation of unknown nodes). And a localization with unlimited range has also been made to compare the localization result with long range such as $90 \mathrm{~m}$ or even longer distance. As done in previous section, two non-cooperative localization algo- 
rithms are introduced first and scaled conjugate gradient, cooperative localization algorithm, is followed.

As before, the initial location to be updated in scaled conjugate gradient is the estimated location by least squares estimation. And for the rest scaled conjugate gradients, the initial point are set in the same way.

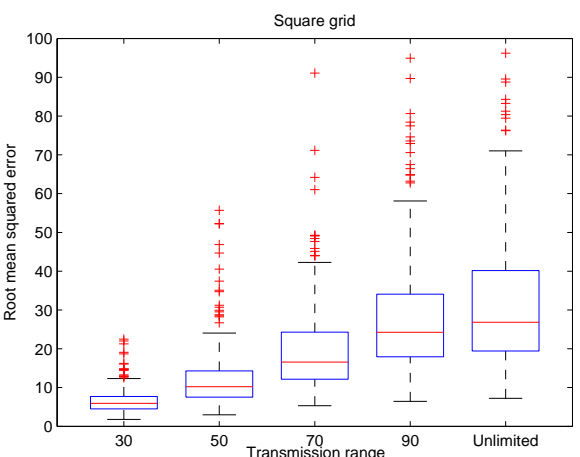

(a) Square grid

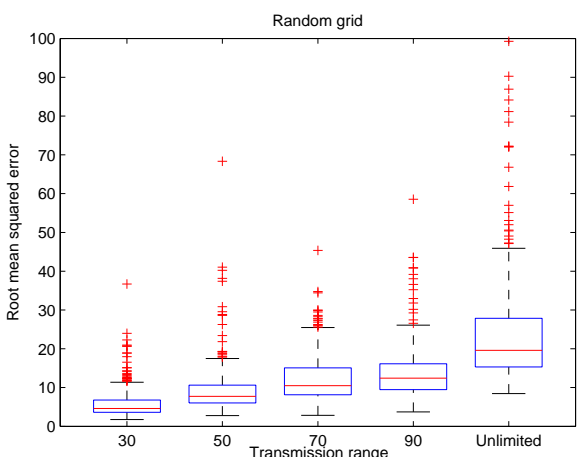

(b) Random grid

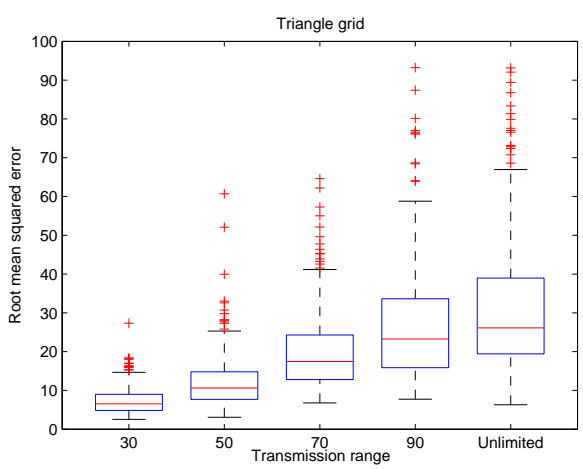

(c) Triangle grid

Figure 3.7: Linear least Squares estimation based on RSS

In figure 3.7, the median of 250 RMSE values of 10 unknown nodes estimated by least squares algorithm are increased as the transmission range is longer. Since longer transmission range involves more sensor data to estimate agent nodes, longer transmission range scenario was expected to come up with better accuracy as proven in [18]. However, surprisingly as transmission range is longer, the accuracy gets worse, which was expected to happen in the opposite way. That is because received signal power from RSS is affected by log-normal shadowing, the relationship between the received power and distance is nonlinear. As shown in section 3.1.2, the received power and the distance can be expressed as:

$$
P_{r}=P_{r}\left(d_{o}\right)-\log _{10} d+n
$$

where $P_{r}, P_{0}, d, n$ is the received power, received signal power at reference distance, the distance between a transmitter and a receiver, and Gaussian noise respectively. 
Since the distance estimated $\tilde{d}$ is calculated from $\left.P_{r}=P_{0}\right)-\log _{10} \tilde{d}$, the difference between actual distance and estimated distance can be expressed as $d \cdot\left(10^{n}-1\right)$ showing that distance estimated from received power has non-linear relationship with respect to the true distance $d$.

Therefore, as the distance between the transmitter and the receiver becomes longer, the signal strength gets drastically weaker due to the its non-linear relationship with distance, which leads to less accurate estimation in the algorithm suitable for linear model. Therefore, the linear least squares estimation, solution of linear system, shows poorer accuracy as transmitting distance is increased meaning since received power being exponentially decreased is more likely to be involved in estimating a location. In addition, it can be easily seen that the localization with the longer transmission range also causes lager variance since the height of 25 to 75 percentile box becomes taller as the transmission range is longer. In terms of accuracy, all three grid patterned localization results seemed similar to one another.

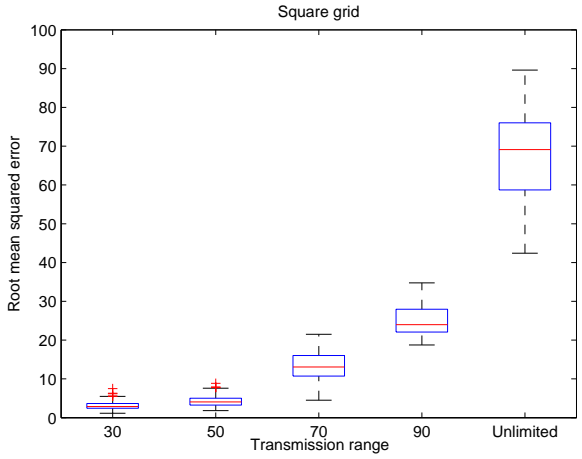

(a) Square grid

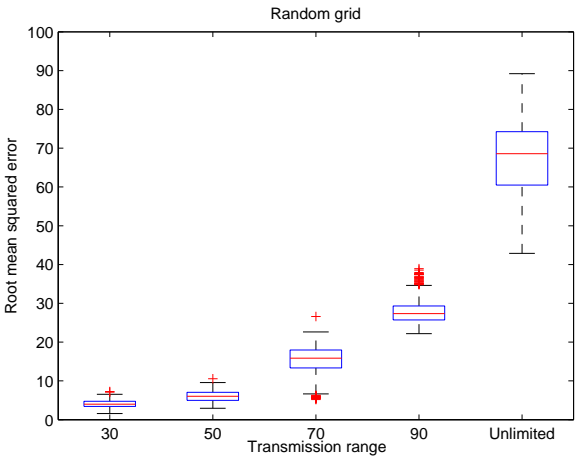

(b) Random grid

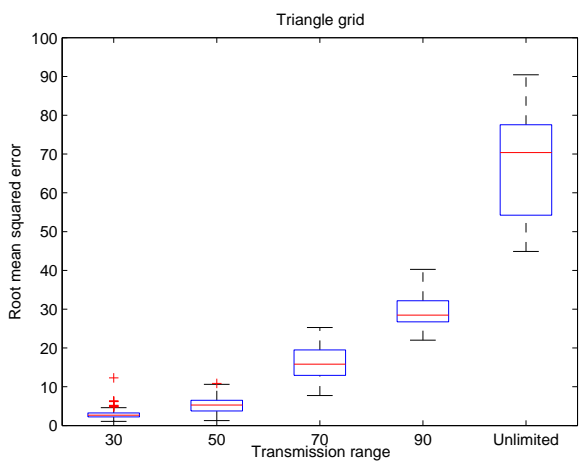

(c) Triangle grid

Figure 3.8: Joint map estimation based on RSS

The result of joint map estimation in Figure 3.8 shows its characteristic clearly. For localization with short transmission range $(30 \mathrm{~m}$ and $50 \mathrm{~m})$ where only the received signals which has small noise, it displays relatively high accuracy whereas its accuracy gets drastically worse as the signals received from long distance are involved 
in location estimate just like least squares estimation. In addition, as the height of percentile box proves the longer the transmission distance involved in localization is, the more unstable the estimation is. This result looks very similar to the result of the joint map estimation in the environment where the sensor density varies. In terms of stability and accuracy, the joint map algorithm shows high sensitivity on noise.

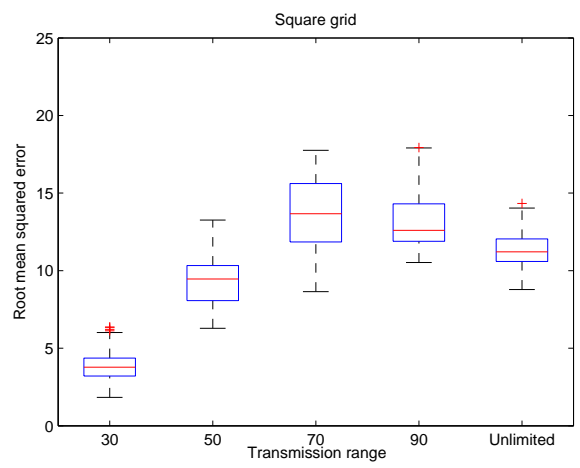

(a) Square grid

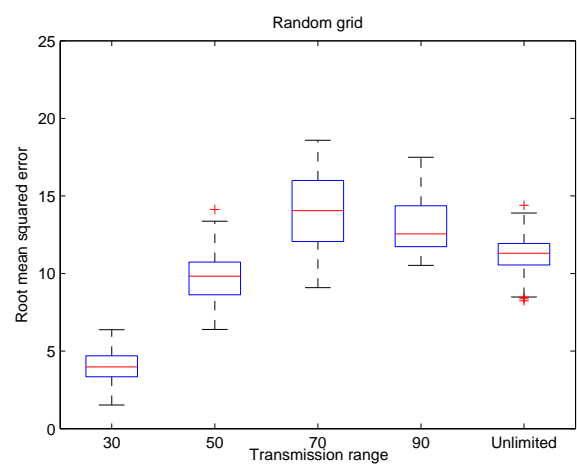

(b) Random grid

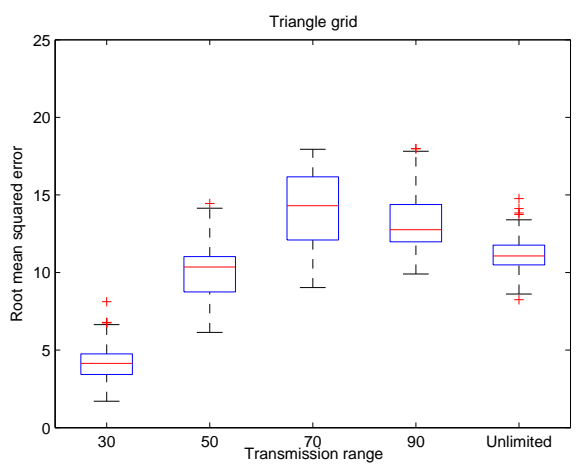

(c) Triangle grid

Figure 3.9: Scaled conjugate gradient based on RSS

In Figure 3.9, localization result of the conjugate gradient, the medians of RMSE throughout all the range of transmission are generally lower than two previous localization in all three grid patterns as shown. The attractive result in this localization is the transmission range that gives highest RMSE median and highest error variance in all three grid pattern is $70 \mathrm{~m}$ unlike previous two localizations where unlimited transmission range based localization had worst performance.

Furthermore, the median in unlimited range is even lower than that in $70 \mathrm{~m}$. It might be because when transmission range is longer than $70 \mathrm{~m}$ a number of sensors involved in localization is large enough to overcome high noise. And in aspect of performance of each grid pattern, all grid patterned localizations show fairly the same result in both accuracy and stability.

From the figure 3.10 the average RMSE with transmission range of $30 \mathrm{~m}$ up to $90 \mathrm{~m}$ of all algorithms including Cramér-Rao lower bound can be observed as the 


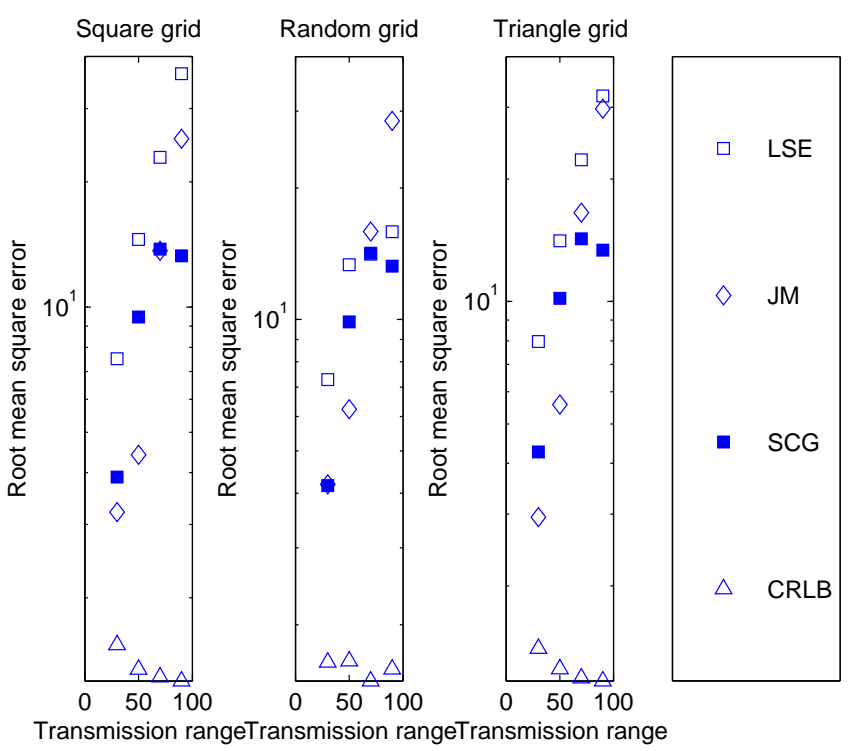

Figure 3.10: All RSS-based localization results with varying transmission range

transmission range is increased up to $90 \mathrm{~m}$.

It is easily seen that cooperative localization using conjugate gradient, generally outperforms other non-cooperative localizations (least squares estimation and joint map estimation) except for when transmission range is $30 \mathrm{~m}$ and $50 \mathrm{~m}$. And average RMSE of both least squares estimation and joint map estimation gets higher as transmission range is longer meanwhile conjugate gradient shows better accuracy for $70 m$ transmission range. Last, it is noticeable that median of RMSE and average RMSE is more or less the same.

\subsection{Analysis of simulation of TOA with varying the sensor density}

This section conducts a localization based on time of arrival (TOA) data with varying a sensor density. Same localization algorithms and grid patterns used for the RSS based simulation are again implemented in this simulation in order to see how different the localization results under identical conditions are between RSS and TOA based localization. The table 3.2 shows the parameter used for setup of TOA based simulation in this section.

The way of collecting RMSE values at each sensor density is basically same as that in RSS based simulation with varying a sensor node. However, in this simulation a number of signal transmitted is 100 with 10 different unknown nodes' array, which ends up with collecting 1000 RMSE at each density. And the number of unknown nodes is 10, which is twice of that in RSS based localization. Noncooperative localizations' results is given first. After that, cooperative localization (conjugate gradient) result is followed. Last, average RMSE of all the algorithms are displayed to give overall intuition in terms of the accuracy as done in RSS based 


\begin{tabular}{|l|c|}
\hline \multicolumn{1}{|c|}{ Parameter } & Value \\
\hline A signal transmitted for each unknown nodes' array & 100 \\
\hline Number of unknown node & 10 \\
\hline Times of agent node array change & 10 \\
\hline Transmission range & $60 \mathrm{~m}$ \\
\hline Density of a sensor & $10^{-2:-0.2:-3} \mathrm{~m}^{2}$ \\
\hline
\end{tabular}

Table 3.5: Parameter values for TOA with varying a sensor density localization.

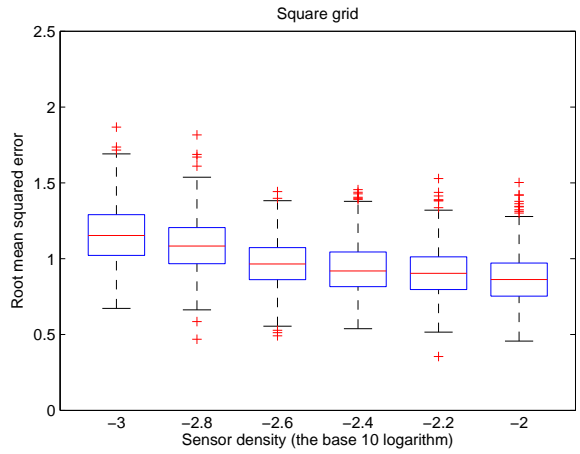

(a) Square grid

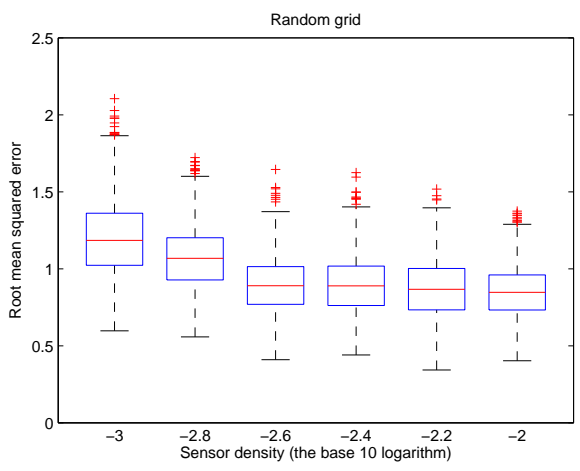

(b) Random grid

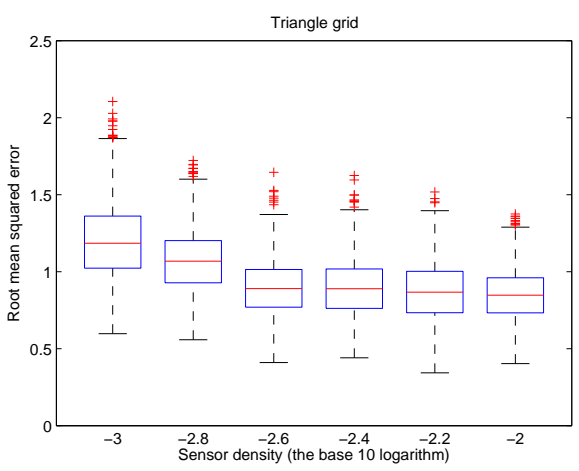

(c) Triangle grid

Figure 3.11: Linear least Squares estimation based on TOA

As expected, in Figure 3.11 the linear least squares estimation (LSE) shows very good accuracy in TOA based localization since the noise is additive, which leads to the linear relation between distance estimated from TOA data and the actual distance in contrast with non-linear relationship between estimated distance from RSS data and the actual distance. To explain mathematically, $d_{n}=d_{t}+n \cdot v_{p}$ where $d_{n}, d_{t}, n, v_{p}$ denotes distance measured, true distance, noise, and propagation velocity respectively as shown in Equation 3.14. And since $n$ is Gaussian noise and $v_{p}$ is fixed value, the distance calculated from TOA data is linearly related to the actual distance. 
The localization results from all the grid patterned anchor nodes are similar to one another. The height of blue box representing half, $25 \% \sim 75 \%$, of RMSE values closest to the median is more or less same over all the range of sensor density, which is meaning that variance (stability) is consistent. It is noticeable that the median of RMSE decreases as a sensor becomes more dense.

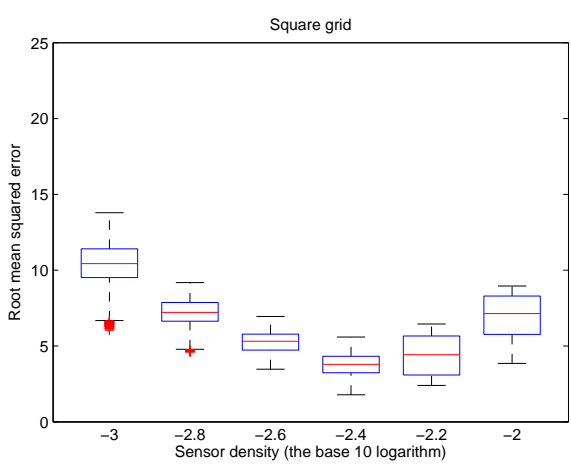

(a) Square grid

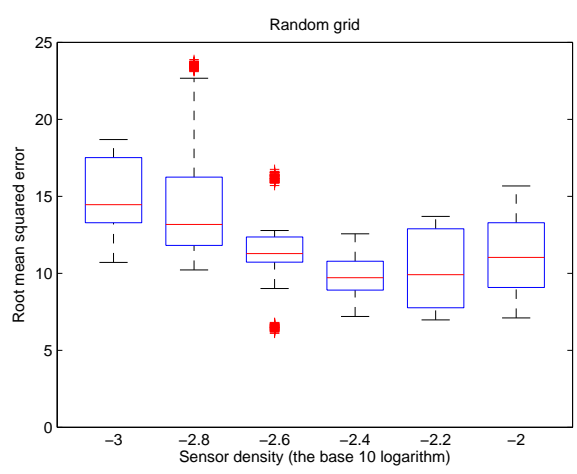

(b) Random grid

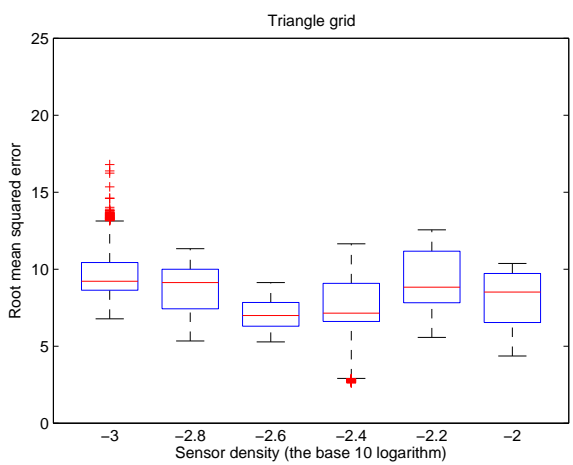

(c) Triangle grid

Figure 3.12: Joint map estimation based on TOA

In Figure 3.12, localization at higher sensor density does not guarantee higher accuracy in joint map estimation unlike the linear least squares estimation just shown previously. Instead, it seemed the accuracy is best at density $10^{-2.4}$ per $\mathrm{m}^{2}$ in all grid pattern since median of RMSE values is lowest at that density in all grid patterns. And among all three grid patterns, since square grid pattern shows the lowest median and the blue box in square grid has shorter height throughout all sensor density than the other two grid patterns, the localization based on square grid patterned anchor nodes can be said to outperform other grid patterned estimations in terms of stability and accuracy.

Compared to least squares estimation the joint map estimation's result is much worse in aspect of accuracy in all the range of sensor density. And the stability is pretty inconsistent as the height of blue box is variant over all ranges of sensor density. In other words, the linear least squares estimation is more optimized in estimating location from the data where additive noise is present than joint map estimation. 


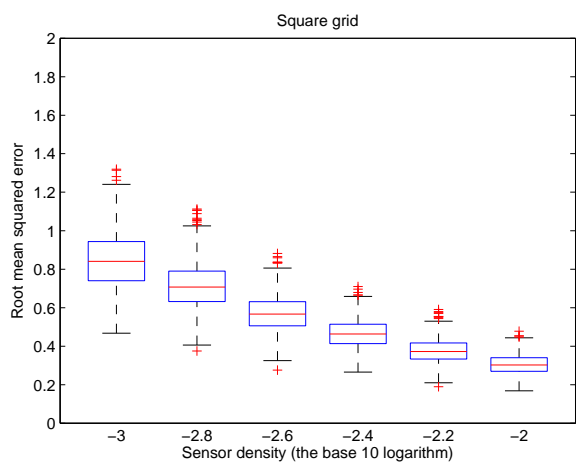

(a) Square grid

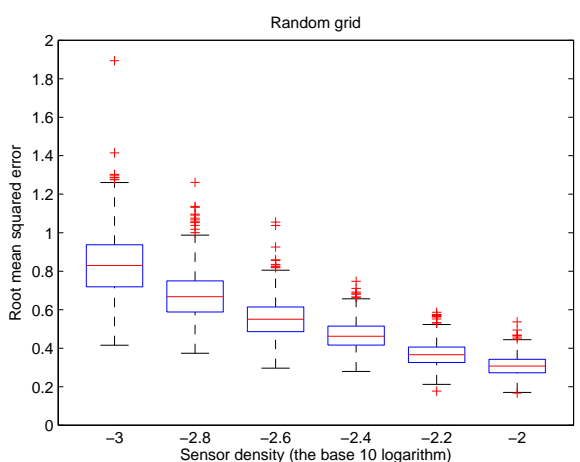

(b) Random grid

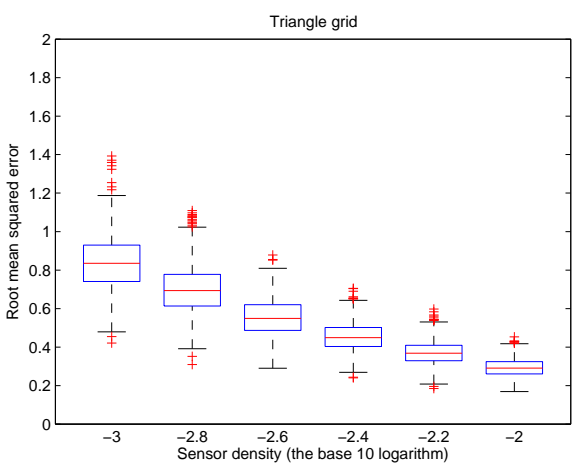

(c) Triangle grid

Figure 3.13: Scaled conjugate gradient based on TOA

The result of conjugate gradient is now observed in order to prove that cooperative localization algorithm outperforms non-cooperative method (linear squares estimation, joint map estimation). In Figure 3.13, it is clear that the median of RMSE of conjugate gradient is lower than the previous two non-cooperative localization algorithms in all grid pattern. Since the result of the grid pattern is similar to one another it is hard to state which grid patterned localization performs best.

It is important to note that as sensor density gets higher the accuracy is better in all of grid patterns as observed in linear squares estimation. In addition, the higher the density of the sensor the shorter the percentile box becomes. And even extreme RMSE value, the value which is neither between 25 and 75 percentile nor outlier, becomes farther from median when a sensor density is reduced. In other words, as the sensor becomes less dense the localization estimation becomes more unstable even though its tallest percentile box is still shorter or equal to the shortest one in the least squares estimation.

The figure 3.14, average RMSE calculated as expressed in the Equation 3.1.2 gives more clear overview of the accuracy of all three different algorithms in all grid patterns. In addition, the Cramér-Rao lower bound is also shown to give an idea how well localization has been done and how much the accuracy of the algorithms implemented needed to be better. As we already expected from the median in Figures $3.11 \sim 3.13$, the average RMSE of the joint map estimation is highest, meaning 

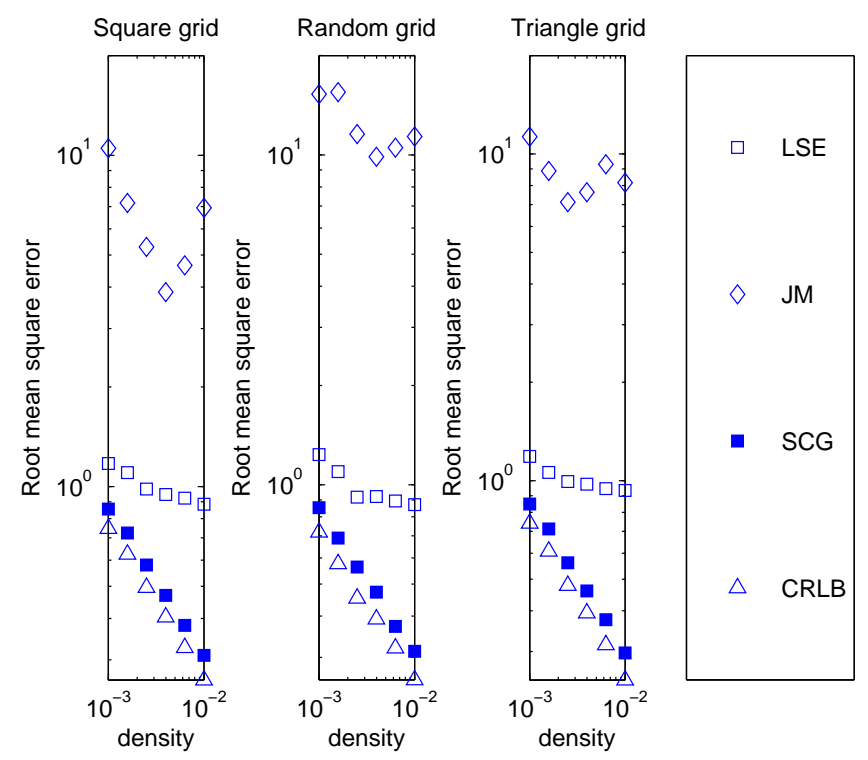

Figure 3.14: All TOA-based localization results with varying density

of most inaccurate among all three algorithms. And among the grid patterns in the joint map method the squared gird patterned localization shows best accuracy as it has obviously seen in the figure above. But for rest two grid patterns it is hard to tell which pattern has higher accuracy.

It can be seen that conjugate gradient estimation is clearly more accurate than the least squares. In aspect of performance of cooperative method, the conjugate gradient based location estimate almost reaches to Cramér-Rao lower bound in a dense sensory environment not like in the RSS case. And it is easy to observe that as the sensor density is higher, average RMSE of localization using the conjugate gradient is closer to Cramér-Rao lower bound.

\subsection{Analysis of simulation of TOA with varying trans- mission range}

This section provides the localization result of the algorithm with varying transmission range so as to observe the influence of transmission range based on TOA data. The aim of the simulation in this section is to see the influence on RMSE for both cooperative and non-cooperative algorithms as a signal transmission range varies. Most of setup for this simulation such as a number of unknown nodes and step size and so on are same as TOA simulation implemented in previous section. To give a basic information about the parameter of basic setting regrading simulation is first introduced. After that, for the result of a localization algorithm, the linear least squares estimation, the joint map estimation, and the conjugate gradient estimation are given in the order.

As shown in Table 3.6 the simulation setup is similar to RSS based simulation 


\begin{tabular}{|l|c|}
\hline \multicolumn{1}{|c|}{ Parameter } & Value \\
\hline A signal transmitted for each unknown nodes' array & 50 \\
\hline Number of unknown node & 5 \\
\hline Times of agent node array change & 10 \\
\hline Transmission range & $20 m: 10 \mathrm{~m}: 100 \mathrm{~m}$ and $\infty$ \\
\hline Density of a sensor & $10^{-1.5} \mathrm{~m}^{2}$ \\
\hline
\end{tabular}

Table 3.6: Parameter values for TOA with varying a transmission range

introduced previously. A number of signal transmitted from each sensor node is 50 with 10 different formulations of unknown nodes, resulting in 500 RMSE accumulated at each transmission range. As before, based on a median of RMSE throughout all the range of transmission the accuracy is observed in order to figure out how the transmission range affects the accuracy under all three grid patterned localization with each of algorithm mentioned over this chapter.

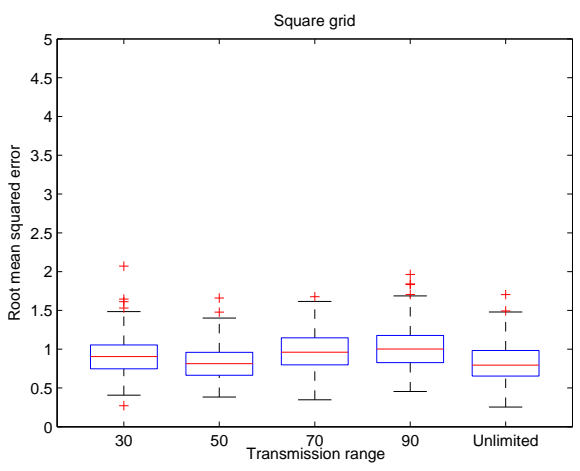

(a) Square grid

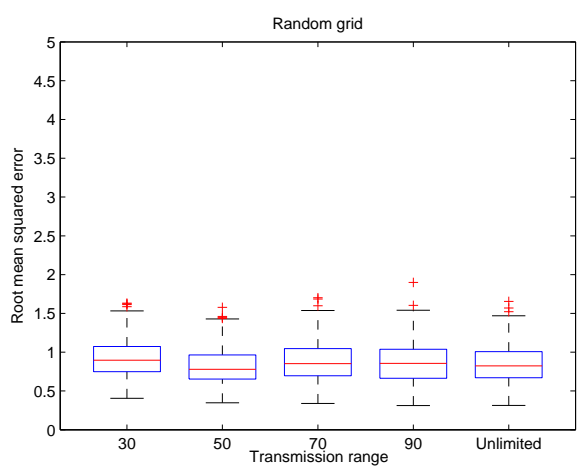

(b) Random grid

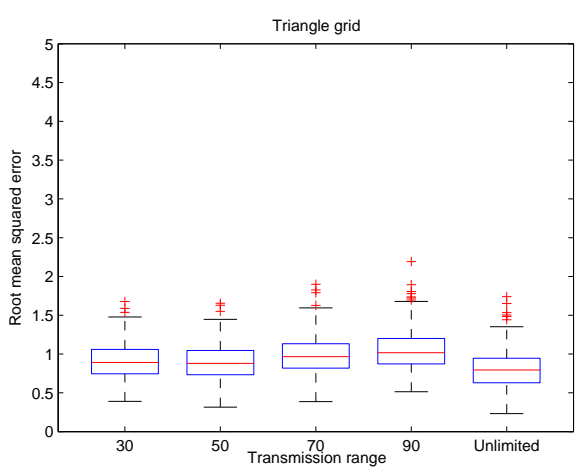

(c) Triangle grid

Figure 3.15: Linear least Squares estimation based on TOA

Figure 3.15 shows the median of RMSE values of linear least squares estimation has a very low value, which indicates high accuracy. And longer transmission range does not affect the difference between estimated distance and true distance anymore 
since the noise type present in TOA case is additive. In other words, the transmission range does not affect the accuracy in TOA based location estimate.

However, in the unlimited range case, where involves most sensor in localizing among all the transmission range presented in the simulation, has best accuracy, involves most sensors in localization among all transmission ranges presented in the simulation.

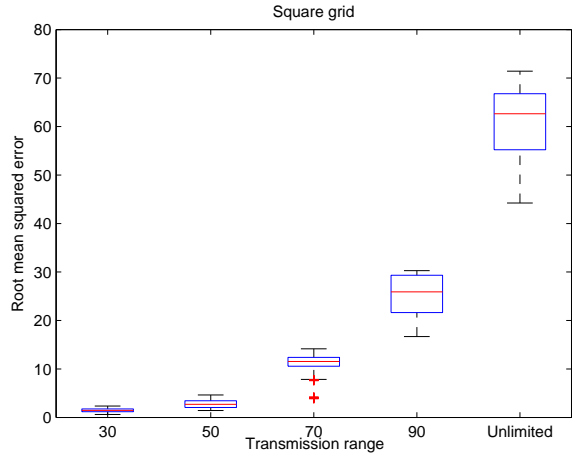

(a) Square grid

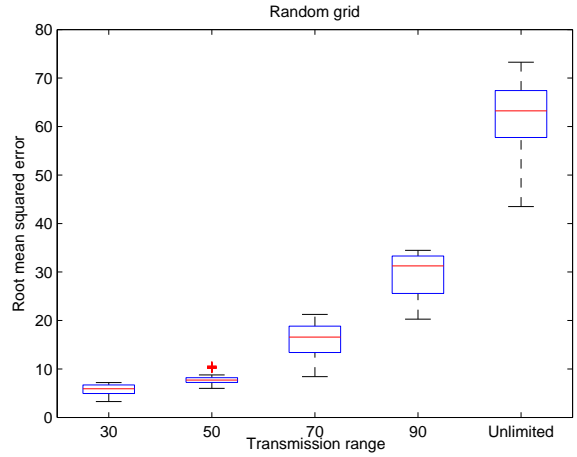

(b) Random grid

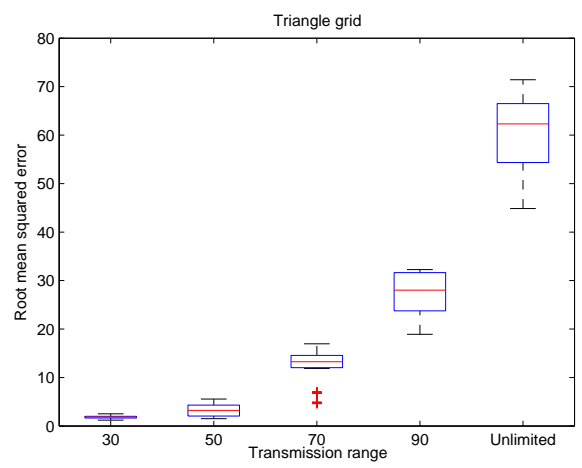

(c) Triangle grid

Figure 3.16: Joint map estimation based on TOA

On the other hand, it seemed like regardless of type of noise, joint map estimation performs poorly as a transmission range is longer as clearly shown in figure 3.16. In all grid patterns, as transmission range is longer the accuracy gets worse. Furthermore, the height of blue box becomes higher, meaning that the estimation is more unstable and stability is not consistent. However, in the shortest range $(30 \mathrm{~m})$, the estimation is accurate and has stable estimation as seen from short percentile box. Therefore, joint map estimation can be said to be not suitable algorithm in localization from long distance like $50 \mathrm{~m}$ or longer.

In Figure 3.17, the localization results of the scaled conjugate gradient can be observed. As it is obviously seen, RMSE of the scaled conjugate gradient is lower than that estimated by non-cooperative LSE methods. And as a transmission distance is longer the accuracy becomes higher in all grid patterns. That is because localization in longer transmission range involves more sensors in estimating a loca- 


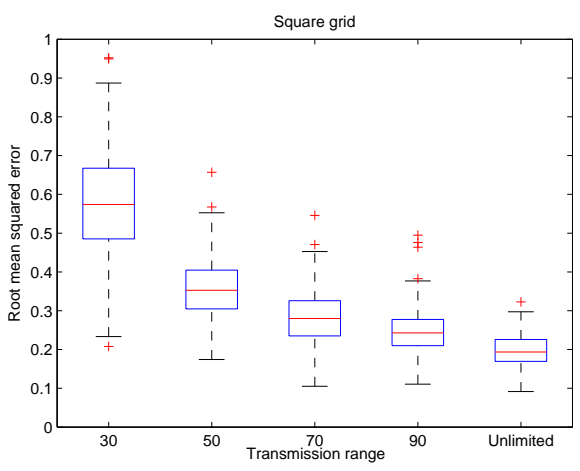

(a) Square grid

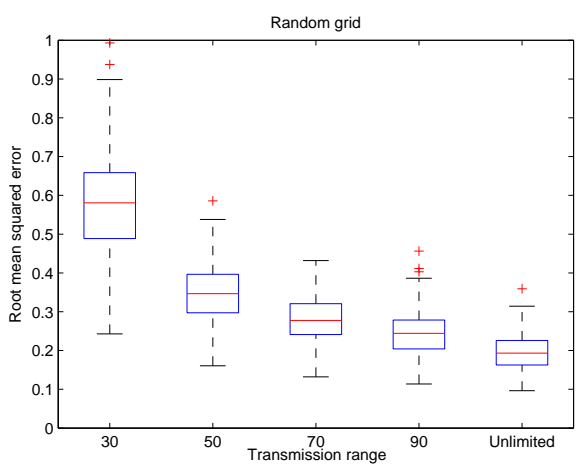

(b) Random grid

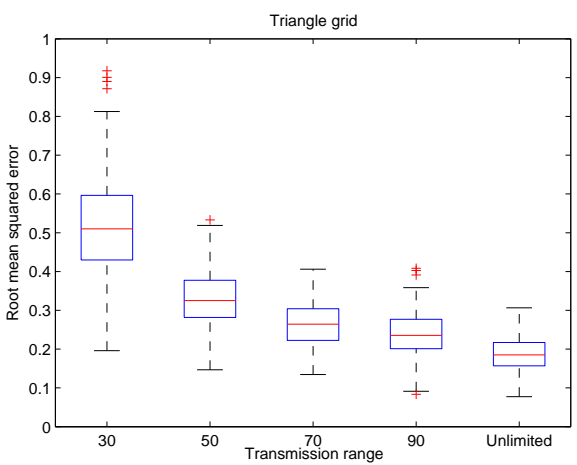

(c) Triangle grid

Figure 3.17: Scaled conjugate gradient based on TOA

tion and a distant localization based on TOA data does not degrade an accuracy in conjugate gradient algorithm.

Therefore, the result here is very similar to the case when the sensor density is varied. Furthermore, the distribution of half of 500 RMSE values collected closest to the median is narrower as the transmission range is longer, which indicates that the stability of the estimation gets better. As a result, localization in unlimited transmission case showed the best accuracy where the median of RMSE is just about 0.2 and the distribution of RMSE values between 25 and 75 percentile about median is very narrow.

Figure 3.18 shows the average RMSE with transmission range of $30 \mathrm{~m}$ up to $90 \mathrm{~m}$ in all three grid patterns of all algorithms to provide general view of the accuracy of all algorithm. From this figure high accuracy of both non cooperative and cooperative least squares estimation can be observed whereas joint map estimation suffers accuracy especially at long transmission range. In the case of joint map estimation, despite of its poor accuracy, it is meaningful to know that anchor nodes in a square grid pattern always performs best among all three grid patterns for joint map estimation.

Cooperative least squares method (conjugate gradient) is again clearly more accurate than non-cooperative least squares estimation. As expected from the previous result of conjugate gradient, average RMSE of conjugate gradient almost reaches 


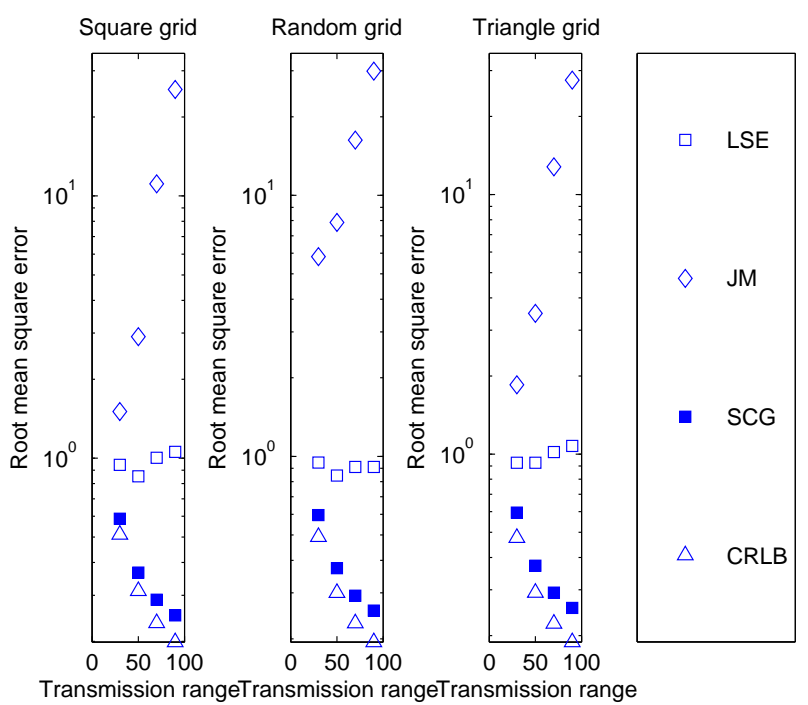

Figure 3.18: All TOA-based localization results with varying transmission range the Cramér-Rao lower bound at long transmission distance. 


\section{Chapter 4}

\section{Analysis of measured data and comparison to simulated data}

This chapter compares the result of simulated data based localization to measured data based localization. First of all, the localization campaign based on received signal strength (RSS) and time of arrival (TOA) conducted by some researcher is introduced with relevant informations such as the place of experiment, method use for data measured, and so on. After that, both RSS and TOA data are simulated using same parameter like a path loss exponent and a standard deviation as used in the experiment so that simulated data based localization and measured data based localization can be fairly compared to each other. Lastly, the simulations implemented are analyzed and evaluated.

\subsection{Basic information about measurement}

RSS and TOA measurement data are collected by Neal Patwari, assistant professor at University of Utah, supported by Motorola labs' Florida communications research lab, in Plantation, Florida [40]. For this experiment, 44 sensors are deployed in the office $(14 \mathrm{~m}$ $\times 13 m$ ) as shown in Figure 4.1.

The campaign is performed as follows. First, the transmitter is placed at the location $\# 1$ while changing the location of receiver from $\# 2$ to $\# 44$. Between the transmitter and each receiver, signals are transmitted five times. After the transmitter at the location $\# 1$

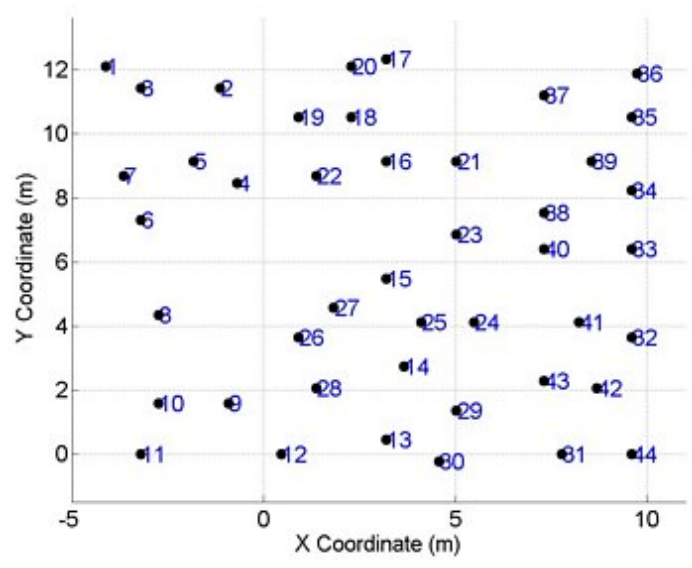

Figure 4.1: The position of sensor nodes deployed at research lab transmitted a signal five times up to the 
receiver placed at $\# 44$, the transmitter is then relocated to the location $\# 2$ and transmits signals to receiver at $\# 1$ and at $\# 3$ through $\# 44$ five times again. This procedure is continued until the transmitter is placed at the location \#44. Consequently, there are 10 times measurement made (five times as a transmitter and another five times as a receiver) for any pair out of 44 locations drawn in Figure 4.1. That is how the measurement was conducted. And the measurement data from each pair of 44 locations is the average of 10times measurement. In this campaign both time of arrival (TOA) and received signal strength (RSS) are measured.

The sensor device uses a wide band direct-sequence spread-spectrum (DS-SS) transceiver (Sigtek model ST-515) [37]. The specification of the device is in Table 4.1 .

\begin{tabular}{|l|c|}
\hline \multicolumn{1}{|c|}{ Parameter } & Value \\
\hline Chip rate & $40 \mathrm{MHz}$ \\
\hline Code length & 1024 \\
\hline Center frequency & $2443 \mathrm{MHz}$ \\
\hline Transmit power & $10 \mathrm{~mW}$ \\
\hline Antenna & $2.4 \mathrm{GHz}$ sleeve dipole antenna \\
\hline Measured gain of antenna & $1.1 \mathrm{dBi}$ \\
\hline
\end{tabular}

Table 4.1: The specification of a sensor node

Throughout the experiment SNR had been kept over $25 \mathrm{~dB}$ to avoid noise and ISM-band interference issue. For TOA measurement, a one-way measurement method was chosen and time-synchronization tool has been equipped to synchronize a time clock between a receiver and a transmitter [37]. In the following sections, information related to measurement data of RSS and TOA will be briefly introduced. Measured data based localization and simulated data based localization will be conducted. Then, both simulated data and measured data will be utilized in estimating location of unknown nodes using the localization algorithms mentioned in chapter2. And finally the results of measured data based localization and simulated data based one will be analyzed and compared to each other.

\subsection{Comparison for RSS based localization}

In this section, the information of RSS data measured from the campaign given the in previous chapter is provided first. And the data simulated based on same parameters values of measured data are utilized in localization on the purpose of comparing with localization results made from measured data. Table 4.2 shows fundamental parameter for the measured data to give basic idea how to generate RSS simulated data.

Received power at the reference distance $P_{r}\left(d_{o}\right)$ is computed by $P_{\text {out }}-C_{t}+$ $G_{t}-P_{L}\left(d_{o}\right)+G_{r}-C_{r}(\mathrm{dBm})$, where $P_{\text {out }}$ is output power transmitted, $P_{L}\left(d_{o}\right)$ is the path loss at reference distance $d_{o}$, and $C_{t}, C_{r}, G_{t}, G_{r}$ are attenuation and gain of 


\begin{tabular}{|l|l|c|}
\hline \multicolumn{3}{|c|}{ Parameters and their values of RSS experiment } \\
\hline$P_{r}\left(d_{0}\right)$ & Received power at the reference distance $(1 \mathrm{~m})$ in $\mathrm{dBm}$ & $-37.5 \mathrm{dBm}$ \\
\hline$n_{p}$ & Path loss exponent & 2.3 \\
\hline$\sigma_{\mathrm{dB}}$ & Standard deviation & $3.9 \mathrm{dBm}$ \\
\hline
\end{tabular}

Table 4.2: The measurement data of RSS

cable and antenna as described in Table 3.1. Therefore, received power $P_{r}$ can be mathematically expressed as below.

$$
P_{r}=P_{r}\left(d_{0}\right)-n_{p} \cdot \log _{10} d+n
$$

where $n_{p}, d_{o}$, and $n$ denotes observed path loss exponent and reference distance $(1 \mathrm{~m})$, and noise with normal distribution respectively. Therefore, since $\log _{10} \tilde{d}$ can be replaced to $\log _{10} d+n$, received signal can be again expressed as

$$
P_{r}=P_{r}\left(d_{0}\right)-n_{p} \cdot \log _{10} \tilde{\mathrm{d}}
$$

where $\tilde{d}$ denotes estimated distance from noisy environment. Therefore, $\tilde{d}$ can be calculated from received signal as

$$
\tilde{d}=10^{\frac{P_{r}\left(d_{0}\right)-P_{r}}{n_{p}}}
$$

A received signal power without noise $P_{o}$ is computed as $P_{o}=P_{r}\left(d_{o}\right)-n_{p}$. $\log _{10} d$, where $d$ is true location between anchor node and unknown node. Standard deviation $\sigma$ is therefore calculated from

$$
\sigma=\sqrt{\frac{\sum_{i=1}^{N}\left(P_{o}^{(i)}-P_{r}^{(i)}\right)^{2}}{N}}
$$

where $N, P_{o}^{(i)}$, and $P_{r}^{(i)}$ is the number of a measurement conducted, received power between $i$ th node pair out of 44 nodes in noiseless environment, and the $i$ th node pair's received power in noisy environment. Since there are 44 sensor nodes and measurement is made between a sensor and the rest 43 nodes, a number of measurement data $N$ must be $(43 \times 44) / 2$, which is 946 .

That is how $\sigma_{\mathrm{dB}}$ in the table 4.2 is calculated. Under log-normal shadowing assumption, all same parameter values in table 4.2 and locations of 44 sensors are used for generating simulated data to be able to compare measured data. Using the RSS data simulated, the comparison of localization based on both measured data and simulated data is made with following set-up as given in Table 4.3 below.

\begin{tabular}{|l|c|}
\hline \multicolumn{1}{|c|}{ Parameter } & Value \\
\hline Number of unknown node & 10 \\
\hline Number of anchor node & 34 \\
\hline Times of node shift & 100 \\
\hline
\end{tabular}

Table 4.3: RSS based localization set-up

As noticed in the table above, 10 sensor nodes selected randomly out of 44 sensor nodes are set to be unknown. In other words, 34 anchor sensor nodes with 
10 unknown nodes are present. Since measured data has 3.9 (in $\mathrm{dBm}$ ) as standard deviation of received power, the data is simulated by adding Gaussian noise with standard deviation, 3.9 , on noiseless received signal, $P_{r}\left(d_{0}\right)-n_{p} \cdot \log _{10} d$, which results in log-normal fading over transmitting distance $d$. After average root mean squared error (RMSE) of the localization is calculated for the first unknown nodes' array, the array of unknown nodes is changed. After that, RMSE is computed again. This random unknown node shift is processed up to 100 times. Consequently, every single algorithm to be implemented will have average RMSE from each of 100 different unknown nodes' array.

This section categorizes algorithms into two categories: least squares estimation (LSE) and non-linear least squares estimation. Therefore, the result of two algorithms under least squares category (non-cooperative LSE and cooperative LSE) is given first. And later, the result of two algorithms under non-least squares category (non-cooperative joint map estimation and cooperative joint map estimation) is followed. More detail explanation about cooperative joint map estimation is left to a later section of this chapter.

\subsubsection{Least squares based localization}

This section displays the difference of localization result between non-cooperative LSE and cooperative LSE based on both measured RSS data and simulated RSS data in order to compare not just the localization methods but also the results based on measured data and simulated data.

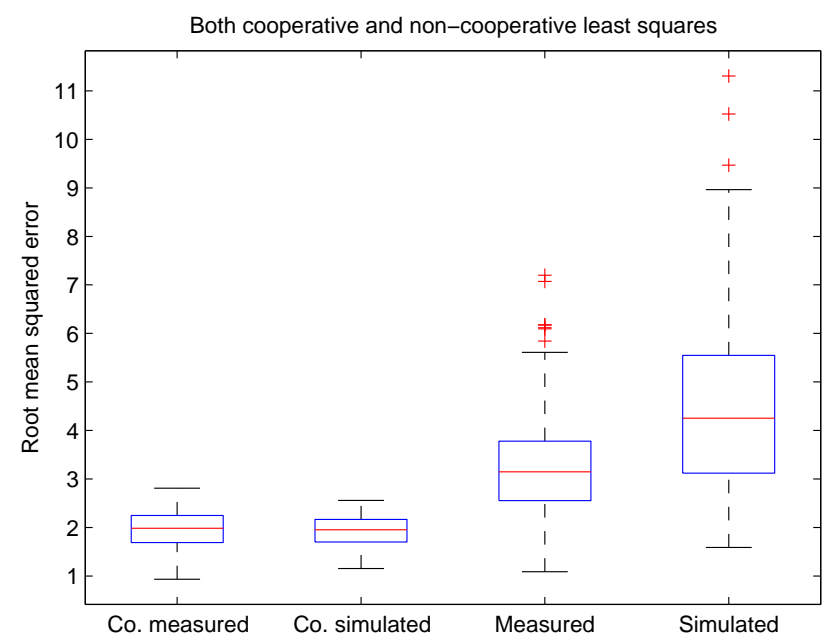

Figure 4.2: RSS based least squares estimation with CRLB

Figure 4.2 shows the result of non-cooperative and cooperative least squares ( scaled conjugate gradient) estimation. As it is obviously seen in Figure 4.2, measured data based location estimate generally more precise than the localization based on simulated data in non-cooperative method. And also percentile box representing value range from 25 to 75 percentile is much shorter than that in simulated 
data based localization. It seems to happen due to difference of probability density function (PDF) of noise of received signals between simulated data and measured data.

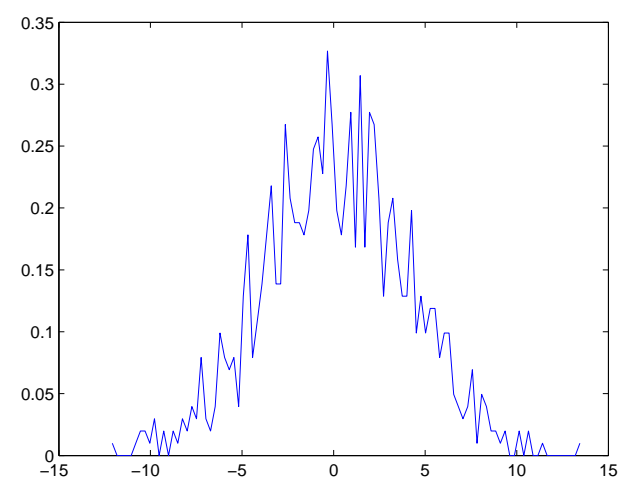

(a) PDF of noise of simulated data

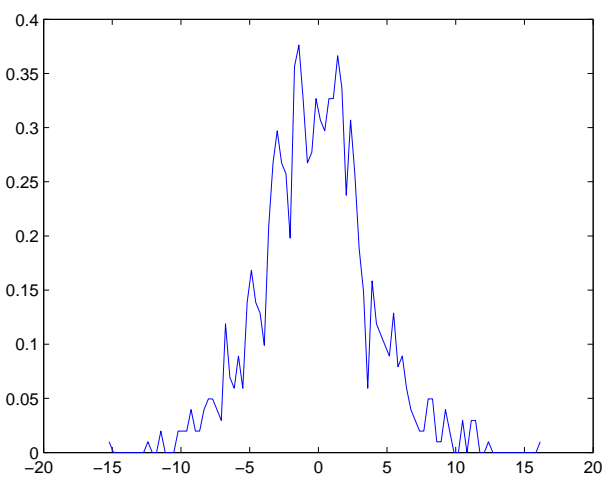

(b) PDF of noise of measured data

Figure 4.3: Comparison of PDF of noise for RSS

Therefore, the PDF of noise in simulated data and measured data are given to compare to each other as given in Figure 4.3. As a measure of peakedness kurtosis can be calculated, which is zero for normal distribution. If the kurtosis of certain distribution is larger than zero, one can say it is "peakier" than normal distribution. The kurtosis (peakedness) of noise in simulated data and measured data can be computed by Matlab command kurtosis. And the kurtosis of noise in simulated data and measured data is -0.0295 and 0.7632 respectively. In other words, noise is concentrated closer to zero in measured data than those in simulated data, which causes the probability of less noise. The center peak of noise PDF in measured data is "peakier" than that in simulated data, normal distribution. Such a distribution is called leptokurtic distribution or sometimes referred to super Gaussian distribution [41].

Therefore, due to the higher probability of having noises close to zero in measured data, it is expected that more precise distance estimation is made from measured data. And since $\log _{10} \tilde{d}=\log _{10} d+n$ as written in the Equation 4.2 the relationship between the distance calculated from received signal $\tilde{d}$ and reals distance $d$ can be expressed as

$$
\tilde{d}=d \cdot 10^{-\frac{n}{n_{p}}} \propto d \cdot 10^{-n}
$$

where $\mathrm{d}, n$, and $n_{p}$ denotes the true distance, the noise of received signal, and the path-loss exponent, respectively. Since path-loss exponent $n_{p}$ is close to fixed value $(3.92 \mathrm{~dB})$ according to measured value [37], it is considered not to affect the value of estimated distance. The only factor affecting the estimated distance $\tilde{d}$ is $10^{-n}$. Therefore, the higher the noise is, the longer the difference between the estimated distance and the true distance is.

Furthermore, since $10^{-n}$ is not additive but multiplicative factor as shown in the Equation 4.5, as the transmission distance gets longer, the difference between 
the estimated distance and the true distance also becomes longer, which reduces the accuracy of localization. Since the accuracy of location estimate depends on how close the distance calculated from received signal is to the real distance, the value of Gaussian noise $n$ can be said to be key factor affecting the accuracy of the localization especially for the non-iterative algorithm non-cooperative such as LSE or joint map estimation. In consequence, measured received signal data based localization has higher possibility to get smaller error than simulated data based localization since there exist more noises which are close to zero in measured data.

We will now observe the accuracy of scaled conjugate gradient, one of an iterative LSE method, based on measured data and simulated data. As mentioned in section 2.2.3, this algorithm localizes the object to be found by using not only a communication between an anchor node and an unknown node but also between unknown nodes for the purpose of improving the accuracy. The location of the object is iteratively updated until there is no change in the location.

To avoid the failure of convergence of local minimum close location from desired minimum, the location estimated from non-cooperative LSE, is chosen as an initial point to be updated iteratively in the scaled conjugate gradient method, which is used as cooperative LSE. As observed in Figure 4.2 the scaled conjugate gradient method is a lot more precise than LSE. In addition, even though the estimated location from non-cooperative is selected as an initial point for the scaled conjugate gradient, the accuracy and stability of the scaled conjugate gradient based on simulated data is as good as measured data based scaled conjugate gradient.

\subsubsection{Joint map estimation}

This subsection provides the results of non-cooperative and cooperative joint map estimation based on both measured data and simulated RSS (received signal strength) data in order to compare the performance of algorithms against one another. The joint map estimation implemented in Chapter 3 is non-cooperative method, which estimates the location of a single unknown sensor node one by one. However, in this section, since all the sensor nodes are reachable to one another, it is possible to estimate the location in cooperative way by putting all unknown nodes in the matrices to be estimated. This method is can be called as a cooperative joint map estimation since this method localizes the object in a way that estimates the location by involving the signal strength data between unknown nodes. In other word, this algorithm utilizes both the data between unknown node and anchor node and the data between unknown nodes. In this section, the localization result of cooperative joint map estimation is observed to see how different the non-cooperative and cooperative method are in terms of accuracy and stability.

As we can see from Figure 4.4, measured data based estimation has lower RMSE median than the simulated data based one in both non-cooperative and cooperative cases due to the presence of super Gaussian noise in measured RSS data as explained in previous subsection. In both measured and simulated RSS based localization results, it is easily seen that cooperative method has higher accuracy, 


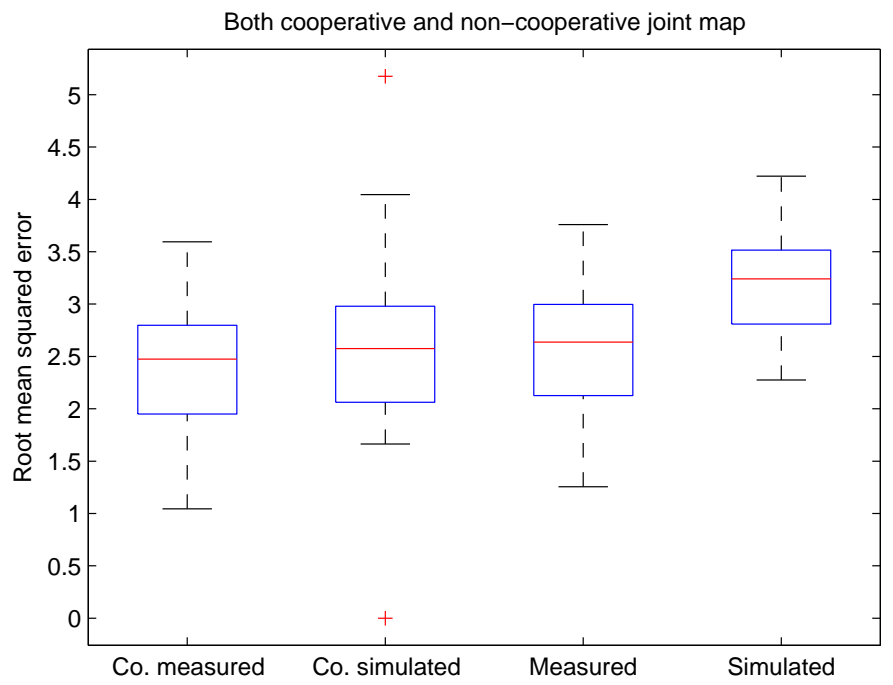

Figure 4.4: Joint map estimation based on RSS data

which means lower RMSE median.

Especially, in case of simulated data based localization, cooperative joint map method remarkably outperforms in aspect of accuracy. And in terms of stability, all four results seem comparable as the blue box of all results are about the height.

\subsubsection{Comparison of all algorithms}

This subsection observes results from both least squares estimation and joint map estimation to compare their performance.

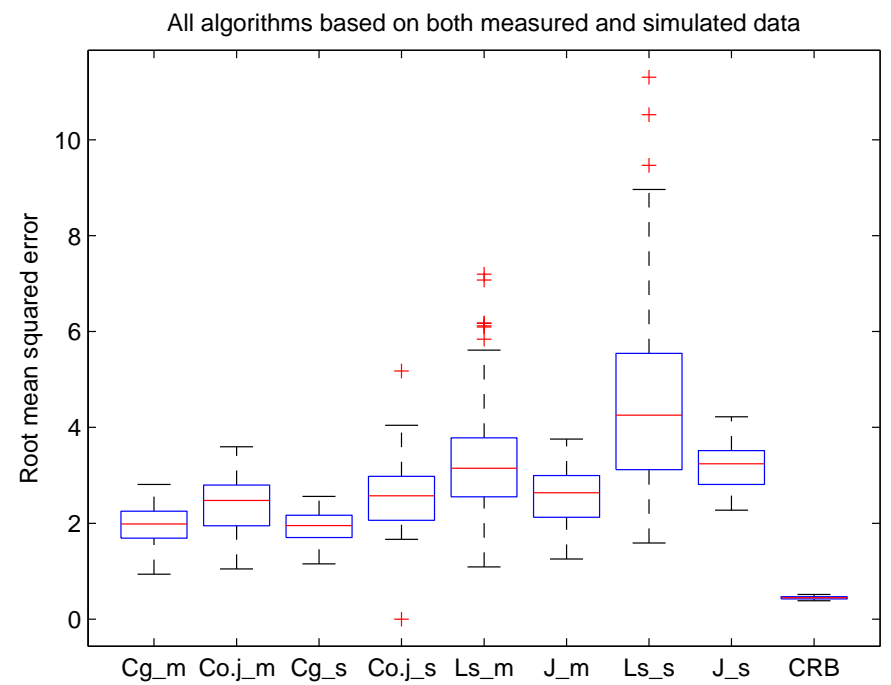

Figure 4.5: All algorithms with CRLB based on RSS data 
In Figure 4.5, the scaled conjugate gradient based on both measured data and simulated data, denoted as cg_m and cg_s respectively, has shown lower RMSE median than the cooperative joint map estimation based on both measured data and simulated data, denoted as co.j_m and co.j_s respectively. It proves that the scaled conjugate gradient has higher accuracy than cooperative joint map estimation. In addition, the stability of both measured and simulated data based scaled conjugate gradient estimation is better than cooperative joint map estimation.

However, when it comes to comparison of non-cooperative methods, the joint map estimation result based on both measured data and simulated data, denoted as J_m and J_s respectively, outperforms the non-cooperative LSE result, denoted as Ls_m and Ls_s respectively. This result implies that the space of the place where the campaign has been done is suitable enough for the joint map estimation to localize the object more accurately than non-cooperative LSE.

Non-cooperative LSE shows worse performance in both accuracy and stability aspects. In contrast, cooperative LSE, the conjugate gradient, has the best result in the accuracy and the stability aspects.

\subsection{Comparison for TOA based localization}

This section compares the localization results from TOA data measured in an experiment with that from simulated data to gage how reliable simulated data based localization is in real world. The same localization algorithms implemented in RSS based localization part are again used and the way of categorization on localization algorithms is also identical as RSS case. Table 4.4 shows fundamental parameter values of the measured data to give an intuition of how TOA simulated data is generated.

\begin{tabular}{|c|l|c|}
\hline \multicolumn{3}{|c|}{ Parameters and their values of TOA experiment } \\
\hline$\mu_{t}$ & Mean time delay error & $10.9 \mathrm{~ns}$ \\
\hline$v_{p}$ & Wave propagation speed (speed of light) & $3 \cdot 10^{8} \mathrm{~m} / \mathrm{s}$ \\
\hline$\sigma_{t}$ & Standard deviation & $6.1 \mathrm{~ns}$ \\
\hline
\end{tabular}

Table 4.4: The measurement data of TOA

Mean time delay is the average delay error occurred by attenuated, phase shifted, time-delayed, and attenuated multi-path replicas so-called multi-path error [37]. And the value of $\sigma_{t}$ has been calculated in a way that $\sigma_{d B}$ in RSS case is computed. In other words, $\sigma_{d B}$ is computed by averaging the difference between the measured arrival time and the arrival time without noise of all pairs out of 44 nodes as explained in Section 4.2.

An arrival time measured can be mathematically expressed as $\tau=\frac{d}{v_{p}}+\mu_{t}+n=$ $\frac{\tilde{d}}{v_{p}}+\mu_{t}$, where $\tilde{d}$ denotes the distance in noisy environment. Therefore, the distance in noisy environment $\tilde{d}$ is 


$$
\tilde{d}=v_{p} \cdot\left(\tau-\mu_{t}+n\right)
$$

Since the time of arrival $\mathrm{t}_{\mathrm{TOA}}$ depends only on noise which is additive and $v_{p}$ and $\mu_{t}$ is constant value, the relationship between the distance calculated from the arrival time and arrival time measured is linear.

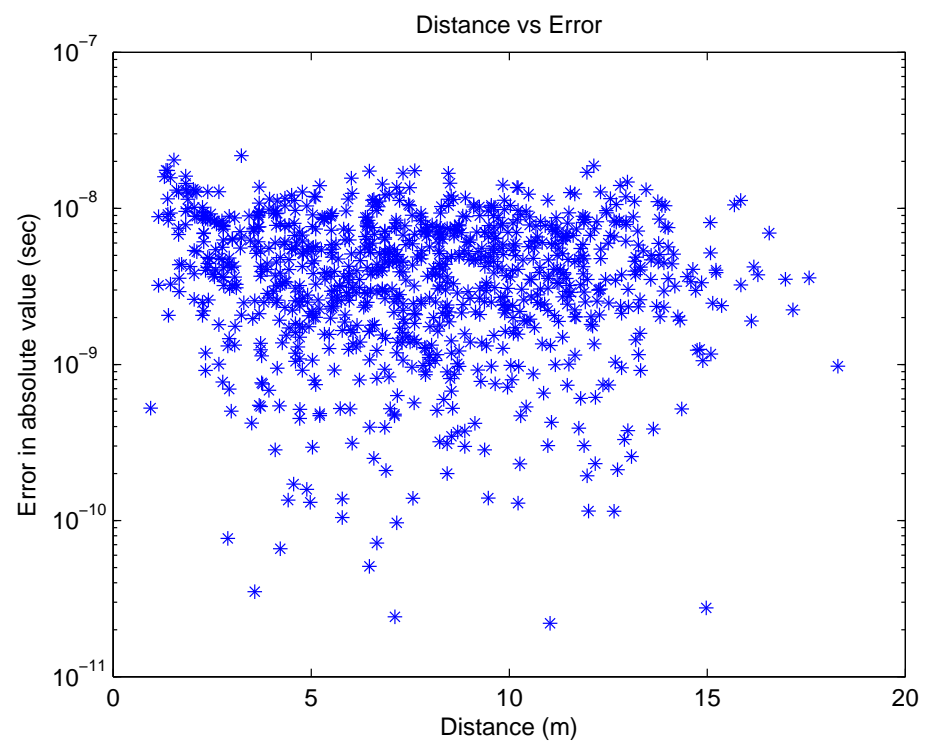

Figure 4.6: Distance vs Noise

Figure 4.6 shows absolute value of noise with vs distance in measured data. From this result, it can be observed that the noise presented during the experiment is not affected by a distance of a transmission.

The basic information regrading the set-up of a location estimate are as below.

\begin{tabular}{|l|c|}
\hline \multicolumn{1}{|c|}{ Parameter } & Value \\
\hline Number of unknown node & 10 \\
\hline Number of anchor node & 34 \\
\hline Times of node shift & 100 \\
\hline
\end{tabular}

Table 4.5: TOA based localization set-up

As it can be easily seen, it is exactly same as how the localization processed in RSS case. As done in RSS case, both non-cooperative and cooperative least squares estimation results based on both measured and simulated data are provided first, and joint map estimation results follow.

\subsubsection{Least squares based method}

The same localization algorithms and categorization as used in RSS case are applied in this TOA based localization part. A non-cooperative and a cooperative least squares estimation results are first given in this subsection as done in RSS case.

As shown in Figure 4.7, simulated data based localization result has lower root 


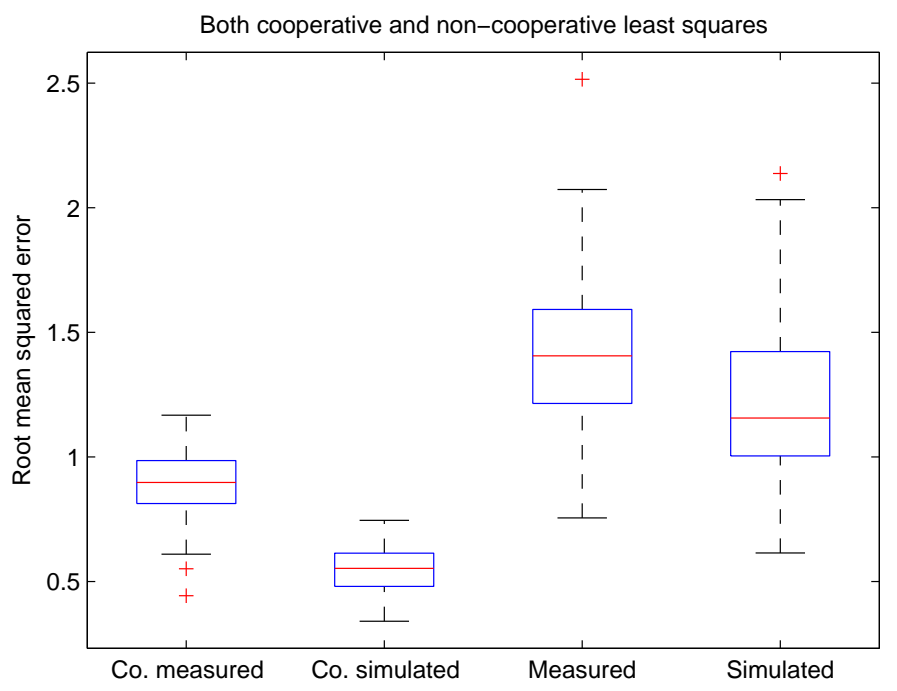

Figure 4.7: TOA based least squares estimation

mean squared error (RMSE) indicating higher accuracy than measured data based result in both non-cooperative and cooperative least squares estimation. What is most interesting about the result is that the cooperative method's result is influenced by non-cooperative algorithm. Cooperative LSE selects the location estimated by the non-cooperative LSE as an initial point to be updated iteratively and measured data based non-cooperative LSE result is worse than simulated data based one. Therefore, cooperative LSE based on measured data shows worse accuracy than same algorithm based on simulated data.

However, cooperative LSE result in RSS case was not affected by the result of non-cooperative LSE that much. Therefore, it can be concluded that the cooperative results are more intimately related to the non-cooperative results compared to RSS data based cooperative LSE. In terms of stability, both measured and simulated data based results using non-cooperative least squares estimation are similar so are both measured and simulated data based results using cooperative methods.

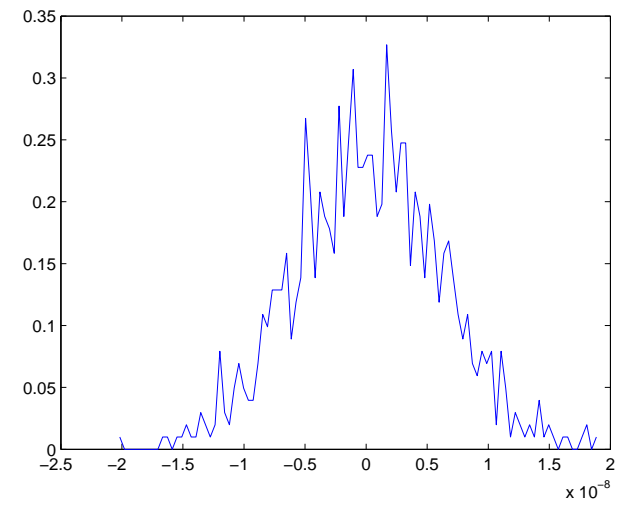

(a) PDF of noise of simulated data

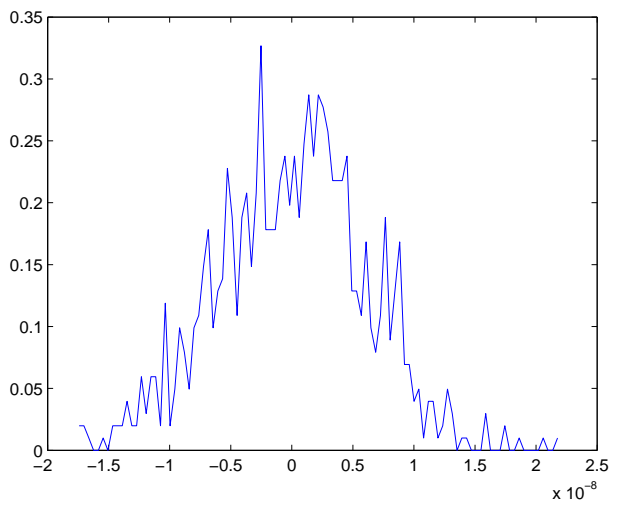

(b) PDF of noise of measured data

Figure 4.8: Comparison of PDF of noise for TOA 
Figure 4.8 is given to compare the noise of both measured signal strength data and simulated signal strength data as done previously. Although it is not very obvious, it looks like there are more noises deviated far from zero in simulated data than in measured data. And the kurtosis (peakedness) calculated of noise in simulated data and measured data was 0.1249 and 0.0665 respectively. In other words, noise distribution in simulated data is slightly "peakier".

\subsubsection{Joint map estimation}

This subsection shows the results of non-cooperative and cooperative joint map estimation based on both measured and simulated TOA data to observe the performance of all four algorithms.

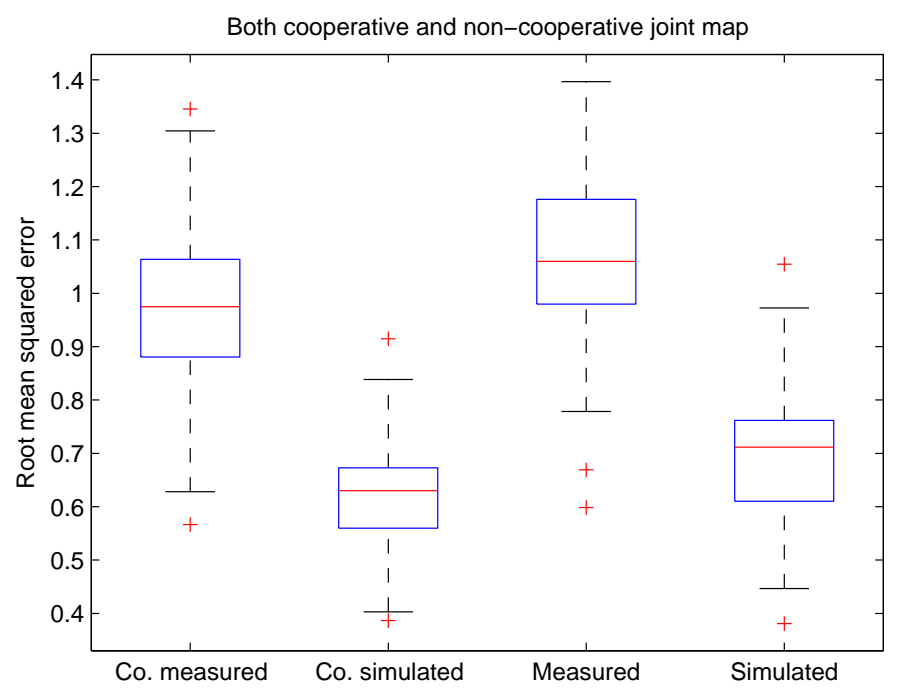

Figure 4.9: Joint map estimation based on TOA data

According the Figure 4.9, the difference of accuracy between the measured and simulated data based location estimate is longer compared to LSE case shown in previous subsection. Even non-cooperative estimate based on simulated data has better accuracy than cooperative localization result based on measured data. It can be said that joint map estimation is more sensitive with respect to noise distribution compared to LSE.

However, since the height of the blue box representing the range of values between $25 \%$ and $75 \%$ are about to same in all joint map's results, it is difficult to conclude that a noise affects a stability. As in RSS case, cooperative method's result proves its higher accuracy than non-cooperative localization in both measured data and simulated data based localization. 


\subsubsection{Comparison of all algorithms}

In this subsection, all results of localization are displayed and evaluated in order to give an idea which algorithm has better accuracy between LSE and joint map estimation.

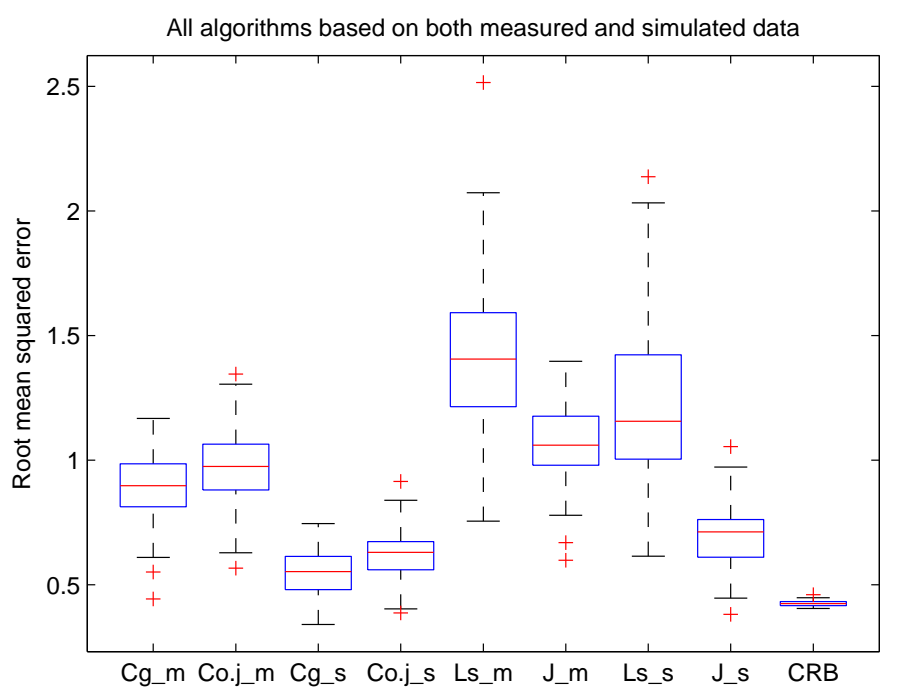

Figure 4.10: All algorithms with CRLB based on TOA data

Figure 4.10 shows that cooperative LSE (the scaled conjugate gradient) based on both measured data and simulated data, denoted as cg_m and cg_s respectively, has shown lower RMSE median than the cooperative joint map estimation based on both measured data and simulated data, denoted as co.j_m and co.j_s respectively. Therefore, the scaled conjugate gradient proves its higher accuracy than cooperative joint map estimation based on both TOA data and RSS data. Furthermore, in terms of the stability, LSE and joint map estimation are similar based on both measured data and simulated data.

However, the non-cooperative localization case joint map estimation, denoted as J_m and J_s respectively, outperforms the non-cooperative LSE result based on both measured data and simulated data, denoted as Ls_m and Ls_s respectively. As concluded in RSS part, the size of the place where the TOA measurement has been conducted is suitable enough for the joint map estimation to perform better than non-cooperative LSE in both accuracy and stability aspects.

Like the result in RSS case, non-cooperative LSE has the poorest result in both accuracy and stability. In contrast, cooperative LSE, the conjugate gradient, shows the best performance. 


\section{Chapter 5}

\section{Conclusions}

Throughout this thesis, a localization using wireless sensors has been explained theoretically and a simulation regarding the localization under certain scenario has been implemented. In the theoretical part, Chapter 1 and Chapter 2, the structure and the application of wireless sensor nodes has been introduced briefly. In addition, both non-cooperative and cooperative localization algorithms were introduced with equations when needed. The following two chapters, the experimental part, provided simulation results to observe the characteristic of each of localization algorithms described in the theoretical part.

In Chapter 3, the location estimate has been conducted in the scenario where anchor nodes are ployed in three different grid pattern (square, triangle, and random grid) in a squared space $(160 \mathrm{~m}$ by $160 \mathrm{~m})$. The localization results have been observed by varying a density first, a transmission range later. In Chapter 4, a localization result based on simulated data is compared to measured data based localization for both RSS and TOA case. In this chapter joint map estimation has been also implemented in cooperative manner, the way of localizing the unknown node using both anchor nodes and other unknown nodes located inside its transmission range to get better accuracy and to overcome transmission range limit problem, in order to compare with non-cooperative joint map estimation.

The simulation given in Chapter 3 proved that the accuracy of all estimation methods was improved as the anchor nodes are denser. Moreover, cooperative method were proven to be generally better than other non-cooperative method in aspect of accuracy. However, in mild condition- for example, highly dense environment or the environment where noise level is low- the joint map estimation showed very high accuracy even higher than the conjugate gradient for some cases as shown in Chapter 3. Cramér-Rao lower bound, which is the lower bound of unbiased estimator has been given to evaluate the performance of localization algorithms implemented.

In RSS case, the scaled conjugate gradient, the most accurate algorithm among all, yet still needs to be improved more to reach Cramér-Rao lower bound. In addition, the accuracy of all algorithms gets even worse as a transmission range is longer, which should be opposite according to [18]. Therefore, the algorithm suitable for non-linear relation between measurement and distance is needed to be implemented 
to get better result. However, in TOA case, the scaled conjugate gradient almost achieved Cramér-Rao lower bound.

It was observed that linear least squares estimation showed poor accuracy as the transmission range of a anchor node was longer in RSS based localization. That is caused by non-linear relationship between the signal strength and distance. And the accuracy of joint map estimation was drastically worse as the transmission range of a anchor node was longer in both RSS and TOA based localization. Therefore, the joint map estimation seemed to have poor accuracy when a transmitting distance is long regardless of type of noise. Interestingly in joint map estimation case, the localization with square grid pattern generally showed higher accuracy than other grid patterns in both RSS and TOA based localization.

In Chapter 4, the localization results based on measured data has slightly higher accuracy than simulated data based location estimate in RSS case. It was caused by super Gaussian noise distribution in measured received signal whereas noise present in simulated received signal was Gaussian noise. However, as the result proved simulated data based localization was quite close to measured data based one, meaning that the simulated data based localization result is reasonable.

And when it comes to a comparison of algorithms performance, scaled conjugate gradient performed the best. And cooperative joint map estimation outperformed the non-cooperative method although there was not much difference in the accuracy between two methods. In addition both non-cooperative and cooperative joint map estimation had higher accuracy than linear least squares estimation. This result was expected since the size of the place where the campaign was conducted is only much smaller than the place in the scenario of Chapter 3. Therefore, through simulated data and measured data based localization it is proven that the joint map estimation can be said to be more suitable algorithm than non-cooperative least squares algorithm in the small office environment.

However, the most significant result proven through Chapter 3 and Chapter 4 that is the cooperative localization outperforms non-cooperative one (the traditional method), which implies the way the future localization method should be. 


\section{Bibliography}

[1] Stefano Tennina, Fabio Graiosi, Fortunato Santucci, "Distributed and Cooperative Localization Algorithms for WSN in GPS-less Environments," [online]. Available from: http://www.cttc.es/resources/doc/081008-mdr-distributedand-cooperative-localization-algorithms-for-wsns-in-gps-less-environments45462.pdf

[2] D. Estrin, L.Girod, G. pottie, and M. srivastava, "Instrumenting the world with wireless sensor networks," in proc. IEEE Int. Conf. Acoustics, Speech, Signal Processing, vol. 4, pp. 2033-2036, Salt Lake City, Utah,USA, May 2001.

[3] B.R. Badrinath, M. Srivastava, K, "Special Issue on Smart and Environments," IEEE personal communications, Oct. 2000.

[4] "A Design and Implementation of Wireless Sensor Gateway for Efficient Querying and Managing through World Wide Web," IEEE Transactions on Consumer Electronics, vol. 49, no. 4, pp. 1090-1097, Nov. 2003.

[5] Feng Zhao, and Leonidas Guibas, WIRELESS SENSOR NETWORKS, MORGAN KAUFMANN PUBLISHER, 2004.

[6] E. Stavrou, wireless sensor networks pt1: introduction, [online]. Available from: http://webhosting.devshed.com/c/a/Web-Hosting-Articles/WirelessSensor-Networks-pt-1-Introduction/

[7] R. Rayudu, "Design Considerations for Wireless Sensor Nodes," UbiComp India 2008.

[8] Bo Zhao, Huazhong Yang, "Design of Radio-Frequency Transceivers for wireless Sensor Networks," InTech, [online]. Available from: http://www.intechopen.com/articles/show/title/design-of-radio-frequencytransceivers-for-wireless-sensor-networks

[9] Alessandro Aldini, Gilles Barthe, Roberto Gorrieri, Foundations of Secturity Analysis and Design V, Springer, 2009.

[10] BiPOM Electronics, Inc. "Microcontroller to Sensor Interfacing Techniques," [online]. Available from: http://www.bipom.com/documents/lectures/Microcontroller\%20to \%20Sensor\%20Interfacing\%20Techniques.pdf 
[11] Roberto Verdone, Davide Dardari, Gianluca Mazzini, Andrea Conti, Wireless Sensor and Actuator Networks, Academic Press, 2008.

[12] Randolph L. Moses, Dushyanth Krishnamurthy, and Robert M. Patterson,"A self-localization method for wireless sensor networks," EURASIP Journal on Applied Signal Processing, no. 4, pp. 348-358, Mar. 2003.

[13] A. R. Kulaib, R.M. Shubair, M. A. Al-Qutayri, Jason W. P. Ng, " An Overview of Localization Techniques for Wireless Sensor Networks," in International Conference on Innovations in information Technology, Apr. 2011, pp. 167-172.

[14] Breeze wireless communications Ltd., "Radio signal propagation," [online]. Available from:didier.quartier-rural.org/implic/ran/sat_wifi/sigprop.pdf

[15] K. Pahlavan, A.Levesque, Wireless Information Networks. Hoboken, NJ: Wiley, 1995.

[16] Alan J. Coulson, Allan G. Williamson, Rodney G. Vaughan, "A Statistical Basis for Lognormal Shadowing Effects in Multipath Fading Channels," IEEE TRANSACTIONS ON SIGNAL PROCESSING, vol. 46, no. 8, pp. 494-502, April 1998.

[17] Paolo Barsocchi, "Channel models for terrestrial wireless communications: A survey," IEEE Communications Surveys and Tutorials, Technical report, 2006. [Online]. Available from: http://puma.isti.cnr.it/dfdownload.php?ident=/cnr.isti/2006-TR-16

[18] Neal patwari, Joshua N. Ash, Spyros Kyperountas, Alfred O. Hero, Randolph L. Moses,Neiyer S. Correal, "Locating the Nodes: Cooperative localization in wireless sensor networks," IEEE SIGNAL PROCESSING MAGAZINE, vol. 52, no. 2,pp. 54-69, July 2005.

[19] Ralf Lehnert, ed. Energy-Aware Communications, Springer, 2011.

[20] G.D. Durgin, Space-Time Wireless Channels, Englewood Cliffs, NJ: Prentice Hall, 2002.

[21] T. Karalar, J. Rabaey, "An RF ToF Based Ranging Implementation for Sensor Networks," IEEE International Communications Conference, June 11-15 2006, Istanbul, Turkey.

[22] G. Clifford Carter, "Coherence and time delay estimation," Proc. of the IEEE, vol. 75, no. 2, pp. 236-255, Feb. 1987.

[23] Charles H. Knapp, G. Clifford Carter, "The generalized correlation method for estimation of time delay," IEEE transaction acoustics, speech, and signal proc., vol. 24, no. 4, pp. 320-327, August 1976. 
[24] E.R. Robinson, A. H. Quazi, "Effect of sound-speed profile on differential time delay estimation," J. Acous. Soc. America, vol. 77, no. 3, pp. 1086-1090, March 1985 .

[25] A. H. Quazi, "An overview on the time delay estimate in active and passive systems for target localization," IEEE transcations of Acous. Speech signal proc., vol. ASSP-29, no. 3, pp. 527-533, June 1981.

[26] B.B. Peterson, C. Kmiecik, R. Hartnett, P.M. Thompson, J. Mendoza, and H. Nguyen, "Spread spectrum indoor geolocation," J. Inst. Navigat., vol. 45, no. 2, pp. 97-102, 1998.

[27] Tufan C. Karalar, Jan Babaey, "An RF ToF Based Randing Implementation for Sensor Networks," in Communications, 2006. ICC '06. IEEE International Conference on, vol. 7, pp. 3347-3352, June 2006.

[28] David C. Lay, Linear Algebra and its applications, Addison Wesley, 2002.

[29] Silke Feldmann, Kyandoghere Kyamakya, Ana Zapater, Zighuo Lue, "An indoor Bluetooth-based positioning system: concept, Implementation and experimental evaluation," presented at ICWN"03, Las Vegas, USA, 2003.

[30] Andreas Richter, "Joint map estimation and localization using distance measurements to landmarks with unknown location,"on Signals, Systems and Computers (ASILOMAR), 2010 Conference Record of the Forty Fourth Asilomar Conference, November 2010.

[31] Dapeng Qiao, Grantham K.H. Pang, "Localization in Wireless Sensor Networks with Gradient Descent,"on Communications, Computers and Signal Processing (PacRim), 2011 IEEE Pacific Rim Conference, August 2011.

[32] Hervé Abdi, The SAGE Encyclopedia of Social Science Research Methods, Vol.1, Alan E. Bryman, Alan Bryman, Tim F. Liao, editor. C.A: Thousand Oaks, pp.559-561, 2003

[33] Trond Hjorteland, June 1999, THE ACTION VARIATIONAL PRINCIPLE IN COMOLOGY [Ph.D. thesis]. the University of Oslo, Oslo.

[34] Andreas Zell, Niels Mache, Ralf Hübner, Günter Mamier, Michael Vogt, Michael Schmalzl, Kai-Uwe Herrmann, SNNS (STUTTGART NEURAL NETWORK SIMULATOR), Kluwer Academic Publishers, 1994.

[35] Martin Møller, A Scaled Conjugate Gradient Algorithm for Fast Supervised Learning, Neural Networks, vol. 6, pp. 525-533, June 1993

[36] S.T. Smith, "Intrinsic cramer-rao bounds and subpace estimation accuracy," The first IEEE Sensor Array and Multichannel workshop, pp.489-pp.493, March 2000. 
[37] Neal Patwari, Alfred O. Hero, Matt Perkins, Neiyer S. Correal, and Robert J. O'Dea, "Relative Location Estimation in Wireless Snesor Networks," IEEE TRANSACTIONS ON SIGNAL PROCESSING, vol. 51, no. 8, pp. 2137-2148, August 2003.

[38] Kegen Yu, Y. Jay Guo, "Improving Anchor Position Accuracy for 3-D localization in Wireless Sensor Networks,"on Communications, 2008. ICC '08. IEEE Internationalization Conference, pp. 951-955, May 2008.

[39] The Royal Academy of Engineering, The Study of Root Mean Square (RMS) Value [online]. Available from: http://www.raeng.org.uk/education/diploma/maths/pdf/ exemplars_engineering/8_RMS.pdf

[40] Neal Patwari, Wireless Sensor Network Localization Measurement Repository [online]. Available from: http://web.eecs.umich.edu/ hero/localize/

[41] Khurram Waheed, Fathi M. Salam, "Blind source recovery using an adaptive generalized Gaussian score function," in Proc. 45th Midwest Symposium Circuits Systems (MWSCAS), vol. 2, pp. II - 418 - pp. II -421, Aug. 2002. 